Discussion Paper No. 09-084

Methodologische Grundlagen einer Evaluation von Wettbewerbspolitik

Kai Hüschelrath

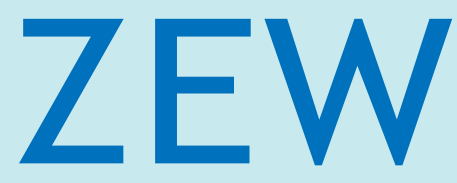

Zentrum für Europäische Wirtschaftsforschung $\mathrm{GmbH}$

Centre for European

Economic Research 
Discussion Paper No. 09-084

\title{
Methodologische Grundlagen einer Evaluation von Wettbewerbspolitik
}

\author{
Kai Hüschelrath
}

Download this ZEW Discussion Paper from our ftp server:

ftp://ftp.zew.de/pub/zew-docs/dp/dp09084.pdf

Die Discussion Papers dienen einer möglichst schnellen Verbreitung von neueren Forschungsarbeiten des ZEW. Die Beiträge liegen in alleiniger Verantwortung der Autoren und stellen nicht notwendigerweise die Meinung des ZEW dar.

Discussion Papers are intended to make results of ZEW research promptly available to other economists in order to encourage discussion and suggestions for revisions. The authors are solely responsible for the contents which do not necessarily represent the opinion of the ZEW. 


\section{Das Wichtigste in Kürze}

Im Jahre 2005 fand im Rahmen eines OECD Policy Roundtables ein intensiver Meinungsaustausch der Mitgliedsstaaten zu dem Thema ,Evaluation of the Actions and Resources of the Competition Authorities’ statt. Eine wesentliche Erkenntnis der Gespräche bestand in der Feststellung, dass „[c]onsiderable work remains to be done to refine the methodologies used to evaluate the effectiveness of completed competition policy interventions“ (OECD, 2005,. S. 10). Obwohl seit der Formulierung dieser Arbeitsvorgabe erst einige Jahre vergangen sind, so hat die Bedeutung der Evaluation von Wettbewerbspolitik seitdem deutlich zugenommen - sowohl im wissenschaftlichen Bereich als auch im Bereich der Umsetzung in der Praxis. Die Schwerpunkte der Arbeiten lagen dabei allerdings nicht so sehr auf einer methodologischen Strukturierung und Aufbereitung der gesamten Problemstellung, sondern eher in der Verfeinerung bzw. Überprüfung bestimmter Methoden, der Betrachtung ausgewählter Fallstudien sowie der Umsetzung staatlicher Vorgaben der Qualitätskontrolle bzw. Rechenschaftspflichten.

Vor diesem Hintergrund verfolgt dieser Beitrag nicht nur das Ziel einer Identifikation und Charakterisierung genereller methodologischer Bausteine einer Politikevaluation, sondern er beabsichtigt vor allem eine Einordnung der Erkenntnisse bestehender wettbewerbspolitischer Arbeiten in den geschaffenen methodologischen Rahmen vorzunehmen. Diese Vorgehensweise erlaubt nicht nur eine systematische Darstellung der methodologischen Überlegungen bestehender Studien, sondern ermöglicht gleichzeitig eine Identifikation von Problemen bei der Evaluation von Wettbewerbspolitik und somit die Ableitung von Forschungsbedarf. 


\title{
METHODOLOGISCHE GRUNDLAGEN EINER EVALUATION VON WETTBEWERBSPOLITIK
}

\author{
Kai Hüschelrath*
}

Im Jahre 2005 fand im Rahmen eines OECD Policy Roundtables ein intensiver Meinungsaustausch der Mitgliedsstaaten zu dem Thema ,Evaluation of the Actions and Resources of the Competition Authorities' statt. Eine wesentliche Erkenntnis der Gespräche bestand in der Feststellung, dass „[c] [considerable work remains to be done to refine the methodologies used to evaluate the effectiveness of completed competition policy interventions" (OECD, 2005,. S. 10). Obwohl seit der Formulierung dieser Arbeitsvorgabe erst einige Jahre vergangen sind, so hat die Bedeutung der Evaluation von Wettbewerbspolitik seitdem deutlich zugenommen - sowohl im wissenschaftlichen Bereich als auch im Bereich der Umsetzung in der Praxis. Die Schwerpunkte der Arbeiten lagen dabei allerdings nicht so sehr auf einer methodologischen Strukturierung und Aufbereitung der gesamten Problemstellung, sondern eher in der Verfeinerung bzw. Überprüfung bestimmter Methoden, der Betrachtung ausgewählter Fallstudien sowie der Umsetzung staatlicher Vorgaben der Qualitätskontrolle bzw. Rechenschaftspflichten. Vor diesem Hintergrund verfolgt dieser Beitrag nicht nur das Ziel einer Identifikation und Charakterisierung genereller methodologischer Bausteine einer Politikevaluation, sondern er beabsichtigt vor allem eine Einordnung der Erkenntnisse bestehender wettbewerbspolitischer Arbeiten in den geschaffenen methodologischen Rahmen vorzunehmen. Diese Vorgehensweise erlaubt nicht nur eine systematische Darstellung der methodologischen Überlegungen bestehender Studien, sondern ermöglicht gleichzeitig eine Identifikation von Problemen bei der Evaluation von Wettbewerbspolitik und somit die Ableitung von Forschungsbedarf.

KEYWORDS: Wettbewerbspolitik, Evaluation, Kartelle, Horizontale Fusionen

JEL: K21, L40

\footnotetext{
* Dr. Kai Hüschelrath ist Wissenschaftlicher Mitarbeiter am Zentrum für Europäische Wirtschaftsforschung (ZEW) in Mannheim sowie Juniorprofessor für Industrieökonomik und Wettbewerbsstrategie an der WHU Otto Beisheim School of Management in Vallendar. E-mail: hueschelrath@zew.de Ich danke Christian Ewald (Bundeskartellamt) und Nina Leheyda für sehr hilfreiche Diskussionen in der konzeptionellen Phase dieses Beitrags sowie den Teilnehmern an einem Antitrust Law and Economics Workshop in Tübingen im Juni 2009 für hilfreiche Kommentare. Etwaige Fehler oder Ungenauigkeiten gehen selbstverständlich allein zu meinen Lasten.
} 
"Encouraging government bodies to conduct performance evaluations is not only good public policy, it is likely to be a key ingredient of future attempts by competition authorities to demonstrate the value of competition law to broader audiences. ... An a priori presumption of efficacy is a weak substitute for a systematic assessment of outcomes."

William Kovacic (2006, S. 505)

\section{Einführung}

In den letzten Jahren sind in verschiedenen Ländern verstärkte Bemühungen beobachtbar, wettbewerbspolitische Aktivitäten einer Evaluation zu unterziehen. So nimmt beispielsweise das Office of Fair Trading (2008a) in Großbritannien - vor dem Hintergrund einer entsprechenden Vorgabe seitens des Finanzministeriums - seit einigen Jahren eine Quantifizierung des Nutzens seiner Aktivitäten für die Verbraucher vor und stellt diesen dem bereitgestellten Budget gegenüber. Die niederländische Wettbewerbsbehörde (NMa) ist vor kurzem sogar dazu übergegangen, die positiven Auswirkungen ihrer Aktivitäten auf makroökonomische Faktoren wie Wachstum und Beschäftigung zu messen (vgl. dazu Don et al., 2008). ${ }^{1}$ Im Vergleich zu diesen beiden relativ neuen Evaluationsansätzen müssen die Vereinigten Staaten als routiniert im Bereich der Evaluation von Wettbewerbspolitik bezeichnet werden, da dort bereits seit 1993 der so genannte Government Performance and Results Act (GPRA) vorschreibt, die ungefähren Auswirkungen der Aktivitäten der beiden Wettbewerbsbehörden mit Hilfe geeigneter Leistungsindikatoren zu dokumentieren (vgl. dazu Werden, 2008).

In Ergänzung zu den bislang erwähnten ganzheitlichen Untersuchungen zu den Wohlfahrtswirkungen wettbewerbspolitischer Aktivitäten sind ferner auch Studien zu finden, deren Ziel es im Wesentlichen ist, bestimmte interne Prozesse von Wettbewerbsbehörden zu evaluieren und in der Folge gegebenenfalls zu verbessern. Als Beispiele hierfür können eine Studie zur Wirksamkeit von Fusionsauflagen der Europäischen Kommission (2005) sowie eine vor kurzem vorgelegte Studie mit vergleichbarer Ausrichtung der zweiten britischen Wettbewerbsbehörde - der Competition Commission - angesehen werden (vgl. Competition Commission, 2008).

Komplementär zu den gerade skizzierten behördeninternen Studien ist auch auf akademischer Ebene in den letzten Jahren ein zunehmendes Interesse für die Evaluation von Wettbewerbspolitik feststellbar. So finden sich hier zwar eher keine vollständigen Evaluationen von Wettbewerbspolitiken oder -gesetzen, dafür aber eine Reihe von Studien, die beispielsweise die Effektivität der wettbewerbsbehördlichen Fusionskontrolle untersuchen (vgl. beispielsweise Duso et al., 2006), die Wirkungen wettbewerbspolitischer Eingriffe quantifizieren (vgl. beispielsweise Hüschelrath, 2009b) oder aber die Robustheit bestimmter Untersuchungsmethoden untersuchen (vgl. beispielsweise Weinberg und Hosken, 2009).

Vor diesem Hintergrund verfolgt dieser Beitrag nicht nur das Ziel einer Identifikation und Charakterisierung genereller methodologischer Bausteine einer Politikevaluation, sondern er beabsichtigt vor allem eine Einordnung der Erkenntnisse bestehender wettbewerbspolitischer Arbeiten in den geschaffenen methodologischen Rahmen vorzunehmen. Diese Vorgehensweise erlaubt nicht nur eine systematische Darstellung der methodologischen Überlegungen bestehender Studien, sondern ermöglicht gleichzeitig eine Identifikation von Problemen bei der Evaluation von Wettbewerbspolitik und somit die Ableitung von Forschungsbedarf.

\footnotetext{
${ }^{1}$ Sogar die Competition Regulatory Authority der sich in Kronbesitz befindlichen Kanalinsel Jersey - mit rund 90.000 Einwohnern - publizierte im vergangenen Jahr eine Kurzstudie zum Thema ,Impacts of Competition Policy in the Bailiwick of Jersey’ (vgl. Jersey Competition Regulatory Authority, 2008).
} 


\section{Grundlegende Bausteine einer Methodologie zur Politikevaluation}

Dem ursprünglichen Wortsinn folgend hat Evaluation mit Werten und Bewerten zu tun. Evaluationen sind daher ein omnipräsentes Phänomen welchem keinesfalls nur im volkswirtschaftlichen Kontext eine große Bedeutung zukommt, sondern beispielsweise ebenso im Bildungs-, Medien- oder Umweltbereich. ${ }^{2}$ Die Deutsche Gesellschaft für Evaluation e.V. (DeGEval) versteht unter Evaluation die "systematische Untersuchung des Nutzens oder Wertes eines Gegenstandes" (DeGEval, 2002: 13). Der Begriff ,Gegenstand' (bzw. auch ,Objekt') ist dabei weit gefasst und beinhaltet z.B. Programme, Projekte, Produkte, Maßnahmen, Leistungen, Organisationen, Politiken oder Technologien.

Wie bereits der Titel dieses Beitrags vermuten lässt, soll es im Weiteren jedoch nicht um jedwede Art der Evaluation gehen, sondern um die spezifische Form der Politikevaluation, also einer Bewertung staatlichen Handelns. Im Rahmen einer solchen Spezifizierung dienen Evaluationen allgemein der Ermittlung der Wirksamkeit und Wirtschaftlichkeit staatlichen Handelns. „Sie sollen dazu beitragen, die Erarbeitung neuer Maßnahmen (Gesetze, Verordnungen) auf sicherere Grundlagen abzustützen, deren Vollzug besser auf die Ziele auszurichten und generell die Transparenz über staatliches Handeln zu verbessern“ (Bundesamt für Justiz, 1999).

Im Rahmen einer Politikevaluation werden oftmals drei Hauptbestandteile unterschieden: der Gegenstand der Evaluation, die Wahl der Evaluationsmethodik (z.B. Peer Review, Fallstudien, Kosten-Nutzen-Analysen, Quantitative Methoden) sowie der Zeitpunkt der Evaluation (ex-ante, begleitend oder ex-post). Eine standardisierte Evaluationsmethodologie existiert dabei nicht und wird auch nicht angestrebt. In Abhängigkeit des genauen Evaluationsgegenstandes, des gewünschten Evaluationszeitpunkts sowie der Funktionen, der Ziele und des Kontexts der Evaluation sind diejenigen Kriteriensets, Indikatoren und Methoden auszuwählen und zu kombinieren, mit denen die aussagekräftigsten Ergebnisse (im Rahmen des vorgegebenen Budgets und der zur Verfügung stehenden Daten) erzielt werden können.

Obwohl ein festgelegtes Evaluationskorsett angesichts variabler Zielsetzungen vor unterschiedlichen institutionellen Hintergründen nicht erstrebsam scheint, so ist es dennoch von Nutzen, grundlegende Bausteine einer Politikevaluation zu definieren, welche dann im Rahmen einer spezifischen Evaluationsaufgabe den jeweiligen Gegebenheiten angepasst werden können. Abbildung 1 versucht dies unter teilweisem Einbezug der umfangreichen konzeptionellen Beiträge von Bussmann et al. (1997) und Stockmann (2000) zur Politikevaluation.

\footnotetext{
${ }^{2}$ Ein interessanter Überblick der unterschiedlichen Definitionen und Interpretationen des Begriffs Evaluation ist auf der folgenden Seite verfügbar: http://www.evaluieren.de/evaluat.ion/definiti.htm\#bildung .
} 


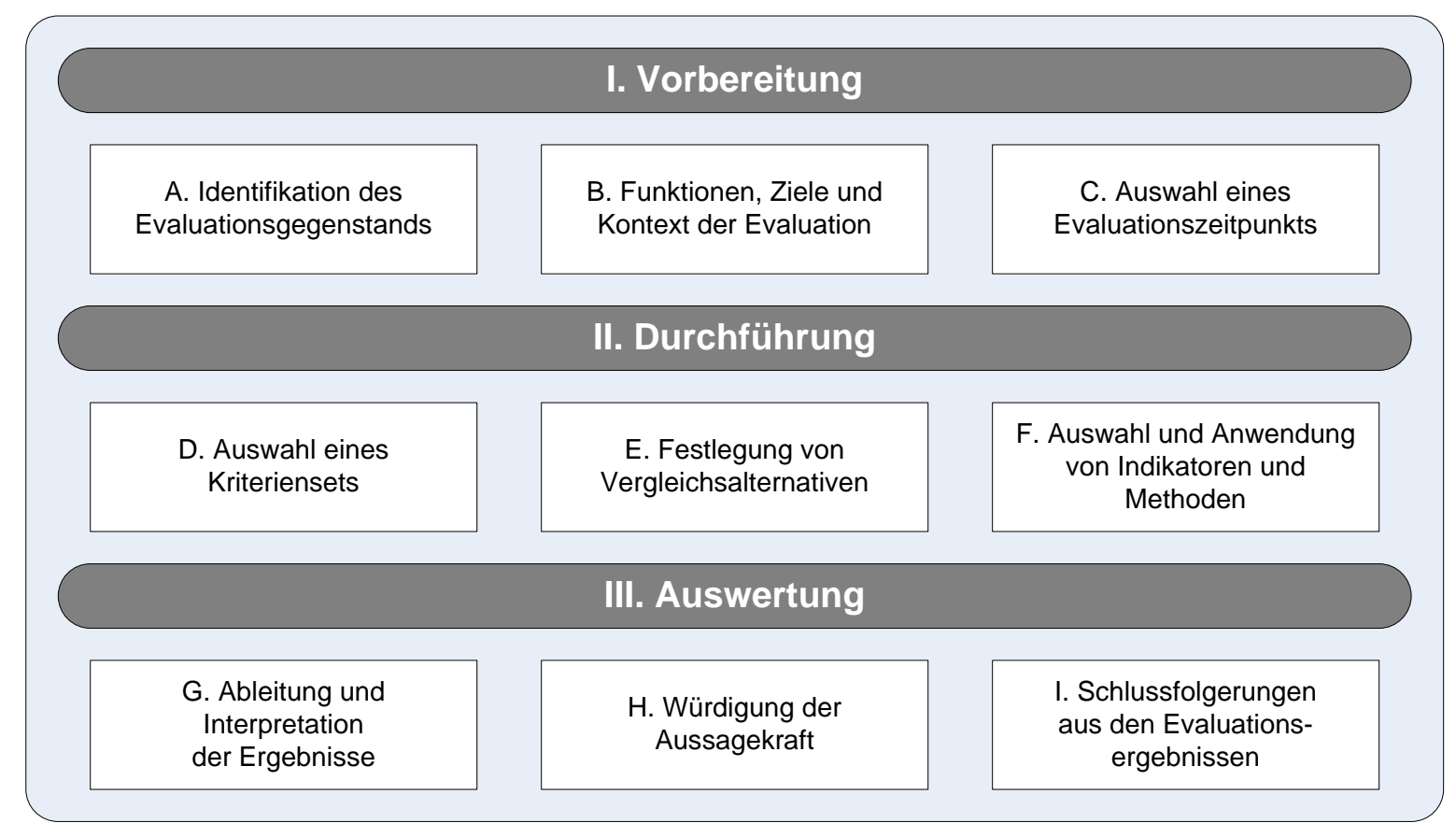

\section{Abbildung 1: Bausteine einer Methodologie zur Politikevaluation}

Wie Abbildung 1 verdeutlicht, lassen sich die unterschiedlichen Bausteine einer Methodologie zur Politikevaluation in drei Stufen aufteilen: Vorbereitung, Durchführung und Auswertung. Der Bereich der Vorbereitung beschäftigt sich grob formuliert mit der Identifikation des Evaluationsgegenstandes sowie der Forschungs- bzw. Untersuchungsfrage (Was?), den Funktionen, den Zielen und dem Kontext der Evaluation (Warum?) sowie dem Zeitpunkt der Evaluation (Wann?). Die sich anschließende Durchführungsstufe (Wie?) beginnt mit der Auswahl des Kriteriensets, gefolgt von der Festlegung von Vergleichsalternativen und der Auswahl und Anwendung der Indikatoren und Methoden. Die dritte Stufe der Methodologie zur Politikevaluation besteht wiederum aus drei Bausteinen, nämlich der Ableitung und Interpretation der Ergebnisse, der Würdigung der Aussagekraft sowie der Ableitung von Schlussfolgerungen aus den Evaluationsergebnissen. Um Wiederholungen zu vermeiden, erfolgt in den folgenden Abschnitten nur eine knappe Erläuterung der einzelnen Bausteine. Im anschließenden dritten Kapitel erfolgt dann eine genauere Betrachtung im Rahmen der Einordnung bestehender Arbeiten im Bereich der Evaluation von Wettbewerbspolitik. Generell gilt, dass im Folgenden lediglich Fragen der strategischen Planung und Ausrichtung von Evaluationen behandelt werden und demnach von operationalen Fragestellungen weitgehend abstrahiert wird (vgl. dazu detailliert Bussmann, 1995, sowie Kovacic, 2006, S. 533ff. für den spezifischen Bereich der Evaluation von Wettbewerbspolitik).

\subsection{Gegenstand, Ziele und Zeitpunkt}

Die Vorbereitungsstufe einer Politikevaluation besteht in der oben vorgeschlagenen Methodologie aus drei zentralen Bausteinen: Der Identifikation des Evaluationsgegenstands, der Charakterisierung der Funktionen, der Ziele sowie des Kontexts der Evaluation sowie der Auswahl des Zeitpunkts der Evaluation. Diese Bausteine werden im Folgenden kurz erläutert. Bereits an dieser Stelle sei darauf hingewiesen, dass die drei Bausteine im Rahmen der Vorbereitungsstufe nicht zwangsläufig iterativ abgearbeitet werden müssen, sondern dass durchaus eine simultane Betrachtung möglich ist und auch sinnvoll sein kann. 


\section{Identifikation des Evaluationsgegenstands}

Wie bereits in einem der vorangegangenen Abschnitt angedeutet, wird der Begriff des Evaluationsgegenstands grundsätzlich sehr weit gefasst und beinhaltet z.B. Programme, Projekte, Produkte, Maßnahmen, Leistungen, Organisationen, Politik oder Technologien. Aus diesem Grund ist es zu Beginn des Projekts von großer Bedeutung, den Gegenstand der Evaluation so klar und genau wie möglich festzulegen. Aufgrund der oftmals vorliegenden Vielfalt und Komplexität der zu berücksichtigenden Zusammenhänge bietet sich im Regelfall eine Begrenzung der Evaluation auf spezifische Teilgegenstände (z.B. Projekte oder Maßnahmen) oder spezifische Wirkungsaspekte von staatlichem Handeln an (vgl. dazu Bussmann et al., 1997, S. 66). In diesem Zusammenhang ist es auch notwendig, eine möglichst konkrete Forschungs- bzw. Untersuchungsfrage zu definieren, auf die die Evaluation ausgerichtet werden soll (vgl. Bussmann, 1995, S. 15f.). Unter Umständen ist es in diesem Zusammenhang auch bereits sinnvoll, das Zielsystem des zu evaluierenden Gegenstands zu benennen, welches dann im Folgenden auf den Zielerreichungsgrad hin überprüft werden soll. Im Rahmen der hier entwickelten Methodologie wird diese Aktivität allerdings der Durchführungsstufe zugeordnet.

\section{Funktionen, Ziele und Kontext der Evaluation}

Im Rahmen der Vorbereitung einer Evaluation sollten eine Reihe von Fragen beantwortet werden, um dann im sich anschließenden Durchführungsteil geeignete Bewertungskriterien und Untersuchungsmethoden identifizieren zu können und schließlich aussagekräftige Ergebnisse zu erhalten. Neben den bereits gerade separat angesprochenen zentralen Fragen nach dem Evaluationsobjekt und der Untersuchungsfrage zählen zu solchen einleitend zu klärenden Fragen beispielsweise die Identifikation der Beteiligten (insbesondere der Evaluierenden ${ }^{3}$ ), die Darstellung der Ursache, die Festlegung des bzw. der Ziele der Evaluation sowie die Charakterisierung des Kontexts in dem die Evaluation stattfindet. Auch der Zeitpunkt der Evaluation gehört grundsätzlich zu diesen vorbereitend festzulegenden Aspekten; dieser soll hier aber aus inhaltlichen Gründen getrennt als dritter Baustein charakterisiert werden.

Evaluationen können grundsätzlich eine oder mehrere Funktionen verfolgen. Stockmann (2000) unterscheidet grundsätzlich vier Funktionen. Die Erkenntnisfunktion hat ihren Fokus in der Sammlung und Bewertung entscheidungsrelevanter Daten über beispielsweise den Verlauf und die Ergebnisse des Evaluationsgegenstands, Bedürfnisse der Zielgruppe oder die Veränderung von Rahmenbedingungen. Demgegenüber steht im Mittelpunkt der Kontrollfunktion die Beobachtung einer Politik bzw. Maßnahme mit dem Ziel, Defizite zu erkennen um möglichst rasch steuernd eingreifen zu können. Gleichzeitig werden oftmals aber auch Informationen darüber generiert, ob alle Beteiligten ihren Verpflichtungen nachkommen. Die Dialogfunktion verfolgt den Zweck, das im Rahmen der Evaluation generierte Wissen in den Dialog mit den jeweiligen Stakeholdern (z.B. Mittelgeber, Durchführungsorganisation, Zielgruppen, sonstige Beteiligte und Betroffene einer Politik oder Maß-

\footnotetext{
${ }^{3}$ Evaluationen können entweder rein intern oder rein extern durchgeführt werden, es sind aber auch Mischungen (zwischen verschiedenen Teilprojekten) innerhalb größerer Evaluationen denkbar. Die Auswahl der jeweils am geeignetesten anzusehenden Vorgehensweise hängt von den Zielsetzungen ab. So können interne Evaluationen dabei helfen, Planung und Steuerung zu verbessern oder sie können der Rechenschaftslegung bei einer beaufsichtigenden Institution dienen. Sie können aber auch Forschungs- und Selbstverbesserungszielen folgen. Externe Evaluationen haben im Vergleich dazu oft die Aufgabe der Qualitätssicherung oder der Überprüfung der Einhaltung von Standards oder auch des länderübergreifenden Vergleichs verbunden mit einer Rückmeldung der relativen Performance. Externe Evaluationen haben generell das größere Potential, unabhängig und frei von ,Betriebsblindheit' zu sein. Ferner haben sie den zentralen Vorteil, dass auf das Methodenwissen von Experten zurückgegriffen werden kann, das intern nicht immer vorhanden sein muss. Auf der anderen Seite haben sie den zentralen Nachteil, dass die externen Evaluierenden oftmals nicht hinreichend mit den internen Gegebenheiten vertraut sind, um eine ausgewogene Evaluation in vollem Ausmaß sicherstellen zu können. Dies kann dann unter anderem Akzeptanzprobleme zur Folge haben.
} 
nahme) einfließen lassen zu können. Die Legitimitätsfunktion schließlich hat ihren Fokus in der Nutzung des generierten Wissens zur Dokumentation, mit welchem Input, welcher Output und welche Wirkungen erzielt wurden. Im Rahmen von ex-post Evaluationen lässt sich zudem die Nachhaltigkeit und der Wirkungsgrad der Politiken bzw. Maßnahmen belegen.

In direktem Zusammenhang mit den Funktionen von Evaluationen sind die grundsätzlichen Ziele von Evaluationen zu sehen. Eine zutreffende einführende Definition liefern Suhling und Ansorge (2008, S. 1): „Das Ziel einer Evaluation ist die Beurteilung der Konzeption, der Umsetzung und der Ergebnisse einer Maßnahme, um eine Rückmeldung über deren Wirksamkeit (den Zielerreichungsgrad) und ggf. auch deren Effizienz (die KostenNutzen-Bilanz) zu bekommen.“ Diese Definition deutet bereits an, dass Evaluationen durchaus verschiedene Ziele verfolgen können; in den meisten Fällen ist aber wohl eine Wirksamkeitsanalyse obligatorischer Bestandteil. Diese vergleicht die der Politik bzw. der Maßnahme zugrunde liegenden Ziele mit dem tatsächlich Erreichten. Die möglichen Zielsetzungen von Evaluationen sind damit aber noch nicht erschöpfend beschrieben. Bussmann et al. (1997, S. 45ff.) merken beispielsweise an, dass im Rahmen von Evaluationen nicht ausschließlich nach der Wirksamkeit der Maßnahmen gefragt werden muss, sondern durchaus auch nach der Wirtschaftlichkeit. Solche Wirtschaftlichkeitsanalysen setzen die Wirkungen eines Programms in Beziehung zu den eingesetzten Mitteln und Ressourcen (Kosten-Nutzen Analyse). Weiterhin nennen Bussmann et al. (1997) noch die Möglichkeit einer so genannten Prozessevaluation, die sich beispielsweise vor allem auf Fragen der Implementierung konzentriert. Beim so genannten Programm-Monitoring handelt es sich schließlich um eine politikbegleitende Evaluation, die vor allem als Grundlage für Korrekturen in der Anwendung dient. Die jeweiligen Ziele und die sich daraus ableitenden Fragestellungen müssen möglichst klar und eindeutig formuliert sein.

Ebenso in den Bereich der Vorbereitung der Evaluation fällt die Abgrenzung des Kontextes im Rahmen dessen es nicht nur um die Entwicklung eines Verständnisses für die Motivationen bzw. Zwecke der Evaluation an sich geht, sondern vor allem die herrschenden Ausgangs- sowie Rahmenbedingungen (wie beispielsweise das politische, soziale und wirtschaftliche Umfeld) erfasst werden sollen unter denen die Evaluation stattfindet. Solche Kenntnisse sind von großer Bedeutung für die Gewährleistung einer realistischen und fairen Evaluation und die Anbahnung einer entsprechend aussagekräftigen Interpretation der Ergebnisse.

\section{Auswahl eines Evaluationszeitpunkts}

Im Hinblick auf den Zeitpunkt der Evaluation können im Wesentlichen drei Alternativen unterschieden werden: die summativ-bilanzierende ex-post Evaluation, die formativgestaltende begleitende Evaluation und die präformativ-entwickelnde ex-ante Evaluation. Den Erläuterungen der Projektgruppe Evaluation beim ehemaligen Bundesministerium für Wirtschaft und Arbeit (BMWA) folgend (vgl. BMWA 2004, S. 4f.), werden im Rahmen von ex-post Evaluationen Fragen beantwortet wie beispielsweise ob die gesetzten Ziele mit den gewählten Instrumenten erreicht wurden, die Zielerreichung mit einer effizienten Anwendung der jeweiligen Instrumente umgesetzt wurde und gegebenenfalls welche Konsequenzen sich daraus ergeben.

Im Gegensatz dazu untersucht man im Rahmen von begleitenden Evaluationen ob die eingesetzten Instrumente wie erwartet greifen oder ob sich an der Ausgangslage insoweit etwas geändert hat, dass Ziele und/oder Instrumente angepasst werden müssen. Bei ex-ante Evaluationen ist schließlich zunächst eine Abschätzung der Problemlage notwendig im Rahmen derer zu klären ist, ob und wenn ja wann der Staat wie eingreifen sollte. Im Anschluss daran werden dann - unter Berücksichtigung der Evaluierbarkeit - Ziele und Instrumente festgelegt. 


\subsection{Kriterienset, Vergleichsalternativen und Methoden}

Die Durchführungsebene einer Politikevaluation besteht in der hier vorgeschlagenen Anordnung aus drei zentralen Bausteinen: Der Auswahl eines Kriteriensets, der Festlegung von Vergleichsalternativen und der Auswahl und Anwendung von Indikatoren und Methoden. Diese Bausteine werden im Folgenden in bewährter Weise kurz allgemein erläutert; eine entsprechende Einordnung der bestehenden Arbeiten im Bereich der Wettbewerbspolitik erfolgt dann im anschließenden Kapitel.

\section{Auswahl eines Kriteriensets}

Am Beginn der Durchführungsstufe steht die Notwendigkeit - aufbauend auf der bereits erfolgten Identifizierung des Evaluationsobjekts sowie der Untersuchungsfrage - eine Evaluationsstrategie zu entwickeln. Diese legt im Wesentlichen den Hauptansatz fest, mit der die Untersuchungsfrage beantwortet werden soll (vgl. Bussmann et al., 1997, S. 60). Im Rahmen der Entwicklung einer solchen Strategie ist die Identifikation der Kriterien für die Bewertung des Zielerreichungsgrades von besonderer Bedeutung. In einem ersten Schritt müssen hierzu die zentralen ökonomischen Motivationen, Rechtfertigungen bzw. Ziele für eine entsprechende Politik oder Maßnahme festgehalten werden. Ohne das Vorliegen solcher Gründe ist es nicht möglich, einen positiven Nutzen zu generieren und eine Evaluation wird somit streng genommen überflüssig. In Abhängigkeit des Aggregationsgrades des Evaluationsgegenstands kann diese Zielfindung eine sehr komplexe Aufgabe sein - beispielsweise im Rahmen der Evaluation ganzer Politiken - oder aber zumindest überschaubar, wenn es um die Evaluation von Einzelmaßnahmen geht. Idealerweise werden die entsprechenden Ziele und gegebenenfalls auch zentrale Bewertungskriterien zu Beginn der Politik bzw. Maßnahme festgelegt. Änderungen im Kontext können aber beispielsweise Anpassungen erforderlich werden lassen.

Aufbauend auf der Festlegung des unterstellten Zielsystems muss dann ein Kriterienset erstellt werden. Allgemein gesprochen beschreiben Kriterien Merkmale, mit denen man den Zielerreichungsgrad erfassen kann. Die geeignete Auswahl der Kriterien hängt dabei nahe liegender Weise ab vom Evaluationsgegenstand, dessen unterstelltem Zielsystem sowie der Zielsetzung der Evaluation (d.h. Untersuchung von Wirksamkeit, Wirtschaftlichkeit, Effektivität etc.). Bussmann et al. (1997, S. 72f.) schlagen im Kontext ihrer umfangreichen Erläuterungen zur Politikevaluation vor, ein Wirkungsmodell zu entwerfen, welches die einzelnen Evaluationsgegenstände den einzelnen Evaluationskriterien gegenüberstellt und Hypothesen über deren Zusammenwirken aufstellt. Als jeweilige Paarkombinationen schlagen sie vor: Politikkonzept - Logische Kohärenz des Wirkungsmodells, Policy Design - Kohärenz von Verwaltungsprogrammen, Behördenarrangement für die Politikumsetzung - Eignung der Vollzugsorganisation, Aktionspläne für den Vollzug - Adäquanz der Planung, Outputs - Angemessenheit und Wirtschaftlichkeit der Outputproduktion, Impacts - Effektivität, Outcomes - Wirksamkeit und wirkungsbezogene Wirtschaftlichkeit. Eine solch detaillierte Vorgehensweise erscheint insbesondere bei großen Evaluationsprojekten angebracht zu sein. ${ }^{4}$

\section{Festlegung von Vergleichsalternativen}

Im Anschluss an die Auswahl eines Kriteriensets muss die Festlegung einer Vergleichsalternative - eines so genannten ,counterfactuals' - erfolgen. Denn nur mit Hilfe einer solchen Hilfskonstruktion lässt sich der tatsächliche Zielerreichungsgrad vergleichen mit der hypothetischen Situation, dass entweder gar nichts unternommen worden wäre oder aber eine andere Politik oder Maßnahme implementiert worden wäre. Im Rahmen einer Evaluation kann es dabei durchaus vorkommen, dass die Festlegung von verschiedenen Ver-

\footnotetext{
${ }^{4}$ Ein Beispiel für eine praktische Umsetzung dieses Konzepts wird in Hüschelrath et al. (2009, in dem vorliegenden Sammelband) im Rahmen der Erläuterungen zu Erfahrungen mit der Evaluation des Schweizer Kartellgesetzes gegeben.
} 
gleichsalternativen vonnöten ist. So kann beispielsweise in einem ersten Schritt die Wirkung einer Politik insgesamt untersucht werden und in einem zweiten Schritt der inkrementellen Auswirkung eines Maßnahmenbündels innerhalb dieser Politik nachgegangen werden. Um beide Fälle zu untersuchen müssen unterschiedliche Vergleichsalternativen konstruiert werden.

Im Rahmen der Entwicklung einer Vergleichsalternative kommt der Erlangung eines Verständnisses der Interaktion zwischen verschiedenen Politiken bzw. Maßnahmen eine große Bedeutung zu. Wenn die Interaktionen zwischen verschiedenen Maßnahmen gering sind, so ist es grundsätzlich möglich, die entsprechenden Kosten und Nutzen isoliert zu bestimmen. Im Regelfall wird dies aber nicht der Fall sein und es müssen demnach die wichtigsten Interaktionen zwischen Maßnahmen in die Analyse miteinbezogen werden. Frontier Economics (2006, S. 7ff.) identifiziert in diesem Zusammenhang zwei unterschiedliche Möglichkeiten der Interaktion: Entweder in Form eines so genannten ,Overlapping' - der teilweisen Überlappung zweier Maßnahmen - oder in Form eines sogenannten ,Partnering' - im Rahmen dessen sich beide Maßnahmen gegenseitig ergänzen und zu einem höheren Gesamtnutzen führen als alleine.

Im Anschluss an eine Identifizierung der Vergleichsalternative unter Berücksichtigung der wesentlichen Interaktionen zwischen verschiedenen Maßnahmen ist es dann möglich, den inkrementellen Nutzen der Maßnahme zu bestimmen (vgl. dazu den folgenden Abschnitt).

\section{Auswahl und Anwendung von Indikatoren und Methoden}

Im Anschluss an die Festlegung eines Kriteriensets und der Vergleichsalternativen müssen die Auswahl der Indikatoren und Methoden sowie deren Anwendung erfolgen. Dieser Baustein - gelegentlich auch mit Untersuchungsdesign umschrieben - kann ohne Übertreibung als das Herzstück einer Evaluation bezeichnet werden, da hier letztlich die im Anschluss zu interpretierenden Ergebnisse generiert werden. Bevor eine Methodenauswahl durchgeführt werden kann, sind zunächst im Vorfeld Indikatoren zu bestimmen. Indikatoren verfolgen generell den Zweck, die Umsetzung der Ziele und der Kriterien beobachtbar, messbar und damit überprüfbar zu machen. Sie ermöglichen somit letztlich die gewünschte Ermittlung des Zielerreichungsgrades. Grundsätzlich lassen sich gemäß BMWA (2004) Inputindikatoren, Programmdeskriptoren, Outputindikatoren und Wirkungsindikatoren unterscheiden. Die jeweils geeignete Auswahl wird bestimmt vom jeweiligen Untersuchungsgegenstand, dessen unterstelltem Zielsystem sowie den gesetzten Zielen der Evaluation. Ferner ist darauf zu achten, dass das Indikatorensystem auch wichtige (beabsichtigte oder unbeabsichtigte) Effekte auf weitere Gruppen erfasst, die von der Maßnahme indirekt betroffen sein können. Auch die Berücksichtigung dynamischer und interaktiver Effekte kann eine besondere Herausforderung darstellen im Rahmen der Entwicklung eines Indikatorensystems.

Im Anschluss an die Auswahl der Indikatoren ist über die zur Anwendung kommenden Methoden zu entscheiden. Grundsätzlich stehen qualitative und quantitative Methoden zur Verfügung. ${ }^{5}$ Qualitative Untersuchungen umfassen Beobachtungen (Desk Research), Interviews oder schriftliche Befragungen. Quantitative Methoden erlauben es, Wirkungszusammenhänge auf breiter Basis zu untersuchen. Zu diesen Methoden zählen neben standardisierten Fragebögen, Kosten-Nutzen Analysen, Kosten-Effektivität Analysen oder

\footnotetext{
${ }^{5}$ In Anlehnung an Bussmann (1995, S. 76) muss man genau genommen unterscheiden zwischen Methoden der Datenerhebung (u.a. Dokumentenanalyse, offenes/halboffenes Interview, schriftliche/telefonische/ persönliche Umfrage, statistische Datensammlung, audiovisuelle Aufzeichnung, Gruppengespräch, teilnehmende Beobachtung) und Methoden der Datenauswertung (u.a. Literaturstudien/Dokumentenanalyse, hermeneutische Interpretationsverfahren, Inhaltsanalysen, einfachere statistische Auswertungen, einfache Regressionen, multiple Regressionen, Faktoranalysen, Clusteranalysen, multidimensionale Skalierung, lineare Programmierung, kausale Modellierung mit mathematischen Modellen, ökonometrische Modelle, Simulationen).
} 
Multi-Kriterien Analysen ${ }^{6}$ auch ökonometrische Methoden. Diese bieten insbesondere die Möglichkeit, die Auswirkungen von Politiken oder Maßnahmen unter gleichzeitiger Berücksichtigung anderer Einflussfaktoren auf mikro- oder makroökonomischer Ebene zu analysieren. Solche Methoden sind aber in der Regel komplex und erfordern eine hohe Zahl an Beobachtungen sowie eine Vielzahl typischer, den Analysegegenstand beschreibender Variablen. ${ }^{7}$ In diesem Zusammenhang stellt regelmäßig die Untersuchung der Kausalität - also die Frage, ob der festgestellte Erfolg tatsächlich eine Folge der zu evaluierenden Politik oder Maßnahme ist, oder ob sich der Erfolg auch ohne diese Maßnahme eingestellt hätte - eine besondere Herausforderung dar (vgl. Czarnitzki und Fier, 2005).

\subsection{Ergebnisse, Würdigung und Schlussfolgerungen}

Die Auswertungsstufe einer Politikevaluation besteht aus drei zentralen Bausteinen: Der Ableitung und Interpretation der Ergebnisse, der Würdigung der Aussagekraft sowie der Ableitung von Schlussfolgerungen aus den Evaluationsergebnissen. Diese Bausteine werden im Folgenden in bewährter Weise kurz allgemein erläutert; eine entsprechende Einordnung der bestehenden Arbeiten im Bereich der Wettbewerbspolitik erfolgt dann im anschließenden Kapitel.

\section{Ableitung und Interpretation der Ergebnisse}

Die Ableitung und Interpretation der Ergebnisse schließt sich unmittelbar an die Anwendung der für geeignet befundenen Methoden an. Hierbei kann es in Abhängigkeit der Aufgabenstellung nicht nur notwendig sein, Einzelergebnisse zu interpretieren und in den Kontext zu stellen, sondern es kann auch darum gehen, Aggregationen von Einzelergebnissen zu einem Gesamtergebnis vorzunehmen. Dies ist beispielsweise dann von Nutzen, wenn Informationen über den Netto-Gesamtnutzen von bestimmten Politiken oder Maßnahmen gewünscht werden. In diesem Zusammenhang kann auch die Gewichtung von Einzelkriterien bzw. Ergebnissen eine Bedeutung erlangen. Ferner sollte festgelegt werden, in welcher Form die Ergebnisse (möglicherweise zielgruppenspezifisch) kommuniziert werden sollen. Oftmals ist es auch angebracht, die Präsentation der Ergebnisse im Hinblick auf die Ziele der Evaluation zu interpretieren und gegebenenfalls Verbesserungsvorschläge zu unterbreiten. Zusammenfassend weisen Bussmann et al. (1997, S. 253ff.) darauf hin, dass im Zusammenhang mit der Darstellung der Anwendung quantitativer Verfahrenstechniken und deren Ergebnisse vor allem auf die Klarheit des Berichts, die Offenheit in der Darlegung sowie die Begründung der Folgerungen zu achten ist.

\footnotetext{
${ }^{6}$ Siehe beispielsweise Frontier Economics (2006) für genauere Erläuterungen. Grundsätzlich verfolgen Kosten-Nutzen Analysen das Ziel, Kosten und Nutzenkomponenten monetär zu bewerten und somit vergleichbar zu machen. Dies schließt ausdrücklich Aspekte mit ein, für die ein Markt keine ausreichenden Wertschätzungssignale bereitstellt. Eine Kosten-Effektivität Analyse hingegen vergleicht die Kosten alternativer Wege zu den gleichen Outputs. Sie findet daher oftmals im Rahmen von ex-ante Evaluationen Anwendung. Eine Multi-Kriterien Analyse schließlich beurteilt die Wichtigkeit und den Umfang von Kosten und Nutzen. Sie ist immer dann von besonderem Nutzen, wenn viele Kosten- und Nutzenkomponenten nicht monetär bewertet werden können. Im Rahmen einer solchen Analyse sind die vier Stufen ,Identifizieren-BewertenGewichten-Aufsummieren’ zu durchlaufen.

${ }^{7}$ Die Abschätzung der Vergleichsalternativen ist beispielsweise mit Hilfe ökonometrischer Verfahren möglich. So beschreibt der Vorher-Nachher-Schätzer die Wirkungen einer Politik oder Maßnahme auf beispielsweise Unternehmen nur dann korrekt, wenn sich eine Veränderung der makroökonomischen Rahmenbedingungen nicht auf die Zielvariablen auswirkt oder der Vorher-Zustand der betreffenden Unternehmen nicht schon von der Ankündigung der Politik oder der Maßnahme beeinflusst wird. Mit dem Differenz-derDifferenzen-Schätzer wird zusätzlich eine Vorher-Nachher-Schätzung bei nicht betroffenen Unternehmen durchgeführt und die Differenz der beiden Schätzungen gebildet, um den Einfluss von makroökonomischen Rahmenbedingungen zu korrigieren. Ein möglicher Einfluss der Politik oder der Maßnahme auf den VorherZustand der geförderten Unternehmen kann aber auch hier nicht eliminiert werden (vgl. dazu Czarnitzki et al., 2003).
} 


\section{Würdigung der Aussagekraft}

Der zweite Baustein auf der Auswertungsebene ist die Würdigung der Aussagekraft der erzielten Ergebnisse. Ein zentraler Bestandteil kann die Durchführung von Sensitivitätsüberprüfungen der erzielten Ergebnisse sein. Ferner ist es notwendig, bestehende Unzulänglichkeiten der Analyse klar zu benennen und idealerweise in ihren Wirkungen im Hinblick auf die abgeleiteten Ergebnisse einzuschätzen. Typische Beispiele solcher Unzulänglichkeiten im Rahmen von Evaluationen können ein nicht repräsentativer Ausschnitt bzw. Zeitpunkt der Untersuchung, die Vernachlässigung von wichtigen beeinflussenden (quantifizierbaren oder nicht quantifizierbaren) Faktoren, die unzureichende bzw. inkorrekte Auswahl, Beschreibung oder Anwendung von Methoden, mangelnde Datenverfügbarkeit (und eine somit unvollständige Erfassung der Kosten und Nutzen) sowie eine unzureichende Aufbereitung der Ergebnisse sein. Auch Einschätzungen dynamischer Komponenten sowie bestehender Interaktionen zwischen Politiken bzw. Maßnahmen sind mitunter sinnvoll, sofern diesen Aspekten eine Bedeutung zukommt und eine entsprechende Berücksichtigung im Rahmen der Quantifizierung nicht möglich war.

Bussmann et al. (1997, S. 116) erinnern in diesem Zusammenhang an die Möglichkeit der Durchführung so genannter Metaevaluationen im Rahmen derer die Bewertung von Evaluationsprozessen und der Qualität ihrer Ergebnisse vorgenommen wird. Sie können Aufschluss darüber geben, ob die Ergebnisse einer bestimmten Evaluation wissenschaftlich ausreichend abgesichert sind. Als Grundlage von Metaevaluationen empfehlen Bussmann et al. (1997) die vielfach dokumentierten Evaluationsstandards. ${ }^{8}$

\section{Schlussfolgerungen aus den Evaluationsergebnissen}

Der letzte Schritt einer Evaluation sollte dazu dienen, auf Basis der Ergebnisse Lern- und Implementationsprozesse zu starten und gegebenenfalls Konsequenzen zu ziehen. Die jeweiligen Schlussfolgerungen hängen dabei von den Zielen der Evaluation sowie dem Evaluationstyp ab. Dominierte beispielsweise die Kontrollfunktion im Rahmen einer internen Evaluation, so kann ein wenig erfreuliches Evaluationsergebnis personelle Änderungen nahe legen. War das Ziel eher in einer Prozessverbesserung zu sehen, so muss der Versuch unternommen werden, Vorschläge der Umsetzung zu entwickeln und über deren Implementierung zu beraten. War das Ziel hingehen in einer (zumeist von Externen vorgenommenen) Wirksamkeits- und Wirtschaftlichkeitserhebung zu sehen, so können in Abhängigkeit der Ergebnisse Anpassungen der Politiken, Gesetze oder Maßnahmen erfolgen um diese wirksamer bzw. wirtschaftlicher zu gestalten. Eine weitere - oftmals vernachlässigte - Konsequenz aus Evaluationen können sowohl Änderungen des Kommunikationsverhaltens der Evaluierten sein als auch konkrete (positive oder negative) Einflüsse der Ergebnisse auf die individuellen Arbeitsmotivationen.

\section{Stand der Methodologie zur Evaluation von Wettbewerbspolitik}

Wenngleich die Evaluationsaktivitäten im Bereich der Wettbewerbspolitik in den letzten Jahren spürbar zugenommen haben, so befindet sich die methodologische Diskussion - im Vergleich zu anderen Bereichen wie der Arbeitsmarkt- oder auch Innovationspolitik noch in den Kinderschuhen. Neben einem detaillierten prozessorientierten Beitrag von William Kovacic (2006) beschränkt sich die jüngere Methodologiediskussion im Wesentlichen auf einige Beiträge in einer Sonderausgabe der niederländischen Zeitschrift De Economist aus dem Jahre 2008. ${ }^{9}$

Den Ausführungen des vorangegangenen Kapitels folgend sollte es auch gar nicht das Ziel sein, eine feststehende Methodologie zu entwickeln, solange unterschiedliche Gege-

\footnotetext{
${ }^{8}$ Ausführliche Darstellungen von Evaluationsstandards liefern beispielsweise die Deutsche Gesellschaft für Evaluation (2002) sowie Sanders (2006).

${ }^{9}$ Diese Aufsatzsammlung ist aus einer von der niederländischen Wettbewerbsbehörde NMa im Jahre 2007 veranstalteten Konferenz hervorgegangen.
} 
benheiten und Anforderungen an Evaluationen - zentral beeinflusst durch die jeweils herrschenden institutionellen Bedingungen - vorliegen. Trotz dieser Einschränkung kann es zweifellos von Nutzen sein, Bausteine der Entwicklung einer Evaluationsmethodologie für Wettbewerbspolitik zu identifizieren, die dann - im Stile einer Menüauswahl - für die jeweiligen Evaluationsaufgaben individuell durchdacht und angepasst werden können. Die dazu im vorangegangenen Kapitel aus der einschlägigen Literatur abgeleiteten Bausteine sollen im Folgenden einzeln betrachtet und mit den bestehenden Arbeiten zur Evaluation von Wettbewerbspolitik in Beziehung gesetzt werden. Auf diese Weise können nicht nur zentrale Ergebnisse bestehender Studien systematisch dargestellt werden, sondern es ist gleichzeitig eine Identifizierung von etwaigem Verbesserungspotential möglich.

\subsection{Gegenstand, Ziele und Zeitpunkt}

Die Vorbereitungsstufe einer Politikevaluation besteht in der oben vorgeschlagenen Methodologie aus drei zentralen Bausteinen: Der Identifikation des Evaluationsgegenstands, der Charakterisierung der Funktionen, der Ziele sowie des Kontexts der Evaluation sowie der Auswahl des Zeitpunkts der Evaluation. Basierend auf den generellen Erläuterungen im vorangegangenen Kapitel werden die einzelnen Bausteine im Folgenden in Bezug gesetzt zu bestehenden Arbeiten im Bereich der Wettbewerbspolitik. Bereits an dieser Stelle sei darauf hingewiesen, dass die drei Bausteine im Rahmen der Vorbereitungsstufe nicht zwangsläufig iterativ abgearbeitet werden müssen, sondern dass durchaus eine simultane Betrachtung möglich ist und auch sinnvoll sein kann.

\subsubsection{Identifikation des Evaluationsgegenstands}

Im Hinblick auf eine Evaluation von Wettbewerbspolitik kommt der Identifikation des Untersuchungsgegenstands eine große Bedeutung zu. Dies liegt zu einem großen Teil daran, dass in Abhängigkeit des gewählten Evaluationsgegenstandes teilweise unterschiedliche Anpassungen der weiteren Bausteine der Evaluationsmethodologie erfolgen müssen. Die grundlegenden Überlegungen von Niels and Van Dijk (2008, S. 351ff.) erweiternd lassen sich mindestens sieben mögliche Evaluationsgegenstände im Bereich der Wettbewerbspolitik (im weiteren Sinne) ausmachen: Wettbewerb an sich, die gesamte Wettbewerbspolitik eines Landes oder Staatenverbunds, ein Wettbewerbsgesetz, eine Wettbewerbsbehörde, Teilaktivitäten und Prozesse einer Behörde sowie einzelne Entscheidungen bzw. Entscheidungsbündel einer Behörde.

Die genannten Evaluationsgegenstände unterscheiden sich im Wesentlichen durch die verschiedenen Aggregationsgrade. So kann es im Rahmen eines bottom-up Ansatzes das Ziel sein, eine bestimmte Entscheidung zu analysieren - beispielsweise um deren Korrektheit zu überprüfen und aus etwaigen Fehlern zu lernen oder die volkswirtschaftlichen Auswirkungen zu untersuchen. Es kann aber auch von Interesse sein, dies für ein Entscheidungsbündel oder gar alle Entscheidungen aus einem Bereich der Wettbewerbspolitik (z.B. Fusionskontrolle oder Kartellverfolgung) zu betrachten - beispielsweise um Reformbedarf innerhalb eines solchen Bündels bzw. Bereiches zu ermitteln. ${ }^{10}$ Auf noch höherem Aggregationsniveau ließen sich schließlich auch ganze Wettbewerbsbehörden oder Wettbewerbsgesetze evaluieren. Solche Untersuchungen werden gelegentlich von beaufsichtigenden Behörden gefordert oder finden nach Reformen von Wettbewerbsgesetzen statt - sie werden aber auch von der Organisation for Economic Co-operation and Development (OECD) oder dem privatwirtschaftlichen Global Competition Review (GCR) durchgeführt. Der höchste Aggregationsgrad einer Evaluation ist erreicht, wenn Wettbewerb an sich evaluiert werden soll. Da eine solche Diskussion letztlich im Bereich der Vor- und Nachteile

\footnotetext{
${ }^{10}$ Ein Argument für die Analyse eines Entscheidungsbündels wäre beispielsweise die Beobachtung, dass bestimmte Fälle aufgrund ähnlicher Argumente vor Gericht verloren wurden. Dies legt dann eine genauere Suche nach den Ursachen sowie etwaigen Verbesserungsmöglichkeiten im Rahmen einer Evaluation nahe.
} 
von Wirtschaftsordnungen und Wirtschaftssystemen beheimatet ist, findet sie im Folgenden nur als ,gelegentlich interessanter Extremfall’ Berücksichtigung.

Vergleicht man abschließend die oben angestellten generellen Überlegungen mit der Diskussion in der Wettbewerbpolitik, so fällt ein gewisses Defizit in der Festlegung einer konkreten Forschungs- bzw. Untersuchungsfrage auf, auf die die Evaluation ausgerichtet werden soll. Generell ist anzunehmen, dass mit zunehmendem Aggregationsgrad die Komplexität der Analyse - beispielsweise durch einen Anstieg an Vielfalt der beeinflussenden Faktoren und Ereignisse - zunimmt und demnach die Genauigkeit der Ergebnisse abnimmt. Dadurch wird auch eine Konkretisierung der Forschungs- bzw. Untersuchungsfragen erschwert. In direktem Zusammenhang dazu verändern sich in Abhängigkeit des Aggregationsgrades auch die Schwerpunkte im Bezug auf die Funktionen und Ziele einer Evaluation, die es im folgenden Abschnitt zu untersuchen gilt.

\subsubsection{Funktionen, Ziele und Kontext der Evaluation}

Im Anschluss an eine Festlegung des Evaluationsobjekts muss insbesondere eine Festlegung der Funktionen, der Ziele sowie des Kontextes der Evaluation stattfinden. Im Hinblick auf die Funktionen einer Evaluation von Wettbewerbspolitik lassen sich alle vier oben genannten Funktionen - Erkenntnisfunktion, Kontrollfunktion, Dialogfunktion und Legitimitätsfunktion - zuordnen. So kann eine Evaluation im Wesentlichen die Funktion haben, neue Erkenntnisse zu sammeln, beispielsweise über die Gründe für unterlaufene Fehlentscheidungen oder das Verbesserungspotential behördeninterner Prozesse aufzeigen. Diese Beispiele deuten bereits an, dass es im Rahmen dieser Funktion im Wesentlichen um interne Evaluationen von Gegenständen niedrigen Aggregationsgrades (d.h. Fälle, Prozesse oder Teilbereiche) gehen dürfte, da Analysen auf höherem Aggregationsniveau schnell ein nicht mehr handhabbares Komplexitätsniveau erreichen würden. Buccirossi et al. (2008, S. 454f.) bestätigen diese Sichtweise im Hinblick auf die Evaluation von Fusionsentscheidungen. Sie sehen die Funktion einer solchen Untersuchung im Wesentlichen in der Verbesserung der internen Entscheidungsprozesse in der Fusionskontrolle. Die Analyse vergangener Entscheidungen kann helfen, aus Fehlern zu lernen und die Analysen zu verbessern um auf diese Weise die Anzahl falscher Einscheidungen zu minimieren. ${ }^{11}$

Die Kontrollfunktion einer Evaluation steht der Erkenntnisfunktion sehr nahe, sie ist allerdings naturgemäß mehr mit Erwartungen im Hinblick auf die Ergebnisse verbunden und kann auch direkt organisatorische oder personelle Konsequenzen zu Folge haben. Da eine Zuordnung von Verantwortlichkeiten mit steigendem Aggregationsgrad schwieriger wird, dürfte die Kontrollfunktion ebenso eher auf Fall- bzw. Teilbereichsebene die Hauptrolle spielen. In der wettbewerbspolitischen Literatur spielt diese Funktion eine untergeordnete Rolle - sie mag aber innerhalb von Wettbewerbsbehörden bedeutsam sein.

Die Legitimationsfunktion hat wiederum eine große potentielle Bedeutung für Evaluationen im Bereich der Wettbewerbspolitik. Dies kann alleine schon aus der oftmals bestehenden Verpflichtung der Wettbewerbsbehörden abgeleitet werden, Bericht über die Wirkungen ihrer Tätigkeiten an übergeordnete Behörden zu erstatten. Naturgemäß werden daher eher Gegenstände mit höherem Aggregationsgrad (d.h. Wettbewerbsbehörden oder Wettbewerbsgesetze) im Vordergrund stehen. Niels und van Dijk (2008, S. 350) sehen gerade die Legitimationsfunktion als zentrale Motivation für das festgestellte zunehmende Interesse an Evaluationen von Wettbewerbspolitik in den letzten Jahren. Denn mit Hilfe von Evaluationen kann oftmals beobachtbaren Einwürfen von Zweiflern an der Sinnhaftigkeit solcher Politiken bzw. Maßnahmen entgegengetreten werden.

\footnotetext{
${ }^{11}$ In diesem Zusammenhang sei bereits an dieser Stelle erwähnt, dass Buccirossi et al. (2008, S. 455) zwei zentrale Funktionen in einer ex-post Evaluation von Fusionsentscheidungen ausmachen: „We shall refer to (1) as the 'assessment of the decision with respect to the ultimate economic goal', while we shall refer to (2) as the 'assessment of the analysis that underpins the decision'. Im Rahmen dieses Beitrags wird der Fokus auf der erstgenannten Funktion liegen.
} 
Die Dialogfunktion ist die vielleicht einzige Funktion mit universellem Potential, da sie immer dann eine Rolle spielt, wenn die Ergebnisse der (internen oder externen) Evaluation veröffentlicht werden. Damit ist auch schon angedeutet, dass im Rahmen einer Evaluation durchaus mehrere Funktionen gleichzeitig verfolgt werden können. Da allerdings die Erkenntnisfunktion eher auf niedrigem Aggregationsniveau bedient werden kann, bietet sich eher eine Kombination mit der Kontrollfunktion als mit der Legitimitätsfunktion an. Ein Beispiel für eine Verbindung von Erkenntnis- und Dialogfunktion bietet Sabbatini (2008, S. 494) an. Er verdeutlicht am Beispiel der italienischen Wettbewerbsbehörde, dass für die dortigen Evaluationen von einzelnen Fallenscheidungen zwar das behördeninterne Lernen im Vordergrund stand, durch die Publikation der Ergebnisse aber auch ein externer Beitrag zur Verbesserung der Wettbewerbskultur geleistet werden sollte. Generell erscheint es bedeutsam im Rahmen der Auswahl der Funktion(en) eine klare Strategie zu verfolgen und nicht zu viele Funktionen innerhalb einer Evaluation unterbringen zu wollen.

Die Ziele einer Evaluation von Wettbewerbspolitik sind naturgemäß eng mit den Funktionen verbunden und hängen ebenso vom ausgewählten Evaluationsgegenstand ab. Don et al. (2008, S. 343) nennen vor allem drei Ziele einer Evaluation von Wettbewerbspolitik: das Nachkommen einer bestehenden Rechenschaftspflicht der jeweiligen Wettbewerbsbehörde, eine Qualitätskontrolle der Entscheidungen (mit Hinweisen auf Verbesserungspotential) sowie die Überprüfung der Effektivität des Wettbewerbsrechts.

Neven und Zenger (2008, S. 477) sehen die Zielsetzung einer Evaluation von Wettbewerbsrecht zweigeteilt und etwas fokussierter. Erstens stellt sie ein internes Mittel der Qualitätskontrolle dar, das die Arbeit der Behörde verbessern kann im Bezug auf Prozesse wie auch die Allokation der Ressourcen. Zweitens erlauben solche Studien eine Abschätzung der Kosten und Nutzen von Wettbewerbspolitik und können so für eine externe Qualitätskontrolle genutzt werden wie beispielsweise durch eine beaufsichtigende Behörde oder die interessierte Öffentlichkeit. ${ }^{12}$

Wie bereits im Rahmen der Darstellung der Funktionen verdeutlicht, wird es im Regelfall nicht möglich und sinnvoll sein, alle möglichen Ziele im Rahmen einer Evaluation anstreben zu wollen, sondern es sollte vielmehr in Abstimmung mit den Zielen der passende Evaluationsgegenstand gewählt werden. So wird beispielsweise eine beaufsichtigende Behörde im Regelfall einen Bericht über die Wirksamkeit und Wirtschaftlichkeit aller Aktivitäten erhalten wollen und eine Kommunikation von bestehendem Verbesserungsbedarf mag dabei bestenfalls eine kleine Rolle spielen. Wie bereits oben angedeutet, ist es zwar grundsätzlich möglich, beide Ziele im Rahmen einer Evaluation anzustreben, es gilt aber wiederum der Hinweis, dass mit höherer Aggregationsebene die Komplexität zunehmen und damit die Genauigkeit der Ergebnisse abnehmen dürfte. Vor dem Hintergrund dieses Trade-offs sollte eine Prozessevaluation mit dem Ziel einer Verbesserung der Prozesse eine entsprechend niedrige Aggregationsebene zum Ausgangspunkt nehmen.

Wie implizit an mehreren Stellen bereits angedeutet, muss im Rahmen der Festlegung von Funktionen und Ziele auch eine Entscheidung darüber betroffen werden, ob die Evaluation für interne und/oder externe Zwecke erfolgen soll und ob zur Durchführung interne oder externe Individuen beauftragt werden sollen. Grundsätzlich haben alle vier Paare möglicher Vorgehensweisen ihre Vor- und Nachteile, die es im Hinblick auf die angestrebten Ziele der jeweiligen Evaluation abzuwägen gilt. So haben interne Studien für interne Zwecke durchaus das Potential, Erkenntnisfortschritte für interne Prozessverbesserungen zu liefern, da die Evaluierenden mit den Prozessen in der Regel gut vertraut sind. Nachteile könnten aber in einer gewissen Betriebsblindheit sowie der fehlenden Bereitschaft liegen,

\footnotetext{
${ }^{12}$ Ein OECD Roundtable zum Thema Evaluation von Wettbewerbspolitik stellte insbesondere zwei Motivationen von Evaluationen in den Vordergrund: “... (a) to examine the effectiveness of existing management practices, organisational structure, operational procedures, and (b) to study the impact of specific interventions, such as the prosecution of cases, the filing of reports, and the pursuit of advocacy initiatives“ (OECD, 2005, S. 7).
} 
Kollegen mit einem wenig positiven Evaluationsergebnis zu konfrontieren. Die Alternative einer externen Studie für interne Zwecke löst das letztgenannte Problem, schafft aber dafür unter Umständen ein neues Problem durch die unzureichende Sach- und Detailkenntnis der externen Gutachter. Darüber hinaus kann eine externe Studie auch deshalb problematisch sein, weil Externe aus rechtlichen Gründen nicht mit allen (z.B. fallbezogenen) Daten ausgestattet werden können, die sie für eine vollständige Analyse bräuchten. Eine interne Studie für externe Zwecke hat den Vorteil, dass ausgehend von einer guten Datenbasis eine kompetente Analyse möglich wäre, ein Nachteil ist es aber, dass Wettbewerbsbehörden wohl mit der Kommunikation negativer Evaluationsergebnisse an die Öffentlichkeit in der Regel vorsichtig sein werden. Insbesondere das Eingestehen falscher Entscheidungen ist in diesem Zusammenhang als problematisch anzusehen, da die seinerzeit betroffenen Unternehmen eine Klage gegen die Behörde in Erwägung ziehen könnten (vgl. dazu auch Bergman, 2008). Vor diesem Hintergrund ist es sehr wahrscheinlich, dass die Wettbewerbsbehörde in der ex-post Analyse zu dem gleichen Ergebnis kommt, wie seinerzeit in der Fallentscheidung, unabhängig davon, ob die Entscheidung nun richtig war oder nicht. Dies mag auch ein Grund dafür sein, dass ex-post Evaluationen von Behörden meist davon ausgehen, dass alle Entscheidungen richtig waren. Die Vor- und Nachteile der letzten möglichen Kombination - eine externe Studie für externe Zwecke - lassen sich dann im Wesentlichen aus den bisherigen Erläuterungen ableiten. Ein bislang nicht genanntes Argument sieht die größere Methodenkompetenz in der Regel bei Externen, insofern wäre eine solche Studie auch aus diesem Blickwinkel heraus möglicherweise glaubwürdiger als eine InHouse Untersuchung. Dem steht möglicherweise entgegen, dass auch Externe vor zu deutlicher Kritik zurückschrecken könnten um eine mögliche Zusammenarbeit mit der Wettbewerbsbehörde in der Zukunft - beispielsweise als Berater - nicht zu gefährden (vgl. dazu Kovacic, 2006, S. 520). Insgesamt gesehen erscheint es aufgrund der festgestellten Vorund Nachteile ratsam für eine Wettbewerbsbehörde, sowohl interne als auch externe Evaluationen durchzuführen um auf diese Weise Komplementaritäten zwischen beiden Studienarten ausnutzen zu können.

Im Rahmen der abschließenden Beschreibung des Kontextes sollen unter anderem die herrschenden Ausgangs- sowie Rahmenbedingungen - wie beispielsweise das politische, soziale und wirtschaftliche Umfeld - erfasst werden unter denen die Evaluation stattfindet. Dieser Bereich ist als sehr projektspezifisch anzusehen und eine Einschätzung kann nur direkt für den jeweiligen Fall vorgenommen werden. Als ein praktisches Beispiel kann in diesem Zusammenhang die EU Kommission herangezogen werden, die sich vor einigen Jahren einer Reihe von verlorenen Gerichtsentscheiden in wichtigen Wettbewerbsfällen ausgesetzt sah und als Reaktion auf die Kritik an der unzureichenden bzw. unvollständigen ökonomischen Argumentation sowohl organisatorische als auch gesetzgeberische Änderungen umsetzte. Für eine etwaige Evaluation wäre der Kontext einer Reihe von verlorenen Gerichtsentscheiden sicherlich bedeutsam im Hinblick auf die Planung und Durchführung der Evaluation.

Als ein weiteres Beispiel kann hier angeführt werden, dass sich die Schwerpunkte bzw. die Bedeutung bestimmter Evaluationsgegenstände und -ziele zwischen einzelnen Ländern deutlich unterscheiden können. So mag es für Länder mit einer geringen Erfahrung im Bereich des Wettbewerbsrechts bedeutsamer sein, die entsprechenden Prozesse zu evaluieren und infolgedessen zu verbessern, während es in wettbewerbsrechtlich erfahreneren Ländern eher darauf ankommen kann, den konkreten volkswirtschaftlichen Nutzen der Unterhaltung von Wettbewerbspolitik zu dokumentieren. ${ }^{13}$ Wie bereits in der Einleitung ange-

\footnotetext{
${ }^{13}$ Grundsätzlich ist es aber umgekehrt denkbar, dass gerade in Ländern mit einer kurzen Historie von Wettbewerbspolitik notwendig ist, etwaigen Zweiflern mit Hilfe einer Evaluation auf hoher Aggregationsebene zu verdeutlichen, welche Nutzen eine effizient implementierte Wettbewerbspolitik der Gesellschaft bringt. Eine ähnliche Situation könnte in Ländern bestehen, in denen in den letzten Jahren eine umfassende Reform des Wettbewerbsrechts stattgefunden hat (vgl. dazu Hüschelrath et al., 2009).
} 
sprochen ist ein solcher Trend derzeit in Europa spürbar. Etwaigen Schlussfolgerungen, dass Wettbewerbspolitik für den Steuerzahler ,ein teures Vergnügen' sei, soll dann durch solche Dokumentationen der Vorteile entgegengetreten werden. Verstärkend kann wirken, wenn ein schlechtes wirtschaftliches Umfeld die entsprechenden Regierungen zum Sparen zwingt und daher möglicherweise mehr Politiken und Maßnahmen auf den Prüfstand kommen als dies in wirtschaftlich besseren Zeiten der Fall wäre. Ähnliches gilt für Regierungs- bzw. Politikwechsel mit denen möglicherweise eine Änderung der wettbewerbspolitischen Ausrichtung einhergeht.

\subsubsection{Auswahl eines Evaluationszeitpunkts}

Im Hinblick auf eine Evaluation von Wettbewerbspolitik ist die Wahl des Zeitpunkts von unterschiedlicher Bedeutung und wiederum abhängig von dem zu Beginn definierten Evaluationsgegenstand. Ist eine Evaluation von Wettbewerb, Wettbewerbspolitik oder Wettbewerbsrecht insgesamt geplant, so wird streng genommen eine ex-post Untersuchung gar nicht möglich sein, da nicht deren Abschaffung ernsthaft diskutiert wird. In einem solchen Umfeld spielen somit im Regelfall begleitende Evaluationen die Hauptrolle. Im Falle der Evaluation von Teilgebieten der Wettbewerbspolitik ist die Sachlage nicht mehr ganz so eindeutig, da es zumindest für kleinere Länder durchaus gute Gründe geben kann, beispielsweise über die Abschaffung der Fusionskontrolle nachzudenken und eine Korrektur etwaig entstehender marktmächtiger Positionen mit Hilfe der Missbrauchskontrolle vorzunehmen. Sollte ein solcher Schritt umgesetzt werden, so wäre anschließend eine ex-post Evaluation der Wirkungen und der Wirtschaftlichkeit möglich. Die Hauptrolle einer expost Evaluation spielt allerdings auf der Ebene der Entscheidungen von Wettbewerbsbehörden. Diese sind in der Regel abgeschlossen und folglich einer ex-post Evaluation zugänglich. Neven und Zenger (2008, S. 477) definieren folglich ex-post Evaluation auch als Vergleich des Ergebnisses eines Wettbewerbseingriffs mir der Vergleichsalternative dass dieser Eingriff nicht stattgefunden hätte.

Die ex-ante Evaluation spielt in den einzelnen Untersuchungsgegenständen möglicherweise die geringste Rolle. Sie könnte zum Einsatz kommen, wenn Änderungen in Erwägung gezogen werden und man im Vorfeld (im Rahmen der Entscheidungsvorbereitung) abschätzen möchte, ob und wie eine entsprechende Wirksamkeit und Wirtschaftlichkeit erreicht werden könnte. Ein Beispiel wäre die Frage, ob eine Wettbewerbsbehörde ein neues Instrument (z.B. eine Kronzeugenregelung für Kartellanten) einführen sollte oder nicht. Auf der Fallebene freilich spielt ex-ante Evaluation insofern eine sehr große Rolle, als diese letztlich die Hauptaufgabe einer Wettbewerbsbehörde im Bereich der Fusionskontrolle darstellt. $^{14}$

In diesem Zusammenhang vertritt Sabbatini (2008, S. 494f.) interessanterweise eine etwas abweichende Interpretation von ex-ante Evaluation, in dem er alle wettbewerbsbehördlichen Analysen darunter fasst, in denen ein Fall entschieden ist aber die Marktwirkungen noch unklar sind und diese deshalb prognostiziert werden sollen. Eine solche Analyse nehmen beispielsweise die Wettbewerbsbehörden in den USA regelmäßig vor, wenn sie mit Hilfe einfacher Formeln die Effekte ihrer Entscheidungen abzuschätzen versuchen. ${ }^{15}$ Unter einer ex-post Untersuchung versteht Sabbatini (2008, S. 496) die Überprüfung der Qualität der Entscheidungen sowie gegebenenfalls der Effektivität der von der Wettbewerbsbehörde unternommenen Aktivitäten.

\footnotetext{
${ }^{14}$ Allerdings handelt es sich hierbei um keine Evaluation im Sinne dieser methodologischen Untersuchung, sondern schlicht um originäre Entscheidungen einer Behörde die anschließend einer Evaluation unterliegen (beispielsweise durch Gerichte oder Forschungsinstitute).

${ }^{15}$ Eine genauere Beschreibung des Ansatzes erfolgt in Abschnitt 3.2.3.
} 


\subsection{Kriterienset, Vergleichsalternativen und Methoden}

Die Durchführungsebene einer Politikevaluation besteht in der hier vorgeschlagenen Anordnung aus drei zentralen Bausteinen: Der Auswahl eines Kriteriensets, der Festlegung von Vergleichsalternativen und der Auswahl und Anwendung von Indikatoren und Methoden. Diese Bausteine werden im Folgenden in bewährter Weise in Bezug gesetzt zu bestehenden Arbeiten im Bereich der Wettbewerbspolitik.

\subsubsection{Auswahl eines Kriteriensets}

Eine unverzichtbare Grundlage für jedwede Form der Evaluation von Wettbewerbspolitik ist größtmögliche Klarheit über die entsprechenden Ziele von Wettbewerbspolitik. Vorab sei in diesem Zusammenhang darauf hingewiesen, dass man klar unterscheiden muss zwischen den Zielen der Evaluation an sich (vgl. dazu Abschnitt 3.1.2 oben) sowie den Zielen des Evaluationsgegenstands, die in diesem Abschnitt betrachtet werden sollen.

Ein geeigneter Start in eine solche Betrachtung stellen Definitionsversuche der Ziele von Wettbewerbspolitik dar. So sieht beispielsweise Werden (2008, S. 433) die Ziele insbesondere darin, Aktivitäten abzuschrecken, die lediglich der Beschränkung oder Ausschaltung des Wettbewerbs dienen und solche Aktivitäten mit Auflagen zu versehen, die ohne diese Auflagen negative Wirkungen entfalten würden. Obwohl diese Definition zweifellos den Kern der Zielsetzung wettbewerbspolitischer Aktivitäten trifft, so fällt bei genauerer Betrachtung auf, dass sie nur schwer operationalisierbar ist im Rahmen einer Evaluation. Denn letztlich müsste untersucht werden, inwiefern ein solcher optimaler Grad an Abschreckung realisiert ist. ${ }^{16}$ In einem solchen Kontext müsste man aktuelle Eingriffe also beispielsweise aufgedeckte Kartelle und untersagte Fusionen - streng genommen als negativen Output der Wettbewerbsbehörde werten, da die Abschreckung in diesen Fällen offensichtlich nicht die gewünschte Wirkung entfalten konnte. ${ }^{17}$

Aus dieser Gemengelage lässt sich der Wunsch nach einer operationalisierbareren Zielsetzung wettbewerbspolitischer Aktivitäten ableiten. Aus wirtschaftspolitischer Sicht wird Wettbewerb überwiegend nicht als Selbstzweck begriffen, sondern als Mittel zur Stimulierung ökonomisch effizienter Verhaltensweisen, die sich dann in der Folge wiederum positiv auf Wirtschaftswachstum, Produktivität und somit die gesamtwirtschaftliche Wohlfahrt auswirken (vgl. van Sinderen und Kemp, 2008, S. 376ff.). Von einem Großteil der wissenschaftlich tätigen Ökonomen wird daher das Hauptziel von Wettbewerbspolitik in der Wohlfahrtsmaximierung gesehen, oder genauer formuliert in der Maximierung der positiven Wirkungen der wettbewerbspolitischen Instrumente auf die Wohlfahrt. Die sich anschließende Frage, was genau unter Wohlfahrt zu verstehen sein soll, ist dabei allerdings umstritten. So geht die Mehrzahl der Ökonomen grundsätzlich davon aus, dass Wettbewerbspolitik auf eine Maximierung der Gesamtwohlfahrt ausgerichtet sein sollte, also komparativ-statische Änderungen auf Basis der inkrementellen Effekte auf die Summe von Produzentenrente und Konsumentenrente beurteilt werden sollten.

Ein zentrales Problem eines solchen Standards ist allerdings, dass er im Rahmen von Anwendungen nicht ausschließt, dass sich marktmächtige Unternehmen auf Kosten der Konsumenten einen größeren Teil der zu vergebenden Gesamtrente eines Marktes sichern und auf diese Weise zu einer wachsenden Asymmetrie in der Vermögensverteilung beitragen. Geht man von einer geplanten Fusion als Beispiel aus, so ist es im Rahmen der Anwendung eines Gesamtwohlfahrtsstandards durchaus möglich, dass die Fusion genehmigt wird obwohl sich die Konsumenten in der Folge Preiserhöhungen aufgrund der gestiege-

\footnotetext{
${ }^{16}$ Über mögliche Methoden einer Messung des Abschreckungseffekts informiert Abschnitt 3.2.3.

${ }^{17}$ An dieser Stelle sei der Hinweis gestattet, dass dies nicht zwangsläufig der Fall sein muss, da es aus Kosten-Nutzen-Erwägungen heraus einen optimalen Grad an Wettbewerbsvergehen gibt. Dieser wird typischerweise nicht an einem Punkt erreicht, an dem es keinerlei Wettbewerbsvergehen mehr gibt, sondern liegt an der Stelle an der die marginalen Kosten und die marginalen Nutzen eines weiteren Ausbaus der Verfolgung von Wettbewerbsvergehen gleich sind.
} 
nen Marktmacht der fusionierenden Parteien ausgesetzt sehen. Einzige Bedingung für eine solche Genehmigung wäre der Nachweis von realisierbaren Effizienzvorteilen auf Seiten der fusionswilligen Parteien (mindestens) in Höhe der Einbußen an Konsumentenrente in der Folge der Fusion. Wenngleich eine solche Situation - in der Literatur bekannt als ,Williamson Trade-off' - aus gesamtwirtschaftlicher Sicht effizient im Sinne von wohlfahrtserhöhend wäre, so wäre sie für eine Wettbewerbsbehörde nur schwer zu kommunizieren ${ }^{18}$ (vgl. van Sinderen und Kemp, 2008, S. 376ff.). Insofern wird in der aktuellen Diskussion von den Wettbewerbsbehörden selbst wie auch von vielen Wissenschaftlern davon ausgegangen, dass das Ziel von Wettbewerbspolitik eher in der Maximierung der Konsumentenwohlfahrt liegt.

Für die Verwendung eines solchen Konsumentenwohlfahrtsstandards gibt es durchaus weitere gute Argumente. So differenzieren Besanko und Spulber (1993) beispielsweise zwischen einer prozeduralen und einer konzeptionellen Wahl des Wohlfahrtsstandards. Sie zeigen, dass es aufgrund der bestehenden Informationsasymmetrien zwischen den Unternehmen und der Wettbewerbsbehörde die volkswirtschaftlich beste Wahl sein kann, einen Konsumentenwohlfahrtsstandard zu verfolgen da auf diese Weise ein gewisses Gegengewicht für die unternehmensseitig bestehenden Informationsvorteile geschaffen wird und somit im Gesamtergebnis das Ziel der Gesamtwohlfahrtsmaximierung am Besten erreicht wird. Eine vergleichbare Argumentation liefern Neven und Röller (2006), die Lobbyingvorteile auf Seiten der Unternehmen im Vergleich zu den Konsumenten als Rechtfertigung für die Verfolgung eines Konsumentenwohlfahrtsstandards sehen. Ein sehr praktisches Argument für die Verfolgung eines Konsumentenwohlfahrtsstandards entwickelt Werden (1996). Er ist der Auffassung, dass Wettbewerbsbehörden deshalb einen Konsumentenwohlfahrtsstandard verfolgen sollten, weil er leicht zu definieren und leicht zu kommunizieren ist.

Hüschelrath (2009b) präsentiert ein weiteres praktisches Argument, das die Nutzung eines Konsumentenwohlfahrtsstandards durch die Wettbewerbsbehörden erklärt: Im Falle eines Konsumentenwohlfahrtsstandards ist der ausgewiesene Nutzen der wettbewerbsbehördlichen Aktivitäten deutlich höher als im Falle der Konzentration auf die Gesamtwohlfahrt. In manchen Industrien mit sehr preisunelastischer Nachfrage - wie beispielsweise der Energieversorgung - wären die durch Wettbewerbspolitik verhinderten NettoWohlfahrtsverluste sehr gering, die Umverteilungseffekte aber entsprechend groß. Würde man nur die Netto-Nutzen im Rahmen einer Evaluation als Nutzen begreifen - so wie dies der Gesamtwohlfahrtsstandard vorsieht - und den Kosten gegenüberstellen, so wäre es sehr viel schwieriger, eine Kostendeckung zu erreichen als im Rahmen eines Kosumentenwohlfahrtsstandards. ${ }^{19}$

Selbst eine Einigung auf einen Konsumentenwohlfahrtsstandard als Wohlfahrtsmaß löst nicht alle Probleme, die im Rahmen der Festlegung eines Kriteriensets für eine Evaluation auftreten. So muss beispielsweise genauer definiert werden, was unter Konsumentenwohlfahrt verstanden werden soll und welche Determinanten in eine Quantifizierung einfließen sollen. Die meisten Studien konzentrieren sich dabei auf die Auswirkungen von Veränderungen im Marktpreis auf die Konsumentenrente. Dies ist insofern unbefriedigend, als ne-

\footnotetext{
${ }^{18}$ Renckens (2007) verdeutlicht mit Hilfe eines einfachen graphischen Modells die Unterschiede, die sich bei der Anwendung der verschiedenen Standards ergeben. So müssen bei einem reinen Preisstandard alle Fusionen untersagt werden, die zu einer Preissteigerung führen unabhängig davon, wie groß die Ersparnisse der Unternehmen sein mögen. Ein Konsumentenwohlfahrtsstandard ist diesem sehr vergleichbar. Der zentrale Unterschied zum Preisstandard liegt darin, dass Effizienzvorteile mit in die Betrachtung einbezogen werden und demnach eine Fusion erlaubt wird, wenn sie zwar die Marktmacht erhöht aber die Preise aufgrund der realisierten Effizienzen nicht steigen.

${ }^{19}$ An dieser Stelle wird bereits offensichtlich, welche Anreizwirkungen Evaluationen haben können. Würde die Arbeit einer Wettbewerbsbehörde beispielsweise nach einem Gesamtwohlfahrtsstandard evaluiert, so hätte die Behörde einen Anreiz, ihre Untersuchungen in preisunelastischen Märkten zurückzufahren oder gar einzustellen und dafür in preiselastischeren Märkten zu intensivieren.
} 
ben dem Preis weitere Faktoren wie Qualität, besserer Service oder die Einführung neuer Produkte einen Wert für die Konsumenten haben, der letztlich im Rahmen der entsprechenden Analysen berücksichtigt werden muss. Das zentrale Ergebnis einer diesbezüglichen FIPRA-Studie (vgl. Evans, 2008) zeigt, dass die Konsumentenwohlfahrt in der kurzen Frist zwar stark vom Preis beeinflusst wird, aus mittel- und langfristiger Perspektive der Konsumentenwohlfahrt aber am ehesten gedient ist mit einer Ausrichtung des unternehmerischen Wirkens auf Effizienz und Innovation. ${ }^{20}$

Vor diesem Hintergrund schlagen van Sinderen und Kemp (2008, S. 266ff.) vor, das Zielsystem von Wettbewerbspolitik breiter anzulegen und deutlicher die mittel- und langfristigen Ziele des Wettbewerbs im Bezug auf höheres Wachstum, höhere Produktivität und höhere Beschäftigung einzubeziehen. Denn alle diese Faktoren führen letztlich zu neuen Produkten und verbesserten Produktionsprozesse und erhöhen somit die Konsumentenwohlfahrt.

Besagte dynamische Aspekte des Wettbewerbs sind es auch, die Niels und van Dijk (2008, S. 350) allerdings vom Einstimmen in das Hohelied des reinen Konsumentenwohlfahrtsstandards in der Wettbewerbspolitik abhalten. ${ }^{21}$ Sie argumentieren, dass die Wettbewerbsbehörden zwar in den letzten Jahren erfolgreiche die ,consumer welfare card' ausgespielt haben, dass diese aber durchaus auch Nachteile hat. So kann ein solcher Standard falsche Erwartungen wecken und Aktionen von Wettbewerbsbehörden herausfordern, immer dann wenn Konsumenten scheinbar oder aktuell von Unternehmen ausgenommen werden. Solcher Aktionismus könnte zumindest aus dynamischer Sicht deutlich negative Folgen haben. ${ }^{22}$

Im Einklang mit ihrer kritischen Einschätzung im Hinblick auf die Wünschbarkeit eines reinen Konsumentenwohlfahrtsstandards im Rahmen der Wettbewerbspolitik weisen Niels und van Dijk (2008, S. 353) am Beispiel einer Kosten-Nutzen Analyse darauf hin, dass es von großer Bedeutung ist, die Kosten und Nutzen aller wichtigen Beteiligten in die Analyse einfließen zu lassen, d.h. Konsumenten, Produzenten, Regierung, Steuerzahler etc. Dabei kann allerdings eine Gewichtung der jeweiligen Kosten und Nutzen vorgenommen werden. Die folgende Tabelle 1 zeigt eine Übersicht der anzunehmenden Kosten und Nutzen, die zunächst für jedweden Evaluationsgegenstand in Erwägung gezogen werden sollten. Diese können als Kriterien im Sinne dieses Abschnitts interpretiert werden.

\footnotetext{
${ }^{20}$ Die Notwendigkeit einer weiten Interpretation des Begriffs der Konsumentenwohlfahrt verdeutlicht auch das OFT (2008b, S. 7f.) in einer jüngeren Studie: “Consumer benefit does not arise solely from actions that remedy price raising anticompetitive activity. Direct consumer benefits may also arise from a market study that facilitates switching, enforcement of consumer law or education activities that lead consumers to make improved choices. Financial benefits to consumers include: decrease in price, monetized improvements in quality range or service, monetized time savings, and the benefits that consumers gain from making better informed choices about what goods to purchase."

${ }^{21}$ Grundsätzlich sind neben den reinen Konsumenten- bzw. Gesamtwohlfahrtsstandards auch Mischungen beider über die Gewichtung der Bedeutung von Konsumenten- und Produzentenrenten möglich (vgl. dazu u.a. Hüschelrath, 2009a, S. 62f.).

${ }^{22}$ In diesem Zusammenhang sprechen sich Niels und van Dijk (2008) konsequent auch gegen simple Aufrechnungen von beispielsweise Strafen für Kartelle und den Budgets der jeweiligen Wettbewerbsbehörden aus. Denn im Falle eines Gesamtwohlfahrtsstandards handelt es sich dabei um wohlfahrtsneutrale Transfers die folglich in einer Kosten-Nutzen-Analyse keine Rolle spielen.
} 


\begin{tabular}{l|l}
\hline Costs & Benefits \\
\hline Direct (administrative) costs of the authority & \\
\hline Direct costs of firms & \\
Regulatory compliance costs & \\
Costs of specific competition proceedings & \\
\hline Economic costs to the market in question & Economic benefits to the market in question \\
(negative market impacts) & (positive market impacts) \\
Allocative inefficiency & Allocative efficiency \\
Productive inefficiency & Productive efficiency \\
Distortion of incentives (reduced dynamic & Enhanced dynamic \\
competition/innovation) & competition/innovation \\
Reduced product/service quality & Increased product/service quality \\
Restriction on market functioning & Enhanced market functioning \\
\hline Indirect regulatory costs & Indirect regulatory benefits \\
Regulatory uncertainty & Regulatory certainty \\
Likelihood of regulatory capture & Deterrent effects \\
- Social costs (if relevant) & Improved quality of regulation \\
Distributive costs & Social benefits (if relevant) \\
Reduced security/quality of supply & Distributive benefits \\
Negative effect on vulnerable customers & Enhanced security/quality of supply \\
Other negative externalities on society & Positive effect on vulnerable customers \\
\hline Quelle. Oxera (2004) & Other positive externalities on society \\
\hline
\end{tabular}

Quelle: Oxera (2004)

Bislang nicht detailliert angesprochen aber von besonderer Bedeutung in der obigen Tabelle ist die Vielfalt an möglichen Kosten der Wettbewerbspolitik die im Rahmen einer Evaluation in die Wohlfahrtsbetrachtung einfließen müssen. Neben den direkten Kosten der Wettbewerbsbehörde fallen auch auf Unternehmensseite Kosten - beispielsweise in Form von Complianceaktivitäten - an. Daneben können auch Kosten entstehen durch unscharfe Gesetze und demzufolge suboptimale strategische Entscheidungen der Unternehmen aus Angst vor einem wettbewerbsrechtlichen Regelverstoß. Ein weiterer möglicher Kostenblock ist in Einflüssen unberechtigter wettbewerbspolitischer Interventionen zu sehen, also Fehlern von Wettbewerbsbehörden (vgl. dazu auch Niels und van Dijk, 2008, S. 356).

Generell ist im Hinblick auf die oben identifizierten Evaluationsgegenstände anzumerken, dass die Auswahl der Kriterien sich an ihnen orientieren sollte. So ist es vermutlich wenig sinnvoll, die Auswirkungen einer Entscheidung auf das Wachstum der Volkswirtschaft untersuchen zu wollen, da diese im Regelfall nicht bedeutend genug sein werden, um einen isolierbaren Effekt bei makroökonomischen Variablen zu hinterlassen. In solchen Fällen bieten sich dann eher detaillierte Analysen der Effekte auf die statische und dynamische Konsumentenrente bzw. die Gesamtwohlfahrt an. Umgekehrt wird es im Rahmen einer Abschätzung der Kosten und Nutzen von Wettbewerbspolitik kein realistisches und erstrebenswertes Ziel sein, die Effekte aller Entscheidungen der Wettbewerbsbehörde zu analysieren, sondern es ist eine bestimmte Auswahl von Musterfällen ratsam.

Aus methodologischer Sicht ist abschließend der Hinweis wichtig, dass eine aktuelle Evaluation von den Zielen und Einschränkungen ausgehen muss, die zum Zeitpunkt der Entscheidung bestanden haben. So wäre es nicht korrekt, heute einen Konsumentenwohlfahrtsstandard anzuwenden, wenn dieser zum Zeitpunkt der Entscheidung so gar nicht bestanden hatte oder sein Einsatz von anderen Faktoren eingeschränkt wurde. Eine solche Analyse wäre immer als verzerrt und unausgewogen anzusehen (vgl. dazu auch Neven und Zenger, 2008, S. 481). Angesichts der dynamischen Weiterentwicklungen im Bereich der Wettbewerbspolitik in den vergangenen Jahren kann dies einen potentiell großen Einfluss auf Evaluationen und ihre Ergebnisse haben. Um nachträgliche vergangenheitsorientierte Zieldiskussionen zu vermeiden ist es daher ratsam, die Ziele von Politiken bzw. Maßnahmen - sowie möglicherweise auch gleich Schlüsselindikatoren zur Erfolgsmessung (vgl. 
dazu Abschnitt 3.2.3 unten) - im Vorfeld festzulegen. Von besonderer Bedeutung ist eine solche Festlegung freilich, wenn die Kontrollfunktion einer Evaluation eine bedeutende Rolle spielt und möglicherweise Personalentscheidungen mit dem Ergebnis der Evaluation verknüpft sind.

\subsubsection{Festlegung von Vergleichsalternativen}

Im Anschluss an eine Auswahl des Kriteriensets muss eine Festlegung der Vergleichsalternativen erfolgen um dann in der Folge den tatsächlichen Zielerreichungsgrad vergleichen zu können mit der hypothetischen Situation, dass entweder gar nichts unternommen worden wäre oder aber eine andere Politik oder Maßnahme implementiert worden wäre. Da Vergleichsalternativen definitionsgemäß nicht beobachtet werden können, müssen sie konstruiert werden. So muss beispielsweise im Falle einer untersagten Fusion untersucht werden, was passiert wäre, wenn die Fusion genehmigt worden wäre. Da im Rahmen einer solchen hypothetischen Abschätzung das aktuelle Marktgeschehen keine hinreichende Orientierungshilfe leisten wird, stellt die Konstruktion der Vergleichsalternative(n) eine der größten Herausforderungen im Rahmen einer Evaluation dar.

Vor dem Hintergrund der verschiedenen Evaluationsobjekte (vgl. dazu Abschnitt 3.1.1 oben) ist es unmittelbar einsichtig, dass die Vergleichsalternativen entsprechend individuell zugeschnitten werden müssen. Den detaillierten Überlegungen von Niels und van Dijk (2008, S. 350) folgend, ließe sich auf höchster Ebene grundsätzlich an eine Evaluation von Wettbewerb denken. Wie oben bereits angedeutet, müssten im Rahmen einer solchen Untersuchung dann andere Wirtschaftsordnungen bzw. -systeme die Vergleichsalternativen bilden. Wenngleich diese theoretische Option im Folgenden nicht weiterentwickelt werden soll, so lassen sich mit ihrer Hilfe zwei zentrale Punkte verdeutlichen. So ist einerseits eine Evaluation von Wettbewerbspolitik nicht gleichzusetzen mit einer Evaluation von Wettbewerb. Die Unterschiede in beiden Betrachtungsebenen ergeben sich schon daraus, dass auch ohne Wettbewerbspolitik in vielen Märkten Wettbewerb weiter bestünde. Andererseits tragen auch viele andere Politiken wie Handelspolitik oder Deregulierungspolitik zum Nutzen von Wettbewerb insgesamt bei. In gewisser Weise ist es ja ,nur' die Aufgabe von Wettbewerbspolitik, Wettbewerb zu schützen - was implizit seine Existenz voraussetzt. Dies verdeutlicht direkt auch das im allgemeinen Teil oben angesprochene Problem der Überlappung verschiedener Politiken und einer entsprechend schwierigen Isolierung der Effekte einzelner Politiken.

Die nächste Stufe in der Systematik von Niels und van Dijk (2008, S. 352) ist die Unterscheidung zwischen einer Evaluation von Wettbewerbspolitik bzw. Wettbewerbsrecht und einer Evaluation einer Wettbewerbsbehörde. So wäre die relevante Vergleichsalternative für die Evaluation einer legislativen Änderung die Fortführung der alten Regelung bzw. alternative Änderungen. Für den Fall einer Evaluation der Aktivitäten einer Wettbewerbsbehörde wäre die relevante Vergleichsalternative hingegen nicht zwangsläufig ein Zustand ohne Wettbewerbspolitik, sondern durchaus auch eine Verfolgung der Wettbewerbsregeln auf Basis rein privatrechtlicher Klagen. Daraus folgt dann unmittelbar, dass alle Nutzen, die auch in einem System privatrechtlicher Durchsetzung realisiert würden, sich folglich nicht dem Wirken der Wettbewerbsbehörde als Nutzen zuschreiben ließen. In einem solchen Szenario wäre demnach unter anderem die Frage zu beantworten, welche Formen von Wettbewerbsbeschränkungen eher an Bedeutung gewinnen und welche eher an Bedeutung verlieren würden in einem System privatrechtlicher Durchsetzung des Wettbewerbsrechts. Niels und van Dijk (2008, S. 357) zeigen, dass dies unter anderem davon abhängt, ob Wettbewerber bzw. Konsumenten die Wettbewerbsbeschränkung bemerken sowie wie sie ihre Chancen und Möglichkeiten der Durchsetzung einschätzen. Auf Basis dieser beiden Kategorien identifizieren die Autoren einige Bereiche, die mit großer Wahrscheinlichkeit bei einer rein privatrechtlichen Durchsetzung von Wettbewerbsrecht keine Rolle spielen würden. Darunter fallen letztlich alle Wettbewerbsbeschränkungen in Endverbraucher- 
märkten, also Preisabsprachen sowie Fusionen als auch Marktmachtmissbrauch. Die dort von einer Wettbewerbsbehörde generierten Nutzen sind uneingeschränkt der Existenz der Behörde zu verdanken, da Konsumenteninteressen tendenziell diffus und daher als wenig einflussreich angesehen werden müssen. Auch in Märkten für Geschäftskunden (so genannten Business-to-Business-Märkten) würden Absprachen wahrscheinlich nur entdeckt, wenn die jeweiligen Abnehmer groß und gut informiert sind. Auch hier kann die Existenz einer Wettbewerbsbehörde Vorteile bringen.

Auf unterster Aggregationsebene ist schließlich auf die Konstruktion von Vergleichsalternativen auf der Ebene der Fallentscheidungen bzw. Bündel von Fallentscheidungen einzugehen. Ein Großteil der verfügbaren Literatur beschäftigt sich mit der Konstruktion von Vergleichsalternativen auf dieser Ebene, wobei im Regelfall Fusionen im Zentrum des Interesses stehen. Im Folgenden sollen allerdings neben Fusionen auch Kartelle im Hinblick auf die Konstruktion von Vergleichsalternativen charakterisiert werden.

Im Hinblick auf die Konstruktion von Vergleichsalternativen für die ex-post Evaluation von Fusionen muss zunächst zwischen drei möglichen Entscheidungen der Wettbewerbsbehörde unterschieden werden: Einer Freigabe ohne Auflagen, einer Freigabe mit Auflagen sowie der Untersagung des Fusionsvorhabens. Diesen Alternativen zuzuordnen ist jeweils das Verhalten der Fusionsparteien, das jeweils entweder im Vorschlagen von Auflagen oder dem Verzicht eines solchen Vorschlags besteht. Die jeweiligen Ausprägungen dieser beiden Kategorien erlauben dann die Zuordnung der jeweiligen Vergleichsalternative(n), die in der nachfolgenden Tabelle 2 dargestellt sind.

\section{Tabelle 2: Vergleichsalternativen zur ex-post Evaluation von freigegebenen und un- tersagten Fusionen}

\begin{tabular}{|c|c|c|}
\hline $\begin{array}{l}\text { Entscheidung der Wettbe- } \\
\text { werbsbehörde }\end{array}$ & Verhalten der Fusionsparteien & Vergleichsalternative \\
\hline \multicolumn{3}{|l|}{ Freigabe ohne Auflagen } \\
\hline & Keine Auflagen vorgeschlagen & Untersagung \\
\hline & Auflagen vorgeschlagen & $\begin{array}{l}\text { Untersagung } \\
\text { Freigabe unter Auflagen }\end{array}$ \\
\hline \multirow[t]{2}{*}{ Freigabe mit Auflagen } & & \\
\hline & $\begin{array}{l}\text { Keine Auflagen vorgeschlagen } \\
\text { Auflagen vorgeschlagen }\end{array}$ & $\begin{array}{l}\text { Untersagung } \\
\text { Freigabe ohne Auflagen }\end{array}$ \\
\hline \multirow[t]{2}{*}{ Untersagung } & & \\
\hline & $\begin{array}{l}\text { Keine Auflagen vorgeschlagen } \\
\text { Auflagen vorgeschlagen }\end{array}$ & $\begin{array}{l}\text { Freigabe ohne Auflagen } \\
\text { Freigabe ohne Auflagen } \\
\text { Freigabe mit Auflagen }\end{array}$ \\
\hline
\end{tabular}

Quelle: Buccirossi et al. (2008, S. 457)

Wie Tabelle 2 verdeutlicht, ist die einzig mögliche Vergleichsalternative für den Fall einer Freigabe ohne Auflagen und ohne Vorschlag von Auflagen seitens der Fusionsparteien die Untersagung. Eine Freigabe unter Auflagen ist in diesem Szenario keine gangbare Alternative, da angenommen wird, dass es der Wettbewerbsbehörde nicht möglich ist, selbst Auflagen vorzuschlagen, sondern dass dieser Schritt nur von den fusionierten Parteien selbst in Form eines Einbringens eines Vorschlags wie die Wettbewerbsprobleme geheilt werden können - getätigt werden kann. Eine solche Vorgehensweise ist beispielsweise in der Europäischen Union gesetzlich festgelegt. Besagte Regel erklärt auch unmittelbar, weshalb für den Fall einer Freigabe mit Auflagen ohne Vorschlag von Auflagen seitens der Fusionsparteien die Ableitung einer Vergleichsalternative nicht möglich ist (vgl. dazu detaillier Buccirossi et al., 2008). Wie Tabelle 2 weiter verdeutlicht, weisen alle Kombinationen in denen von Seiten der Fusionsparteien Auflagen vorgeschlagen wurden, eine besondere Komplexität auf. Dies liegt einfach daran, dass in diesen Fällen nicht nur jeweils zwei Vergleichsalternativen zur Auswahl stehen, sondern letztlich eine sehr große Anzahl von 
alternativen Auflagen verglichen werden müssten. Wie Buccirossi et al. (2008) aber ausführen, kristallisieren sich in der Praxis oftmals einige wenige gangbare Wege ab, die dann im Rahmen einer ex-post Betrachtung analysiert werden können.

Im Falle einer ex-post Evaluation von Kartellentscheidungen stellt sich die Ausgangslage ungleich einfacher dar als im Falle von Fusionen. Dies hat zentral damit zu tun, dass es im Falle von Kartellen im Regelfall unstrittig ist, dass ein solches vorgelegen hat und darüber hinaus auch von Seiten des Gesetzes im Rahmen eines per-se Verbotes angenommen wird, dass solche Wettbewerbsbeschränkung ausschließlich negative Wohlfahrtswirkungen haben. Mit anderen Worten ausgedrückt kann es im Rahmen einer Evaluation nicht darum gehen, ähnliche Szenarien wie im Falle von Fusionen zu kreieren, da weder das Vorliegen der Wettbewerbsbeschränkung noch die Entscheidung der Wettbewerbsbehörde im Regelfall strittig sind. ${ }^{23}$ Die Festlegung einer Vergleichsalternative ist aber dennoch notwendig, da nur auf diese Weise die Frage untersucht werden kann, wie der entsprechende Markt ohne die Kartellabsprache ausgesehen hätte und welche Änderungen in den Wohlfahrtswirkungen dies zur Folge hat. Dabei ist nicht unmittelbar einsichtig, welches die richtige Betrachtungsrichtung ist. So kann man einerseits mit einer solchen Analyse das rückwärtsgewandte Ziel verfolgen, den Schaden den das Kartell vom Bestehen bis zu seiner Aufdeckung verursacht hat, zu messen. Andererseits wäre es möglich, der vorwärtsgewandten Frage nachzugehen, welche Schäden in der Zukunft aufgetreten wären, hätte die Wettbewerbsbehörde nicht interveniert. Für eine Evaluation von Wettbewerbspolitik erscheint die zweite, vorwärtsgewandte Betrachtungsrichtung die geeignetere zu sein, während die erstgenannte eher im Rahmen der Bestimmung der Schadenshöhe und Sanktionsbemessung zum Einsatz kommen dürfte.

Vergleicht man abschließend die generellen Überlegungen zur Festlegung von Vergleichsalternativen mit der Diskussion in der Wettbewerbpolitik, so stößt ein gewisses Defizit in der Betrachtung von Überschneidungen verschiedener Politiken ins Auge. So beeinflussen sich verschiedene Maßnahmen innerhalb der Handels-, Wettbewerbs- und Deregulierungspolitik oft gegenseitig und müssten demnach im Rahmen einer Evaluation auch eingeschätzt werden. In diesen Bereich würde es auch fallen, die Existenz und den inkrementellen Nutzen verschiedener Wettbewerbsgesetze innerhalb eines Landes sowie das etwaige Vorliegen verschiedener Wettbewerbsbehörden innerhalb eines Landes zu betrachten. Zumindest zu letzterem Punkt hat es in der Vergangenheit einige Meinungsaustausche in den USA gegeben, die sich um die Frage gedreht haben, welche Vor- und Nachteile die Aufrechterhaltung zweier Wettbewerbsbehörden beinhaltet. Ähnliche Situationen liegen in Großbritannien und mit Abstrichen auch in Deutschland vor und würden eine genauere Untersuchung nahe legen.

\subsubsection{Auswahl und Anwendung von Indikatoren und Methoden}

Im Anschluss an die Festlegung eines Kriteriensets und der Vergleichsalternative(n) müssen die Auswahl der Indikatoren und Methoden sowie deren Anwendung erfolgen. Indikatoren verfolgen generell den Zweck, die Umsetzung der Ziele und der Kriterien beobachtbar, messbar und damit überprüfbar zu machen. Es müssen Entscheidungen darüber getroffen werden, welche der im Kriterienset als grundsätzlich relevant befundenen Kriterien für die vorliegende Evaluation auszuwählen sind und wie diese gemessen werden sollen. Im Anschluss an die Auswahl der Indikatoren ist dann über die zur Anwendung kommenden Methoden zu entscheiden. Grundsätzlich stehen hier qualitative und quantitative Methoden zur Verfügung. Aus Gründen der gebotenen Knappheit sollen im Folgenden nicht alle in

\footnotetext{
${ }^{23}$ Letztlich muss diese Sichtweise allerdings als vereinfachend deklariert werden, da es beispielsweise durchaus sein kann, dass eine Wettbewerbsbehörde zwar keine Fehler bei der Aufdeckung des Kartells begeht, aber dafür beispielsweise eine inkorrekte Strafzumessung vornimmt. Von solchen Fällen soll im Folgenden aber abstrahiert werden. Darüber hinaus kann eine Wettbewerbsbehörde auch im Bereich der Kartellverfolgung streng genommen ,Fehler’ begehen, in dem sie bestehende Kartelle nicht aufdeckt.
} 
Abschnitt 3.1.1 identifizierten Evaluationsobjekte genauer untersucht werden, sondern es soll sich im Wesentlichen auf zwei Objekte konzentriert werden: Einerseits eine Evaluation von Wettbewerbspolitik an sich, sowie andererseits eine Evaluation von Fällen bzw. Fallbündeln (differenziert nach Fusionen und Kartellen). Diese Trennung erweist sich auch deshalb als zweckmäßig, weil sich die Mehrzahl der bestehenden Studien diesen beiden Aggregationsebenen zuordnen lässt. Entgegen der bisherigen Vorgehensweise soll im Folgenden zunächst die Evaluation von Fällen bzw. Fallbündeln behandelt werden und erst im Anschluss die Evaluation der gesamten Wettbewerbspolitik. Dies hat zentral damit zu tun, dass viele Studien auf einer höheren Aggregationsebene teilweise auf die (quantitativen und qualitativen) Methoden der niedrigeren Aggregationsebene zurückgreifen.

\subsubsection{Fälle bzw. Fallbündel als Evaluationsgegenstand}

Die Evaluation von Fällen oder Fallbündeln kann verschiedenen Zielsetzungen unterliegen. Die detaillierten Überlegungen in Abschnitt 3.1.2 zusammenfassend sind in diesem Zusammenhang insbesondere die interne Qualitätskontrolle mit dem Ziel einer Prozessverbesserung sowie die externe Qualitätskontrolle mit dem Ziel der Rechenschaftsablegung zu nennen. Im Folgenden soll aus Vereinfachungsgründen angenommen werden, dass es das Hauptziel einer ex-post Untersuchung von Fällen bzw. Fallbündeln ist, die internen Prozesse zu evaluieren und gegebenenfalls zu verbessern. Dabei soll vor dem Hintergrund des in Abschnitt 3.2.1 abgeleiteten Kriteriensets davon ausgegangen werden, dass die Wettbewerbsbehörde einen Konsumentenwohlfahrtsstandard verfolgt. Da die Höhe der Konsumentenrente in der kurzen Frist vor allem vom Marktpreis, dem Transaktionsvolumen sowie der Qualität und Vielfalt der jeweiligen Produkte abhängt, steht die Messung dieser Indikatoren im Mittelpunkt der folgenden Abschnitte. Grundsätzlich müssen allerdings auch weitere - insbesondere langfristig wirksame - Einflussfaktoren auf die Konsumentenwohlfahrt in die Analyse einbezogen werden. Eine solche Erfassung ist aber oft nur indirekt möglich, indem beispielsweise Änderungen in der Marktstruktur, des Marktverhaltens oder des Marktergebnisses beobachtet, mit der jeweiligen Fallentscheidung in Verbindung gebracht und dann Rückschlüsse auf solch mittel- und langfristige Einflussfaktoren getroffen werden. Den genaueren Ausführungen von Sabbatini (2008, S. 492) folgend ist ferner darauf hinzuweisen, dass sich die ex-post Betrachtungen zwischen Fusionen und Kartellen insbesondere dahingehend unterscheiden, als erstere eine Änderung der Marktstruktur betreffen, während die Aufdeckung von Kartellen kurzfristig eher Änderungen im Marktverhalten erwarten lässt. Zumindest mittel- und langfristig löst sich diese Trennung allerdings auf, da auch in Folge der Aufdeckung eines Kartells durchaus Markteintritte denkbar sind und somit Änderungen in der Marktstruktur ausgelöst werden können. Weitere zentrale Unterschiede in der Behandlung von Fusionen und Kartellen ergeben sich aus den bereits im vorangegangenen Abschnitt 3.2.2 angesprochenen Unterschieden im Hinblick auf mögliche Fehlentscheidungen der Wettbewerbsbehörde.

\subsection{Methoden zur Evaluation von Fusionsentscheidungen}

Ausgehend vom Ziel der Fusionskontrolle - der Maximierung der Konsumentenwohlfahrt - lässt sich zunächst allgemein feststellen, dass der Zielerreichungsgrad typischerweise mit fallendem Marktpreis, steigendem Marktvolumen und steigender Qualität bzw. Vielfalt wächst. Die zentrale Aufgabe einer Evaluation ist nun auf Basis dieser Indikatoren zu untersuchen, wie sich diese seit der Entscheidung verändert haben und wie sie sich vermutlich unter der bzw. den Vergleichsalternativen entwickelt hätten. Ausgehend von der in Abschnitt 3.2.2 wiedergegebenen Tabelle 2 schlagen Buccirossi et al. (2008) folgerichtig für jeden Fall eine methodische Untersuchungsstrategie vor. Exemplarisch sollen hier nur der erste Fall (Freigabe ohne Auflagen - keine Auflagen vorgeschlagen - Untersagung) 
sowie der vorletzte Fall (Untersagung - keine Auflagen vorgeschlagen - Freigabe ohne Auflagen) kurz skizziert werden. ${ }^{24}$

Im Fall des Vorliegens einer Freigabe ohne Auflagen ohne das Angebot von Fusionsauflagen ist die einzig mögliche Vergleichsalternative die Untersagung, da es der Wettbewerbsbehörde annahmegemäß nicht möglich ist, selbst Auflagenvorschläge in den Verhandlungsprozess einzubringen. Daraus folgt, dass Erkenntnisse über die Konsumentenrentenunterschiede zwischen der Situation bei erfolgter Fusion und der (hypothetischen) Situation nach untersagter Fusion erlangt werden müssen. Eine solche Identifikation ist in zwei Schritten möglich. In einem ersten Schritt betrachtet man die Änderungen der zentralen Indikatoren wie Preise, Mengen, Qualität bzw. Vielfalt im Zeitablauf. Stellt man im Rahmen einer solchen Analyse beispielsweise fest, dass die Preise nach der Fusion gefallen sind, so legt das insofern eine effektive Entscheidung der Wettbewerbsbehörde nahe, als die Konsumentenwohlfahrt ceteris paribus in der Folge der Fusion angestiegen ist. Da die beobachteten Änderungen der Indikatoren aber nicht zwangsläufig auf die Fusion zurückzuführen sein müssen, sondern auch von externen Faktoren verursacht sein können, muss in einem zweiten Schritt mit Hilfe ökonometrischer Methoden untersucht werden, inwieweit sich eine Kausalität zwischen der Entwicklung der zentralen Konsumentenrentenindikatoren und der Fusion belegen lässt. In diesem Zusammenhang stellen Buccirossi et al. (2008, S. 460) eine ganze Liste an möglichen Hypothesen und dazugehöriger empirischer Untersuchungsstrategien zusammen. Konkret könnte beispielsweise einerseits die Frage zu untersuchen sein, ob eine beobachtete Verschiebung der Nachfragefunktion ihre Ursache in einer Verbesserung der Qualität bzw. Vielfalt durch die Fusion hat oder nicht. Alternativ wäre eine Untersuchung der Frage möglich, ob die bei der fusionierten Unternehmung festgestellte Produktionskostensenkung auch bei anderen Unternehmen im Markt in ähnlicher Größenordnung beobachtet werden kann.

Der zweite hier kurz zu skizzierende Fall - eine Untersagungsentscheidung ohne das Angebot von Fusionsauflagen - ist deutlich komplizierter als die Untersuchung einer Freigabe. Dies hat zentral damit zu tun, dass die Marktentwicklung im Anschluss an die Untersagung nicht bei der Ableitung einer Hypothese hilft, die anschließend gestestet werden könnte. Während im vorangegangenen Fall der Untersuchungsschwerpunkt darin lag herauszufinden, ob die betreffende Fusion der Treiber der beobachtbaren Änderungen der Indikatoren war, so ist diese Vorgehensweise im Falle einer Untersagungsentscheidung aufgrund der Unmöglichkeit der Beobachtung der Marktentwicklung - nicht anwendbar. Es ist daher in einem solchen Rahmen nur möglich, einen kausalen Zusammenhang zwischen einer (hypothetischen) Fusion und ihren (hypothetischen) Effekten zu definieren und zu testen.

Basierend auf diesem auszugsweise geschilderten Untersuchungsdesign erläutern Buccirossi et al. (2008, S. 464ff.) zentrale empirische Methoden, die bei einer ex-post Analyse von Fusionen Anwendung finden können. Im Wesentlichen sind dies Simulationen, die Schätzung struktureller Modelle, Natürliche Experimente und Matching-Methoden, Ereignisstudien sowie Befragungen. Da diese Methoden nicht nur im Rahmen der Fusionsevaluation, sondern beispielsweise auch im Bereich der Kartelle Anwendung finden können, sollen sie im Folgenden jeweils kurz charakterisiert werden. Dabei fließen auch die Sichtweisen anderer Kommentatoren in die auf Buccirossi et al. (2008) basierenden Einschätzungen ein.

Explizit sei an dieser Stelle bereits der Hinweis gestattet, dass alle der im Folgenden zu erläuternden Methoden ihre Vor- und Nachteile haben und daher nicht in eine Rangfolge

\footnotetext{
${ }^{24}$ Die Untersuchungen in den beiden ausgewählten Fällen sind insofern als ,relativ’ einfach einzuschätzen, als sie nicht eine oder mehrere Alternativauflagen in die Analyse mit einbeziehen müssen. Wenngleich sich an der grundlegenden Analyse und den angewandten Methoden auch in solchen Fällen nicht viel ändert, so wird die gesamte Analyse doch ungleich komplexer als für die beiden hier betrachteten Fälle ohne vorgeschlagene Auflagen (vgl. dazu detailliert Buccirossi et al., 2008).
} 
gebracht werden können. Ferner ist auch nicht jede Methode gleich gut für die Erreichung einer bestimmten Zielsetzung einsetzbar, sondern die Auswahl hängt neben der Fragestellung auch von der Art der Entscheidung, den Marktcharakteristika sowie den verfügbaren Daten ab. Idealerweise kommen mehrere Methoden zur Beantwortung einer bestimmten Fragestellung zum Einsatz um auf diese Weise die Robustheit der Ergebnisse zu überprüfen.

\section{Simulationen}

Allgemein gesprochen nutzen Simulationsprogramme einfache Oligopolmodelle um mit ihnen hypothetische Änderungen in der Marktstruktur (z.B. Fusionen oder im Rahmen von Auflagen zu veräußernder Unternehmen(steile)) abzubilden und im Hinblick auf ihre Auswirkungen auf die zu erwartenden Marktergebnisse einschätzen zu können (siehe z.B. Werden und Froeb 1994, 2002 sowie Epstein und Rubinfeld, 2001). Im Kontext der expost Evaluation von Fusionen können solche Programme beispielsweise genutzt werden, um für den Fall der Untersagung einer Fusion den hypothetischen Preisanstieg im Falle einer Genehmigung zu berechnen. Abgeglichen mit den tatsächlichen Marktpreisen und dem Marktvolumen lässt sich dann ein erster Wohlfahrtseffekt der Entscheidung ermitteln.

Zur Verdeutlichung der Grundidee solcher Simulationen sei im folgenden beispielhaft angenommen, dass im Rahmen einer ex-post Evaluation einer Untersagungsentscheidung der Effekt einer Freigabe der Fusion als Vergleichsalternative bestimmt werden soll. Geht man davon aus, dass sich der entsprechend zugrunde liegende Markt durch ein homogenes Cournot-Modell gut abbilden lässt, so lässt sich der hypothetische prozentuale Preisanstieg mit einer einfachen Formel abschätzen. Dazu wird von der folgenden allgemeinen Beziehung zwischen dem Lerner-Index und dem Herfindahl-Hirschman Index ausgegangen:

$$
\mathrm{L}=\sum_{\mathrm{i}} \frac{\mathrm{m}_{\mathrm{i}}^{2}}{\varepsilon_{\mathrm{D}}^{\mathrm{m}}}=\frac{\mathrm{HHI}}{\varepsilon_{\mathrm{D}}^{\mathrm{m}}} .
$$

Gleichung (1) besagt, dass der Lerner-Index für einen Markt bzw. eine Industrie gleich ist dem entsprechenden Wert des HHI dividiert durch die Marktnachfrageelastizität. Inhaltlich bedeutet dies, dass Marktmacht mit steigender Konzentration eines Marktes ansteigt und mit steigender Marktnachfrageelastizität fällt. Durch Umformung von Gleichung (1) lässt sich dann eine einfache Formel für die Abschätzung des Preisanstiegs in der Folge einer Fusion ableiten:

$$
\frac{\Delta \mathrm{p}}{\mathrm{p}}=\frac{\Delta \mathrm{HHI}}{\varepsilon_{\mathrm{D}}^{\mathrm{m}}-\mathrm{HHI}_{\text {postmerger }}} .
$$

Wie Gleichung (2) verdeutlicht, ist der prozentuale Preisanstieg einer Fusion gegeben durch die fusionsinduzierte absolute Änderung des HHI dividiert durch die um den HHI Wert nach der Fusion reduzierte Marktnachfrageelastizität. Nimmt man beispielsweise für die Marktnachfrageelastizität einen Wert von 1 an, so lassen sich die verschiedenen Gebiete von prozentualen Preiserhöhungen - in Abhängigkeit des HHI Wertes nach der Fusion sowie des fusionsinduzierten Änderung des HHI - graphisch darstellen. Dies wird in der nachfolgenden Abbildung 2 verdeutlicht. 


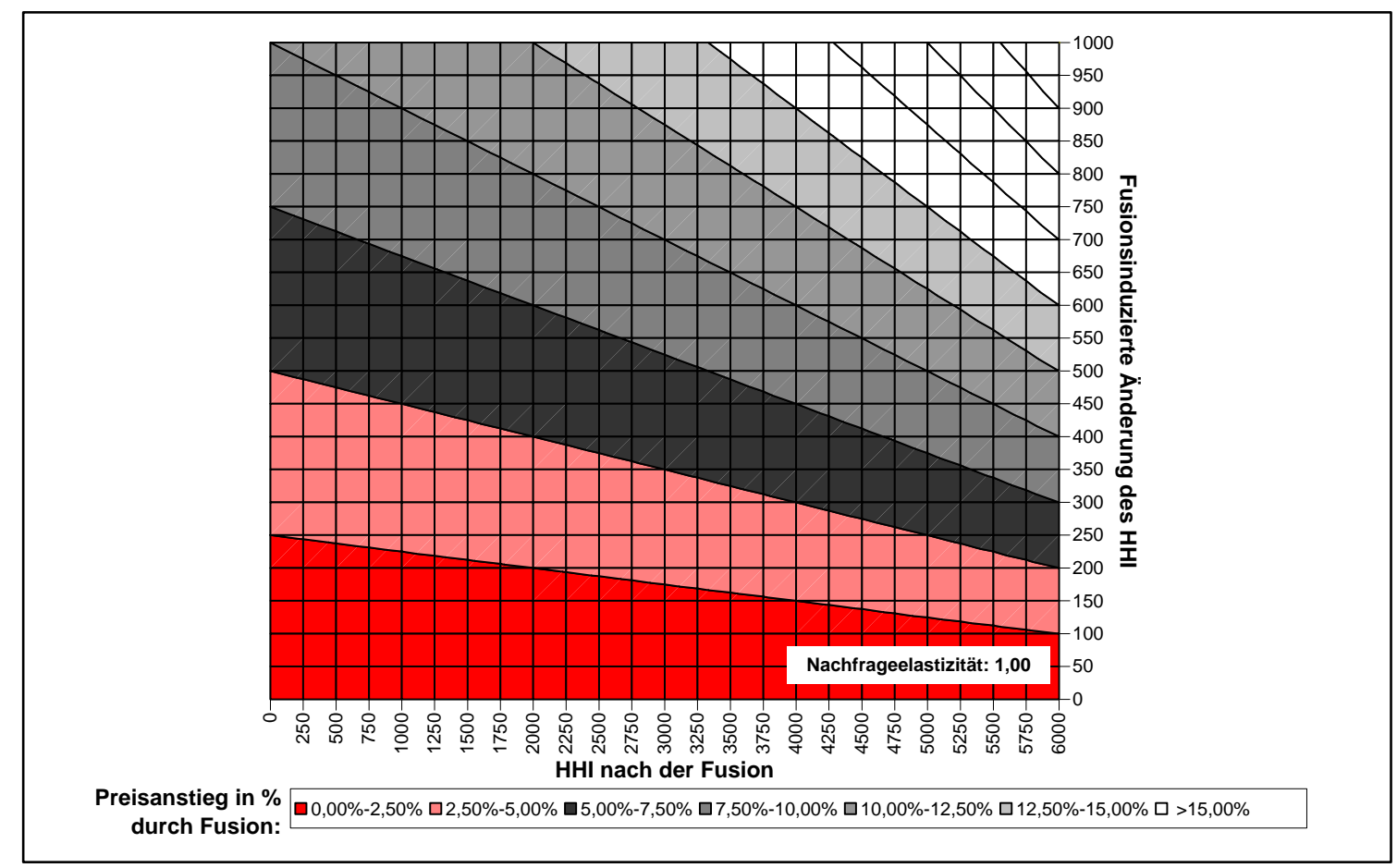

Abbildung 2: Simulation des fusionsinduzierten prozentualen Preisanstiegs

Abbildung 2 zeigt den fusionsinduzierten prozentualen Preisanstieg für verschiedene Kombinationen von HHI's nach der Fusion sowie fusionsinduzierten Änderungen des HHI's. So wird beispielsweise für einen HHI nach der Fusion von 3000 und einer fusionsinduzierten Änderung des HHI von 450 ein Preisanstieg zwischen 5,00\% und 7,50\% vorhergesagt (der exakte Wert kann zu 6,43\% durch Einsetzen in die Formel ermittelt werden). Hierbei ist allerdings darauf hinzuweisen, dass die Berechnung (sowie die gesamte Abbildung 2) auf der Annahme einer Preiselastizität von 1,0 basiert. Nimmt man für das gleiche Zahlenbeispiel eine Preiselastizität der Marktnachfrage von 1,5 an, so ergibt sich lediglich noch einen Preisanstieg von 3,75\% in Folge der Fusion. Dies verdeutlicht die Sensitivität der Ergebnisse im Hinblick auf die unterstellte Elastizität.

Die Einfachheit der obigen Analyse sollte aber nicht darüber hinweg täuschen, dass sie auf einer Vielzahl sehr vereinfachender Annahmen basiert. Neben den bereits genannten Annahmen von Mengenwettbewerb und homogenen Gütern zählen zu diesen Annahmen beispielsweise, dass alle Unternehmen konstante Grenzkosten haben, die Fusion die Kostenstruktur der beteiligten Unternehmen unverändert lässt oder auch dass die Fusion das Verhalten der Unternehmen im Markt unverändert lässt. Einleitend erwähnte professionelle Fusionssimulationsprogramme sind allerdings in der Lage, viele dieser sehr vereinfachenden Annahmen aufzulösen und in komplexere Modelle zu integrieren (vgl. dazu genauer Hüschelrath, 2010). Dabei soll allerdings nicht verschwiegen werden, dass auch diese Modelle teilweise deutlicher Kritik durch Wissenschaftler ausgesetzt sind und diverse Überprüfungen von Prognosen der Simulationsmodelle mit der dann eingetretenen Wirklichkeit nicht immer befriedigende Ergebnisse erzielt haben (vgl. dazu u.a. Peters, 2006, Weinberg, 2006, Slade, 2006 und Crooke et al., 1999). Angesichts der Komplexität und Unvorhersehbarkeit zukünftiger Marktentwicklungen ist dies aber ein Problem, mit welchem letztlich alle Methoden in irgendeiner Weise zu kämpfen haben.

\section{Strukturelle Modelle}

Strukturelle Modelle folgen dem Paradigma der 'New Empirical Industrial Organization' und basieren auf der Idee, die Parameter eines Sets von Strukturgleichungen - die den betreffenden Markt unter Beobachtung gut beschreiben - zu schätzen. Diese Gleichungen 
werden abgeleitet mit Hilfe spieltheoretischer Oligopolmodelle. Die geschätzten Parameter werden dann herangezogen um mögliche Zukunftsszenarien zu simulieren. Die Parameter können erst im Rahmen einer ex-ante Evaluation geschätzt werden und dann genutzt werden um begründete Vorhersagen zu den Auswirkungen nach Durchführung der Fusion zu machen.

Die Schätzungen auf der Basis eines strukturellen Modells lassen sich grob in vier Schritte unterteilen: In einem ersten Schritt erfolgt die Schätzung der Nachfrage sowie die Berechnung der Elastizitäten, gefolgt von einer Ermittlung der Grenzkosten im zweiten Schritt. Im dritten Schritt erfolgt dann die Simulation des neuen Preisgleichgewichts auf der Basis der geschätzten Nachfrage sowie der Grenzkosten unter den für geeignet befundenen Annahmen zum Preisverhalten nach der Fusion. In einem vierten Schritt erfolgt dann auf Basis der geschätzten Preisänderungen die Schätzung der Wohlfahrteffekte.

Buccirossi et al. (2008) diskutieren die Vor- und Nachteile struktureller Modelle im Rahmen der ex-post Evaluation von Fusionsentscheidungen. Sie sehen zentrale Vorteile in der klaren spieltheoretischen Fundierung, den Möglichkeiten der Modellierung verschiedener Spezifikationen von Nachfrageseite und wettbewerblicher Interaktion sowie den allgemein guten Möglichkeiten zur Durchführung von Sensitivitätsanalysen. Ein Problem der Schätzung struktureller Modelle kann generell im Trade-off bestehen zwischen Anwendungsfreundlichkeit einerseits und der gewünschten Präzision der Schätzergebnisse andererseits. Weitere Nachteile können gesehen werden in den hohen Anforderungen an die benötigten Daten sowie der Vielzahl an Annahmen, die sowohl im Theorie- als auch im Empirieteil in die Analyse einfließen. Insofern muss bei der Anwendung des Modells auf die Daten darauf geachtet werden, dass die theoretischen Annahmen auf den zu analysierenden Markt möglichst gut zutreffen. Da die Ergebnisse oft stark von den zentralen Annahmen abhängen, sind Robustheitsüberprüfungen obligatorisch.

\section{Natürliche Experimente und Matching-Methoden}

Natürliche Experimente und Matching-Methoden basieren beide auf einem Vergleich von zwei Gruppen: einer so genannten ,control group' und einer so genannten ,experimental group’ (vgl. dazu Buccirossi et al, 2008, S. 466f.). Die beide Methoden verbindende Grundidee dabei ist, dass der Unterschied in den realisierten Ergebnissen zwischen den beiden Gruppen als Abschätzung für die Effekte des Markteingriffs angesehen werden kann. Natürliche Experimente erfordern einen natürlichen unbeeinträchtigten Markt bzw. Unternehmen, die allerdings dem Fusionsmarkt sehr ähnlich sind (d.h. zum Beispiel denselben Angebots- und Nachfragebedingungen unterliegen). Die Matching-Methode versucht, die betroffenen Unternehmen (also Fusionsparteien und Wettbewerber) mit Unbetroffenen zu vergleichen anhand einiger beobachtbarer und als bedeutsam eingeschätzter Kriterien. Ein beide Methoden verbindendes Problem ist das einer möglicherweise vorliegenden Endogenität, das heißt der Möglichkeit, dass andere nicht beobachtete Parameter die Entscheidung zur Fusion beeinflusst haben und auch die Ergebnisse der Fusionsparteien beeinflussen. Ein genereller Vorteil beider Methoden ist, dass sie häufig und flexibel einsetzbar sind. Die Aussagekraft der erzielten Ergebnisse hängt aber zentral von der geeigneten Wahl der Vergleichsmärkte bzw. -unternehmen ab.

\section{Ereignisstudien}

Ereignisstudien messen auf Basis von Kapitalmarktdaten die Auswirkungen spezifischer Ereignisse auf den Unternehmenswert. Die zentrale Annahme hinter Ereignisstudien sind effiziente Kapitalmärkte, d.h. alle für die Marktteilnehmer relevanten und öffentlich verfügbaren Informationen schlagen sich unverzüglich und vollständig in den Preisen nieder. Der Kurs einer Aktie spiegelt somit die Bewertung aller relevanten Informationen über die Fundamentaldaten einer Unternehmung wider. Positive oder negative Informationen, die 
den Markt erreichen, führen zu einer Kursreaktion. Dessen isolierte Betrachtung ermöglicht die Messung der Eigenkapitaländerung.

Im Kontext von Fusionsentscheidungen nutzen Ereignisstudien die Reaktion der Aktienkursentwicklung der Wettbewerber auf die Fusionsankündigung für eine Evaluation der Entscheidungen der Wettbewerbsbehörden. Ist eine Fusion wettbewerbsfreundlich in dem Sinne, dass sie zu Effizienzen führt die es der fusionierten Unternehmen erlauben die Preise zu senken (trotz der gestiegener Marktmacht), so sollte man erwarten, dass die Gewinne der Wettbewerber darunter leiden und man daher im Falle wettbewerbsfreundlicher Fusionen einen Abwärtstrend der Aktienkurse der Wettbewerber erwarten würde. Umgekehrt würde man für den Fall, dass die Marktmachteffekte die Effizienzmotivationen dominieren, erwarten, dass die Aktienkurse der Wettbewerber steigen, da die Fusion höhere Gewinne auch für die nicht fusionierenden Unternehmen im entsprechenden Markt ermöglicht.

Eventstudien sind streng genommen nicht für Anwendungen im Rahmen von ex-post Studien geeignet, einfach deshalb, weil das zentrale Ereignis typischerweise vor der Entscheidung der Wettbewerbsbehörde stattfindet und demnach die Daten für eine Analyse schon verfügbar wären. Die Methode kann aber insbesondere zu einer Untersuchung der Korrektheit der Entscheidungen von Wettbewerbsbehörden im Falle freigegebener Fusionen herangezogen werden. Denn sie ist die einzige die es erlaubt, den Effekt der Fusion (d.h. abnormale Renditen zum Zeitpunkt der Ankündigung) vom Effekt der Entscheidung (d.h. abnormale Renditen zum Zeitpunkt der Entscheidung der Wettbewerbsbehörde) zu trennen. Durch in Bezug setzen beider Beobachtungen kann die Effektivität der Intervention abgeschätzt werden (vgl. dazu Duso et al., 2006).

Es existieren allerdings auch eine Reihe von Nachteilen der Anwendung von Ereignisstudien. Sie ist beispielsweise nicht auf jede beliebige Entscheidung anwendbar, da notwendige Bedingungen unter anderem sind, dass die betroffenen Unternehmen börsennotiert sind und einen Großteil ihres Umsatzes in den von der Fusion betroffenen Märkten tätigen. Neven und Zenger (2008, S. 486) weisen - Bezug nehmend auf die umfangreiche Literatur zu diesem Gebiet - auf einige weitere Nachteile hin. So sind Aktienmärkte in der Regel nicht informationseffizient und erfüllen somit eine zentrale Annahme von Ereignisstudien nicht. Darüber hinaus können Aktienkursreaktionen aus einer Vielzahl von Ursachen hervorgehen die entweder nichts mit dem antizipierten Anstieg der Marktmacht zu tun haben oder bereits Erwartungen über entsprechende Reaktionen der Wettbewerber, wie beispielsweise Folgefusionen, widerspiegeln und die Schlussfolgerungen von Ereignisstudien somit möglicherweise in die Irre führen.

Im Bezug auf die Interpretation der Ergebnisse von Ereignisstudien weisen Neven und Zenger (2008) darauf hin, dass eine abweichende (d.h. im Sinne der Ereignisstudie ,falsche') Entscheidung der Behörde auch damit zu tun haben kann, dass sie im Vorfeld der Entscheidung über bessere Informationen verfügt hat als der Markt (z.B. interne Dokumente der Fusionsparteien oder Einschätzungen zur Marktentwicklung von Wettbewerbern). Dies würde in letzter Konsequenz bedeuten, dass Ereignisstudien eine Wettbewerbsbehörde gerade dann kritisieren würden, wenn sie ihre Möglichkeiten effizient nutzt. Eine entsprechende Evaluation auf dieser Basis würde Anreize für die Wettbewerbsbehörde schaffen, entsprechende Informationsvorteile zukünftig nicht mehr in der gleichen Intensität zu nutzen.

Natürlich ist auch der gegenteilige Fall denkbar, nämlich dass die Informationen nicht kompetent genutzt wurden und die Entscheidungen tatsächlich falsch waren. In diesen Fällen könnten Ereignisstudien ein Instrument der Qualitätskontrolle sein. Leider zeigen die eben dargelegten Argumente aber auch, dass Ereignisstudien immer dann ein gutes Qualitätsmessinstrument sind, wenn die Behörde inkompetent ist aber ein schlechtes Instrument sofern die Behörde effizient arbeitet. Dies verdeutlicht ein zentrales generelles Problem von ex-post Studien, das Neven und Zenger (2008, S. 487) wie folgt zusammenfassen: „,... 
if the ex-post prediction differs from the ex-ante decision, is it because the decision was wrong or the ex-post assessment?”

\section{Befragungen}

Die letzte von Buccirossi et al. (2008, S. 469f.) detaillierter diskutierte Methode ist die Durchführungen von Befragungen. Dabei werden mittels Fragebogen oder Interviews strukturierte Informationen von einem repräsentativen Querschnitt der in dem betreffenden Markt anzutreffenden Akteure (u.a. Kunden und Wettbewerber) erhoben. Auf diese Weise kann eine Befragung ein geeigneter Weg sein, qualitative und qualitative Informationen über vergangene und aktuelle Entwicklungen des betreffenden Marktes zu erheben. Befragungen sind generell ein sehr flexibles Instrument und können auf alle Fragestellungen angewandt werden. Idealerweise sollten Befragungen immer zusätzlich zu anderen empirischen Methoden eingesetzt werden, da auf diese Weise eine Überprüfung und Interpretation der Ergebnisse erleichtert wird. Ex-post Evaluationen von Wettbewerbspolitik die nur auf Befragungen basieren, sind allerdings eher selten. Eine Ausnahme stellt eine Studie von PricewaterhouseCoopers (2005) dar, im Rahmen derer - im Auftrag der britischen Wettbewerbsbehörden - eine Reihe von Märkten betrachtet wurde im Anschluss an die Freigabe einer Fusion durch eine der beiden Wettbewerbsbehörden. In der Studie werden Industrieexperten befragt, ob sich nach ihrer Einschätzung die Marktentwicklung so bewahrheitet hat wie seinerzeit von der Wettbewerbsbehörde im Rahmen ihrer ex-ante Untersuchungen angenommen.

Ein zentrales Problem von Befragungen liegt in der oftmals niedrigen Beteiligungs- bzw. Rücklaufquote, da die Marktteilnehmer keine Verpflichtung haben zu antworten und oft keine hinreichenden Anreize für eine Kooperation vorhanden sind oder geschaffen werden können. Hinzu kommt, dass Antworten auf Fragen im Rahmen einer Befragung nicht die gleiche Bindungswirkung zukommt wie die letztlich durchgeführten und beobachteten Handlungen. Die Befragten könnten dazu neigen, Antworten zu geben, von denen sie glauben, dass sie der Interviewer gerne hören möchte, die aber nicht unbedingt den tatsächlichen Gegebenheiten am Besten entsprechen (sog. Response Bias). Dazu können auch auf Seiten der Interviewer durch die Art der Fragestellung Situationen entstehen, in denen die gewünschte Antwort in gewisser Weise bereits nahe gelegt wird (sog. Interviewer Bias).

Vor dem Hintergrund der Charakterisierung der zentralen Methoden einer ex-post Evaluation werden in der wettbewerbspolitischen Literatur einige generell gültige Probleme bzw. Herausforderungen einer solchen Analyse diskutiert. Sabbatini (2008, S. 496) gibt den praktischen Hinweis, dass für die Durchführung der meisten Analysen letztlich zwei Datensätze erforderlich sind. Einerseits der Datensatz aus der seinerzeitigen Untersuchung sowie andererseits ein neuen Datensatz, der die Marktentwicklungen nach der Entscheidung abbildet. Es ist nicht automatisch gewährleistet, dass beide Datensätze perfekt zusammen passen und somit die Anwendung empirischer Techniken erlauben. Weiterhin ist es der Wettbewerbsbehörde (oder gar externen Evaluierern) im Regelfall nicht möglich, die Unternehmen zur Herausgabe des ex-post Datensatzes zu zwingen. Dies mag ein zentraler Grund dafür sein, dass viele ex-post Evaluationen bereits in der Entwicklungsphase scheitern.

Neven und Zenger (2008, S. 483) stellen die grundsätzliche Frage, ob im Rahmen einer ex-post Evaluation eher freigegebene oder untersagte Fusionen untersucht werden sollten. ${ }^{25}$ Für Fälle freigegebener Fusionen spricht in diesem Zusammenhang, dass die Vergleichsalternative oftmals aus den Beobachtungen vor der Fusion abgeleitet werden kann

\footnotetext{
${ }^{25}$ Grundsätzlich ist zu vermuten, dass die Evaluation von (Fusions-) Fällen mit einem ,sample selection bias' zu kämpfen hat. Dies hat im Wesentlichen damit zu tun, dass sich manche Falltypen in bestimmten Industrien eher für solche Untersuchungen anbieten als andere Kombinationen aus Falltypen und Industrien. Die jeweilige Datenverfügbarkeit spielt dabei eine große Rolle (vgl. dazu genauer Carlton, 2009).
} 
und im Wesentlichen Preisänderungen in Folge der Fusion analysiert werden müssen. Dies erleichtert die Handhabung solcher Fälle im Vergleich zu Untersagungen, für die im Rahmen der Konstruktion einer Vergleichsalternative Informationen genutzt werden müssen, die nicht beobachtbar sind. Ein weiterer Vorteil der Analyse freigegebener Fusionen ist darin zu sehen, dass auf diese Weise Erkenntnisse darüber gewonnen werden, ob die zugrunde liegende Entscheidung der Wettbewerbsbehörde richtig war oder nicht. Ein zentrales Problem einer solchen Analyse liegt jedoch darin, dass eben die untersagten Fusionen aus der Betrachtung herausfallen. Insofern konzentriert sich die Analyse nur auf die Suche nach Fehlern 2. Art unter Vernachlässigung der Fehler 1. Art. Ceteris paribus schafft eine solche Vorgehensweise Anreize für Wettbewerbsbehörden, den Fehler 2. Art möglichst klein zu halten auf Kosten des Fehlers 1. Art. Im Ergebnis heißt dies, dass ex-post Evaluationen, die nur auf Preisänderungen freigegebener Fusionen schauen, der Behörde einen Anreiz zu einer interventionistischen Überaktivität geben der aus gesellschaftlicher Sicht unerwünscht ist.

Neven und Zenger (2008, S. 485) weisen auch auf die Bedeutung der Auswahl des Evaluationszeitpunktes hin. So kann nur in seltenen Ausnahmefällen ein Gleichlauf zwischen etwaigen Marktmachteffekten einer Fusion und der Realisierung von Effizienzen vermutet werden. Vielmehr ist zu erwarten, dass die gewonnene Marktmacht relativ schnell in Form höherer Preise ausgenutzt wird, aber die Effizienzen sich erst im Laufe der Zeit materialisieren. Insofern ist dann die Wahl des Evaluationszeitpunkts eine entscheidende Determinante der Ergebnisse der Evaluation. ${ }^{26}$

In diesem Zusammenhang ist gesondert darauf hinzuweisen, dass Preisanstiege alleine nicht unbedingt eine insgesamt sinkende Konsumentenrente bedeuten müssen, sondern auch durch eine bessere Produktqualität etc. hervorgerufen und im Hinblick auf den Gesamteffekt überkompensiert werden können. Mit anderen Worten ausgedrückt müssen steigende Preise nicht zwangsläufig die Ausnutzung erhöhter Marktmacht widerspiegeln. Generell ist in diesem Zusammenhang anzumerken, dass die Evaluationsliteratur sich im Wesentlichen auf eine Analyse der Preiseffekte konzentriert ${ }^{27}$ und die anderen kurz-, mittel- und langfristigen Einflussfaktoren auf die Konsumentenrente nicht genauer analysiert.

Neben den bislang behandelten Möglichkeiten einer Quantifizierung des Konsumentennutzens der Fusionskontrolle in einzelnen Fällen bzw. Fallbündeln ist ergänzend auf einige Möglichkeiten einer Evaluation der Fusionskontrolle einer Wettbewerbsbehörde insgesamt zu verweisen (vgl. dazu ausführlich Hüschelrath, 2009a, S. 78ff. sowie S. 144ff.). So existieren eine Reihe von Untersuchungen, die die Effektivität von Fusionsauflagen untersuchen. So wurde beispielsweise sowohl von der FTC (1999) in den USA als auch von der Europäischen Kommission (2005) die Effektivität der im Rahmen von Fusionsentschei-

\footnotetext{
${ }^{26}$ Ob eine Ausdehnung des Zeitraums zwischen Entscheidung und Evaluation wünschenswert ist, hängt zentral von der Zielsetzung der Evaluation ab. Ist das Ziel eher auf einer hohen Aggregationsebene beheimatet, so kann eine Ausdehnung der Spanne durchaus sinnvoll sein. Sollen aber einzelne Fälle bzw. Fallbündel im Hinblick auf die Leistung der Wettbewerbsbehörde untersucht werden, so ist eine solche Vorgehensweise deshalb nur beschränkt sinnvoll, da mit zunehmender Ausdehnung der Spanne verschiedenste Marktentwicklungen auftreten können, die die Behörde zum Zeitpunkt der Entscheidung gar nicht kennen konnte. Insofern wird eine ausgewogene Leistungsbewertung zu einem gewissen Grad mit wachsender Spanne zunehmend schwieriger.

${ }^{27}$ Weinberg (2008) fast insgesamt 14 Fallstudien zusammen, die sich in den vergangenen knapp zwei Jahrzehnten mit der ex-post Untersuchung der Preiseffekte von freigegebenen Fusionen befasst haben. In 11 von den 14 betrachteten Fallstudien wurden im Anschluss an die Fusion Preissteigerungen festgestellt. Methodisch wurden dabei in den meisten Studien die Änderungen in den Produktpreisen der fusionierten Unternehmen verglichen mit den Preisen einer Kontrollgruppe bestehend aus anderen Unternehmen die unter vergleichbaren Marktbedingungen operierten. Bemerkenswert ist vor allem eine Sammlung von Fallstudien in Ashenfelter und Hosken (2008), die in 4 von 5 Fallstudien Preissteigerungen infolge von Fusionen in Endverbrauchermärkten findet. Insgesamt legen die Ergebnisse der Studien somit den Schluss nahe, dass die Fusionskontrolle möglicherweise strikter durchgreifen sollte, sofern sie sich dem Ziel der Konsumentenwohlfahrtsmaximierung verpflichtet fühlt.
} 
dungen verhängten Auflagen untersucht. Während sich die FTC Studie auf strukturverändernde Auflagen konzentriert und verschiedene Probleme im Rahmen der Prozesse identifiziert, betrachtet die Europäische Kommission die Effektivität von sowohl strukturellen als auch verhaltensbeschränkenden Auflagen. Ziel der Betrachtung eines Teils der insgesamt mit Auflagen freigegebenen Fusionen war es nicht nur, herauszufinden, ob die Auflage die gewünschten Effekte erzielt hat, sondern auch ob die Auflage die Effekte so erzielt hat wie es zum Zeitpunkt der Entscheidung der Europäischen Kommission erwartet wurde. Für den Fall einer Abweichung von Erwartung und Realität wurde dann jeweils versucht, die Gründe dafür zu ermitteln.

Neben diesen behördlich initiierten und durchgeführten Studien gibt es einige wissenschaftliche Beiträge zur Effektivität von Auflagen (vgl. Duso et al., 2006, und die dort genannten Studien), der Frage, inwiefern die Fusionskontrollentscheidungen durch politische Aspekte beeinflusst werden (vgl. Aktas et al., 2006, sowie Bergman et al., 2005) sowie der Bedeutung des Abschreckungseffekts im Rahmen von Fusionskontrollentscheidungen (Eckbo, 1989, Seldeslachts et al., 2006). Methodisch basieren alle diese Studien entweder auf Ereignisstudien oder Regressionsanalysen.

\subsection{Methoden zur Abschätzung der Effekte von Kartellaufdeckungen}

Das grundlegende methodische Vorgehen bei der Quantifizierung von Kartelleffekten ist relativ einfach. Ausgehend von dem beobachteten (erhöhten) Kartellpreis muss ein so genannter ,but-for'-Preis ermittelt werden, welcher existiert hätte, sofern die Unternehmen von einer Absprache abgesehen und sich stattdessen wettbewerblich verhalten hätten. Der Unterschied zwischen dem tatsächlichen Preis und dem ,but-for'-Preis muss nun nur noch multipliziert werden mit der Ausbringungsmenge (für die Gesamtdauer des Kartells) um eine grobe Abschätzung der Wirkungen des Kartells auf die Konsumentenwohlfahrt ableiten zu können.

Obwohl diese grundlegende Technik der Quantifizierung von Kartelleffekten relativ einfach erscheint, so müssen in der praktischen Analyse oftmals eine Vielzahl an Hindernissen überwunden werden. So ist es mitunter schwierig, einen einheitlichen Marktpreis zu bestimmen, die exakte Dauer des Kartells zu ermitteln, die Weitergabe der erhöhten Einkaufspreise an nachgelagerte Wertschöpfungsstufen (sog. ,pass on' Effekte) festzustellen sowie die Berücksichtigung von Steuern in die Analyse einzupflegen. Eine besondere Herausforderung stellt jedoch regelmäßig die Ermittlung des ,but-for'-Preises dar, auch deshalb, weil die jeweiligen Endergebnisse zumeist sehr sensitiv auf kleine Änderungen des ,but-for'-Preises reagieren. Für eine fundierte Abschätzung des ,but-for'-Preises hält die ökonomische Literatur eine Vielzahl von Methoden bereit deren jeweilige Geeignetheit stark von dem vorliegenden Markt sowie den verfügbaren Daten abhängt. Eine Möglichkeit stellt beispielsweise die Verwendung von Marktpreisen dar, die vor der Bildung des Kartells im betreffenden Markt zu beobachten waren. Weiterhin ist es oftmals möglich, Vergleichsmärkte aus dem Ausland heranzuziehen um ,but-for'-Preise abzuleiten. Weiterhin können einfache homogene oder heterogene Oligopolmodelle genutzt werden, um einen wettbewerblichen Marktpreis per Simulation zu ermitteln - auch die Verwendung struktureller Modelle ist möglich. ${ }^{28}$ Darüber hinaus ist es denkbar, mit Hilfe von Kostendaten (sowie prozentualen Zuschlägen zur Gewährleistung eines branchenüblichen Rendite) 'but-for'-Preise abzuleiten (vgl. van Dijk und Verboven (2005) für eine ausführliche Beschreibung der verschiedenen Methoden).

\footnotetext{
${ }^{28}$ Im Rahmen eines homogenen Cournot-Modells lässt sich der ,but-for’ Preis - unter einer Reihe von Annahmen - mit der folgenden Formel approximieren (wobei $P$ für den ,but-for' Preis, $c$ für die Grenzkosten, $\varepsilon$ für die Marktnachfrageelastizität und HHI für den Herfindahl-Hirschman Index steht):
}

$$
\mathrm{P}=\frac{\varepsilon_{\mathrm{D}}^{\mathrm{m}} \cdot \mathrm{c}}{\left(\varepsilon_{\mathrm{D}}^{\mathrm{m}}-\mathrm{HHI}\right)} .
$$


Alle Methoden haben mit gewissen Problemen bzw. Herausforderungen zu kämpfen (siehe dazu auch die allgemeinen Erläuterungen zu den Methoden in Abschnitt 3.2.3.1.1 oben). So müssen die beobachtbaren Marktpreise vor Bildung des Kartells nicht unbedingt einem durchschnittlichen Wettbewerbspreis über einen längeren Zeitraum hinweg nahe kommen. Denn es ist beispielsweise denkbar, dass gerade durch einen Preiskrieg etc. die Kartellbildung erst ausgelöst wurde. Auch eine Betrachtung der Preise nach Kartellaufdeckung müssen nicht zwangsläufig die wettbewerblichen Preise widerspiegeln, sondern können auch im Rahmen gelernter stillschweigender Absprachen auf erhöhtem Niveau verbleiben (vgl. dazu Werden, 1998, de Roos, 2007 und Harrington, 2006). Hinzu kommt, dass die Unternehmen einen gemeinsamen Anreiz haben, die Marktpreise nach der Aufdeckung hochzuhalten um auf diese Weise zu verhindern, dass deutliche Preisrückgänge im Anschluss an die Kartellaufdeckung später im Rahmen der Prozesse gegen die Firmen verwendet werden und sich die zu zahlende Strafe bzw. der zu zahlende Schadenersatz erhöht (vgl. Harrington, 2006). Auch ein Vergleich des betreffenden Marktes mit Märkten im Ausland ist nur dann aussagekräftig, wenn die Marktbedingungen und Unternehmenscharakteristika ungefähr vergleichbar sind.

Die bislang betrachtete Ermittlung des ,but-for' Preises ist identisch zu einer Bestimmung der Schäden (Damages) in einem entsprechenden Gerichtsverfahren bzw. im Rahmen der Festlegung von Sanktionen durch die Wettbewerbsbehörde. ${ }^{29}$ In solchen Verfahren geht es allerdings naturgemäß immer um eine rückwärtsgewandte Analyse der entstandenen Schäden. Im Falle einer Untersuchung der Effekte einer Kartellaufdeckung geht es aber um die Beantwortung der vorwärtsgerichteten Frage, wie lange das Kartell noch operiert hätte (und die Konsumenten geschädigt hätte), wäre es nicht durch die Wettbewerbsbehörde aufgedeckt worden. Die somit notwendige Abschätzung einer Restlebensdauer der aufgedeckten Kartelle kann beispielsweise auf Basis historischer Durchschnittsdaten erfolgen, indem man von der durchschnittlichen Lebensdauer eines Kartells ${ }^{30}$ die Zeit in der das Kartell vor seiner Aufdeckung schon bestanden hat, abzieht. Angesichts der Tatsache, dass verschiedene Industrien aufgrund ihrer verschiedenen Charakteristika sehr unterschiedliche durchschnittliche Kartelllebensdauern (von 1 Jahr bis 100 Jahre) aufweisen (vgl. dazu Levenstein und Suslow, 2004, S. 61), erscheint zumindest eine industriespezifische Differenzierung bei der Abschätzung der Restlebensdauer sinnvoll. Idealerweise können Einschätzungen vor dem Informationshintergrund eines bestimmten Falls erfolgen.

Neben den bislang behandelten Möglichkeiten einer Quantifizierung des Konsumentennutzens von Kartellaufdeckungen in einzelnen Fällen bzw. Fallbündeln ist ergänzend auf einige Möglichkeiten einer Evaluation der Kartellverfolgung einer Wettbewerbsbehörde insgesamt zu verweisen (vgl. dazu ausführlich Hüschelrath, 2009a, S. 134ff.). Grundsätzlich muss sich dabei mit dem Problem auseinandergesetzt werden, dass sich aus der simplen Beobachtung der Anzahl an aufgedeckten Kartellen einer Wettbewerbsbehörde pro Zeiteinheit nur schwer Rückschlüsse auf die Effektivität und Effizienz der Kartellverfolgung ziehen lassen. So kann eine niedrige Anzahl an aufgedeckten Kartellen einerseits für eine erfolgreiche Abschreckungspolitik sprechen, sie kann aber auch lediglich eine mangelhafte Arbeit in der Wettbewerbsbehörde bzw. mangelnde Ressourcen widerspiegeln.

\footnotetext{
${ }^{29}$ Die Ermittlung des ,but-for' Preises für Zwecke der Evaluation muss nicht zwangsläufig die gleiche Genauigkeit aufweisen wie dies im Rahmen der Schadensberechnung erforderlich ist. Dies liegt daran, dass es bei der Schadensberechnung (zumindest in dem entsprechenden privatwirtschaftlichen Verfahren) zentral um die Frage geht, wer wie viel Schaden durch das Kartell davongetragen hat, während der Fokus im Rahmen der Evaluation eher auf der Schadenshöhe insgesamt liegt (die dann in die Zukunft fortgeschrieben wird). Daraus folgt beispielsweise, dass im Rahmen einer Evaluation der Erfassung des Weitergabeeffekts der Preiserhöhung an nachfolgende Stufen (sog. pass-on Effekt) eine geringere Bedeutung zukommt, als dies im Falle der konkreten Schadensermittlung für eine bestimmte Wertschöpfungsstufe der Fall wäre.

${ }^{30}$ Connor und Zimmermann (2005) errechnen beispielsweise mit Hilfe eines Datensatzes von 204 internationalen Kartellen die im Zeitraum von 1990 bis 2004 aufgedeckt wurden, dass die durchschnittliche Kartelllebensdauer in diesem Sample bei 6,3 Jahren lag.
} 
Infolgedessen kann ein Anstieg in der Anzahl an aufgedeckten Kartellen einerseits als Erfolg etwaiger Reformen des wettbewerbspolitischen Instrumentariums gesehen werden, es ist aber auch denkbar, dass die Anzahl der Kartelle in einer Volkswirtschaft angestiegen ist und die Wettbewerbsbehörde - trotz eines suboptimalen Kartellverfolgungskonzepts - mit größerer Wahrscheinlichkeit das eine oder andere Kartell entdeckt.

Aus diesem Identifikationsproblem lässt sich direkt der Wunsch nach Hilfsindikatoren für eine Beurteilung der Kartellverfolgung ableiten. Eine generelle qualitative Möglichkeit besteht beispielsweise in der Definition einer Best Practice der Kartellverfolgung und einer anschließenden Überprüfung, inwieweit diese Best Practice in der betreffenden Wettbewerbsbehörde umgesetzt ist (vgl. dazu Hüschelrath, 2009a, S. 199ff.). So ließe sich beispielsweise aus der Feststellung, dass eine Kartellbehörde keine Kronzeugenregelung implementiert hat oder kaum mit anderen Wettbewerbsbehörden kooperiert ableiten, dass dann auch das (nicht direkt beobachtbare) Ergebnis der Kartellverfolgung nicht optimal sein kann. Ein weiterer Hilfsindikator besteht in einem Vergleich der verhängten Strafen zu den theoretisch optimalen Strafen (d.h. diejenige Strafe, die den Abschreckungseffekt optimiert). Findet eine entsprechende Analyse, dass die tatsächlichen Strafen regelmäßig und deutlich unter den optimalen liegen, so kann auf eine suboptimale Kartellverfolgung geschlossen werden (vgl. dazu Veljanovski, 2007). Eine letzte hier anzusprechende Möglichkeit der ex-post Evaluation besteht in einer Analyse der entsprechenden Märkte nach der Aufdeckung des Kartells. Wird hier systematisch festgestellt, dass sich beispielsweise die Preise kurze Zeit nach der Aufdeckung wieder dem Preisniveau während der Absprache nähern, so ist es schwierig, einen dauerhaften Effekt der aktuellen Kartellverfolgung zu identifizieren (vgl. u.a. Feinberg, 1984, Connor (1998), de Roos (2007) sowie Harrington, 2006). Gründe hierfür können in den - von der Aufdeckung unbeeinflussten - kollusionsfreundlichen Marktstrukturen und den sich daraus ergebenden Möglichkeiten einer expliziten oder stillschweigenden Kollusion gesehen werden. ${ }^{31}$

\subsubsection{Gesamte Wettbewerbspolitik als Evaluationsgegenstand}

Im Anschluss an eine Betrachtung der Indikatoren und Methoden im Rahmen einer Evaluation von Fällen bzw. Fallbündeln soll in den folgenden Abschnitten der Aggregationsgrad erhöht werden und die gesamte Wettbewerbspolitik eines Landes als Evaluationsgegenstand betrachtet werden. Dabei erweist sich eine Gliederung in zwei größere Abschnitte als zweckmäßig. So werden im Folgenden zunächst praktische Methoden von Wettbewerbsbehörden bzw. Unternehmensberatungen vorgestellt, gefolgt von einem Überblick über einige wissenschaftliche Versuche einer Evaluation von Wettbewerbspolitik.

\subsection{Methodologische Vorgehensweisen in der Praxis}

Die wohl wesentlichste Zielsetzung einer ex-post Evaluation der gesamten Wettbewerbspolitik in der Praxis ist in der Rechenschaftsablegung zu sehen. Wie im Folgenden zu verdeutlichen sein wird, sind die im Rahmen solch ganzheitlicher Evaluationen verwendeten Methoden derart grob, dass sie sich nur beschränkt zur Erzielung von Erkenntnisgewinnen eignen.

In gewisser Weise die einzige Ausnahme dieser einleitenden Einschätzung stellt ein expost Evaluationsinstrument dar, welches einerseits fallbasiert arbeitet und andererseits aber auf fortlaufender Basis eine umfassende Evaluation der Wettbewerbspolitik vornimmt: Die gerichtlichen Überprüfungen der Entscheide der Wettbewerbsbehörden (vgl. dazu insbesondere Bergman, 2008, S. 389f.). Das Recht einer gerichtlichen Überprüfung steht jeder

\footnotetext{
${ }^{31}$ Neben Preisen können grundsätzlich auch andere Parameter wie beispielsweise die Angebotsstruktur (insbes. Markteintritte) oder die Nachfragestruktur untersucht werden im Hinblick auf mögliche Effekte der Kartellaufdeckung (vgl. u.a. Hüschelrath et al., 2009 sowie die generellen Erläuterungen im obigen Fusionsteil).
} 
Unternehmung (oder gegebenenfalls auch jedem Individuum) zu im Falle einer unvorteilhaften Entscheidung der Wettbewerbsbehörde. Ein Gericht nimmt dann eine erneute Einschätzung der Sachlage vor und überprüft die Entscheidung der Wettbewerbsbehörde im Stile einer ex-post Evaluation.

Basierend auf diesen Erläuterungen wäre ein nahe liegendes Maß für eine ex-post Evaluation die simple Betrachtung der Erfolgsquote der Entscheide der Wettbewerbsbehörde vor Gericht anzusehen. ${ }^{32}$ Leider identifiziert Bergman (2008, S. 389f.) einige Gründe, weshalb dieser Indikator nur auf den ersten Blick besonders geeignet erscheint. So werden beispielsweise nicht alle potentiell falschen Entscheidungen gleichermaßen von den Unternehmen angefochten. Im Fall von Untersagungen von Fusionsvorhaben können beispielsweise langwierige Klagen die erwarteten Vorteile der Fusion derart reduzieren, dass die Unternehmen nach einem negativen Entscheid der Wettbewerbsbehörde von ihrem Ansinnen ablassen. Daneben kann eine Klage in der Regel auch nur von benachteiligten Parteien eingereicht werden, das heißt dann im Umkehrschluss, dass Entscheidungen der Wettbewerbsbehörde bestimmte Fälle nicht zu untersuchen, nicht angefochten werden können. Eine weitere Einschränkung der Nutzung von Gerichtsentscheiden als Evaluationsinstrument ist, dass im Rahmen solcher Untersuchungen die juristischen Argumente im Vordergrund stehen - diese müssen sich nicht automatisch vollständig überschneiden mit den volkswirtschaftlich optimalen Entscheidungen.

Im Hinblick auf die Anreizwirkung von Evaluationen ist zu bedenken, dass Wettbewerbsbehörden die nur nach der Erfolgsrate vor Gericht beurteilt werden einen Anreiz hätten, eher kleinere Fälle mit schwachen Parteien zu betrachten da von deren Seite Klagen unwahrscheinlicher sind. Im Umkehrschluss ist es demnach fragwürdig, ob es ein Ziel der Wettbewerbsbehörde sein sollte, die Erfolgsquote zu maximieren. Auch länderübergreifende Vergleiche sind in diesem Zusammenhang nur schwer möglich aufgrund der bestehenden Unterschiede in den Rechtssystemen. Darüber hinaus ist unklar, wie eine hohe Erfolgsrate zu deuten wäre da sie sowohl eine starke Performance der Behörde nahe legen kann aber auch die Tatsache widerspiegeln kann, dass die Wettbewerbsbehörde sehr defensiv agiert. Hilfreich können allerdings Beobachtungen der Veränderungen der Erfolgsquoten innerhalb einer Jurisdiktion sein. So war ein Abfall in der Erfolgsquote eine zentrale Ursache für die jüngsten Reformen innerhalb der EU Kommission - insbesondere die neue Fusionskontrollverordnung sowie Änderungen in der internen Organisationsstruktur (u.a. die Einführung des Postens eines Chefökonomen). Hintergrund war der abweichende gerichtliche Entscheid im Falle dreier bedeutender Fusionsentscheidungen der Europäischen Kommission (jeweils mit der Begründung einer mangelhaften bzw. nicht ausreichend belegten ökonomischen Argumentation).

Neben der Betrachtung der Erfolgquote vor Gericht gibt es vielfältige weitere Möglichkeiten einer direkten Messung des (vordergründigen) Outputs von Wettbewerbsbehörden. So ließen sich beispielsweise die Anzahl an Verfahren und Entscheiden, die Verfahrensdauern, die Anzahlen an publizierten Bekanntmachungen oder auch die Intensität des Informationsaustauschs mit anderen Wettbewerbsbehörden als Outputindikatoren deuten. Wie allerdings bereits im Falle der Einschätzungen zur Kartellpolitik oben verdeutlicht wurde, sind solche Indikatoren oftmals schwer zu interpretieren und bestenfalls dann mit einer gewissen Aussagekraft verbunden, wenn sie über die Zeit hinweg für eine Behörde erhoben werden und dann grundlegende Verschiebungen beobachtet werden können (vgl. dazu Saurer, 2008, S. 20f. am Beispiel der Schweiz). In den Worten Kovacics (2008, S. 131) ausgedrückt: „To say that an agency is doing a lot of things or only a few things does not tell us whether it is doing the right things. In sport, coaches admonish athletes not to

\footnotetext{
${ }^{32}$ Daraus lässt sich - Bezug nehmend auf Bergman (2008) - ableiten, dass zusätzliche Ressourcen in ex-post Evaluationen von Bereichen investiert werden sollten, die nicht regelmäßigen gerichtlichen Überprüfungen unterliegen.
} 
equate activity with accomplishment.” Aus diesem Grund soll im Rahmen dieses Beitrags auf eine genauere Diskussion solcher Outputindikatoren verzichtet werden.

Neben der Erstellung solcher einfachen Outputstatistiken der Arbeit von Wettbewerbsbehörden existieren einige weitere regelmäßige qualitative Evaluationen der gesamten Wettbewerbspolitik. So werden beispielsweise von der OECD regelmäßige Expertenüberprüfungen (sog. ,peer reviews') organisiert im Rahmen derer die gesamte Wettbewerbspolitik einzelner Staaten evaluiert wird und darauf aufbauend Verbesserungsvorschläge unterbreitet werden (vgl. dazu z.B. OECD, 2008, am Beispiel der Ukraine). Darüber hinaus konstruiert die OECD beispielsweise einen ,Competition Law and Policy Indicator' für alle OECD Mitgliedsstaaten mit dem das Ziel verfolgt wird, zwei zentrale Politiken zur Förderung des Wettbewerbs - Wettbewerbspolitik und Deregulierungspolitik - in einem Indikator abzubilden und länderübergreifend vergleichbar zu machen (vgl. Høj et al., 2007).

Neben der OECD sind auch privatwirtschaftliche Unternehmen im Bereich der Evaluation von Wettbewerbspolitik aktiv wie beispielsweise das Global Competition Review (GCR), das jedes Jahr eine Studie veröffentlicht in denen die einzelnen Wettbewerbsbehörden umfassend evaluiert und abschließend in eine Rangfolge gebracht werden. Die für die Bewertung notwendigen Informationen werden aus einem Mix aus Experteneinschätzungen und Kundenzufriedenheitsumfragen (inbes. bei Kartellrechtsanwälten) gewonnen. Teilweise greifen auch jüngere akademische Studien auf die vom GCR gesammelten Daten zurück mit dem Ziel der Herstellung einer Vergleichbarkeit von verschiedenen Wettbewerbspolitiken (vgl. dazu insbesondere Nicholson 2007, 2008). Allen diesen Studien ist gemein, dass sie für eine Quantifizierung der Effekte von Wettbewerbspolitik kaum hilfreich sind und auch nur beschränkte Erkenntnisgewinne für die einzelnen Teilbereiche der Wettbewerbspolitik versprechen. Sie erlauben aber auf hoher Aggregationsebene einen internationalen Leistungsvergleich und können so etwaig verfügbare Evaluationsressourcen in die erfolgträchtigsten Bereiche lenken.

Neben Gerichten, staatlichen sowie privaten Organisationen unterziehen sich seit einiger Zeit auch einige Wettbewerbsbehörden einer Selbstevaluation ihrer Aktivitäten. So nimmt beispielsweise das Office of Fair Trading (2008a) in Großbritannien - vor dem Hintergrund einer entsprechenden Vorgabe seitens des Finanzministeriums - seit einigen Jahren eine Quantifizierung des Nutzens seiner Aktivitäten für die Verbraucher vor und stellt diesen dem bereitgestellten Budget gegenüber. Die niederländische Wettbewerbsbehörde (NMa) ist vor kurzem sogar dazu übergegangen, die positiven Auswirkungen ihrer Aktivitäten auf makroökonomische Faktoren wie Wachstum und Beschäftigung zu messen (vgl. dazu Don et al., 2008). Im Vergleich zu diesen beiden relativ neuen Evaluationsansätzen müssen die Vereinigten Staaten als routiniert im Bereich der Evaluation von Wettbewerbspolitik bezeichnet werden, da dort bereits seit 1993 der so genannte Government Performance and Results Act (GPRA) vorschreibt, die ungefähren Auswirkungen der Aktivitäten der beiden Wettbewerbsbehörden mit Hilfe geeigneter Leistungsindikatoren zu dokumentieren (vgl. dazu Werden, 2008). In allen Fällen wird dabei ein so genannter bottom-up Ansatz verfolgt; das bedeutet, dass von der individuellen Fallentscheidung ausgegangen wird und mit Hilfe von Daumenregeln bzw. einfachen Simulationsprogrammen eine grobe Abschätzung der Konsumenteneffekte erfolgt. Diese Daten werden anschließend aggregiert im Rahmen der Teilbereiche (wie beispielsweise Fusionskontrolle oder Kartellverfolgung) und schließlich über die gesamte Behörde hinweg. In einem letzten Schritt erfolgt dann ein Vergleich des ermittelten Gesamtnutzens mit einer Abschätzung der Kosten. Im Folgenden sollen die Evaluationskonzepte des DOJ und des OFT - aus Gründen der gebotenen Knappheit jeweils nur für die Bereiche Fusionen und Kartelle - skizziert werden.

Das Evaluationskonzept des US Department of Justice (DOJ) verfolgt das Ziel einer Abschätzung der Ersparnisse der Konsumenten durch die behördlichen Aktivitäten in den 
Bereichen Fusionskontrolle, Kartellverfolgung und Marktmachtmissbrauch. ${ }^{33}$ Im Bereich der Fusionskontrolle wird eine grobe Abschätzung der Konsumenteneinsparungen fallweise abgeleitet, indem man die jährlichen Umsätze im relevanten Markt multipliziert mit dem wahrscheinlichen Preisanstieg der eingetreten wäre, wenn die Wettbewerbsbehörde die Fusion nicht verboten oder mit Auflagen versehen hätte (vgl. Werden, 2008, S. 447). Die Abschätzungen der Preiserhöhungen erfolgen dann mit dem jeweils passenden Fusionssimulationsprogramm. In Falle des Vorliegens von Fallentscheidungen mit differenzierten Produkten in Endverbrauchermärkten wird ein Fusionssimulationstool auf Basis eines differenzierten Bertrand-Modells angewandt. Für alle anderen Fusionen werden die Einsparungen mit Hilfe eines Fusionssimulationstools auf Basis eines Cournot-Modells abgeleitet. Als Dateninput für letztgenanntes Modell werden lediglich Marktanteile, die Marktnachfrageelastizität vor der Fusion sowie Informationen über die Kosten benötigt. Allerdings sind dabei - unter Hinweis auf die obigen Erläuterungen zu Simulationen - eine Reihe von Annahmen unterstellt.

Im Falle der Abschätzung der Konsumenteneinsparungen durch die Verfolgung von Kartellen wendet das DOJ eine einfache Regel an (vgl. Werden, 2008, S. 446). Für Kartelle mit einer Lebensdauer von 1 Jahr oder länger werden einmalig die Ersparnisse der Konsumenten mit 10\% der jährlichen Umsätze im relevanten Markt angenommen. Für Kartelle mit einer Dauer von unter einem Jahr wird die Abschätzung von 10\% aller Umsätze im relevanten Markt für die Dauer des Kartells angesetzt. Der 10\%-Wert wird begründet als defensive Schätzung des durchschnittlichen Preiseffekts eines Kartells. Vor dem Hintergrund der in der ökonomischen Literatur vielfach dokumentierten tatsächlichen durchschnittlichen Preissteigerungen ${ }^{34}$ muss dieser Wert als eher konservativ angesehen werden. Für eine Unterschätzung der Effekt entspricht auch, dass die Zeitspanne der Konsumenteneinsparungen durch die Kartellaufdeckung auf 1 Jahr begrenzt ist, obwohl es sehr wahrscheinlich ist, dass viele Kartelle ohne Aufdeckung wohl noch deutlich länger operiert hätten. Auch hierfür existieren - wie oben bereits angedeutet - verschiedene empirische Belege für verschiedene Industrien.

Das Office of Fair Trading (OFT) in Großbritannien veröffentlicht seit dem Geschäftsjahr 2005/06 unter dem Titel ,Positive Impact' eine Quantifizierung seiner Aktivitäten im Hinblick auf die Konsumentenwohlfahrt (vgl. OFT, 2005, 2007a, 2008a). Dabei werden nicht nur Fusionen und Kartelle in die Analyse miteinbezogen, sondern auch andere Bereiche der Wettbewerbspolitik und des Konsumentenschutzes. Die grundlegende Vorgehensweise im Hinblick auf die Quantifizierung der Effekte unterscheidet sich nicht wesentlich von der eben skizzierten Konzeption des DOJ. Das OFT hat allerdings seit der Einführung der Evaluation 2005/2006 sowohl das Evaluationskonzept als auch die Evaluationsmethoden kontinuierlich verbessert und verfeinert. So nutzt das OFT in seiner neuesten Studie beispielsweise nicht mehr pauschal einen Preiseffekt von 1\% für eine untersagte bzw. mit Auflagen genehmigte Fusion, sondern verwendet wenn möglich Fusionssimulationsprogramme für deren Ermittlung (vgl. genauer OFT, 2007b, für eine detaillierte Beschreibung). Ferner wurde die Wirkungsdauer der Preiseffekte nach Fusionen von einem auf zwei Jahre verlängert. Im Bereich der Kartellverfolgung wurde die Bestimmung der hypothetischen Restlebensdauer von Kartellen (ohne entsprechende Intervention) auf Basis eigener Erhebungen neu geregelt (vgl. OFT, 2007a, S. 32). Im Falle von Kartellen, die zum Zeitpunkt der Aufdeckung sieben Jahre oder weniger installiert waren, wird von einer noch durch-

\footnotetext{
${ }^{33}$ Von einer Darstellung des Evaluationsansatzes im Bereich des Marktmachtmissbrauchs wird hier abgesehen und auf Werden (2008) verwiesen.

${ }^{34}$ Connor und Lande (2006, S. 1004) geben beispielsweise einen Überblick über sechs verschiedene quantitative Studien, die eine Abschätzung der durchschnittlichen Preiserhöhungen durch Kartelle (unter Nutzung unterschiedlicher Datensätze) vornehmen. Demnach liegt die durchschnittliche Preiserhöhung über alle Studien hinweg bei 28,1\% (ungewichteter Median), die Streuung der Werte von 7,8\% bis 44,5\% ist allerdings sehr beachtlich.
} 
schnittlich zu erwartete Restlebensdauer von sechs Jahren ausgegangen. Im Falle von Kartellen von über sieben Jahre Laufzeit zum Zeitpunkt der Aufdeckung wird die durchschnittliche Restlebensdauer abgeschätzt durch die Multiplikation der aktuellen Lebensdauer mit dem Faktor 1,4 und der anschließenden Subtraktion von dreieinhalb Jahren.

Vor dem Hintergrund der kurzen Beschreibungen der Evaluationskonzepte von OFT und DOJ lässt sich insgesamt feststellen, dass beide Konzepte einige methodologische Probleme aufweisen und demnach ihre Aussagekraft beschränkt ist. So gehen beide Messkonzepte beispielsweise davon aus, dass die Entscheidungen der Wettbewerbsbehörde immer richtig waren. Wenngleich diese Annahme im Falle von Kartellen in der Regel zutreffend ist, so kann davon im Falle anderer Wettbewerbsbeschränkungen nicht automatisch ausgegangen werden. Ein weiteres zentrales Problem stellt die mangelnde Berücksichtigung des Abschreckungseffekts dar. In diesem Zusammenhang wird vielfach betont, dass dies der wichtigste Effekt wettbewerbspolitischer Aktivitäten überhaupt sei. Ist dies der Fall, so muss man gleichzeitig die Aussagekraft der Ergebnisse vorliegender Evaluationen anzweifeln, da diese keine Messung dieses Effekts vornehmen. Ein weiterer Kritikpunkt liegt in der unzureichenden Erfassung der Kosten von Wettbewerbspolitik. Hierunter sind keinesfalls nur die staatlichen Aufwendungen für die Wettbewerbsbehörde zu summieren, sondern beispielsweise ebenso die Compliance- und Verfahrensaufwendungen der Unternehmen sowie Kosten, die durch die Abschreckung eigentlich wettbewerbskonformer Verhaltensweisen der Gesellschaft entstehen (vgl. Tabelle 1 in Abschnitt 3.2.1 oben für eine Übersicht der Kostenkategorien). Eine weitere auffällige methodologische Schwäche liegt in der ausschließlichen Betrachtung der Preiseffekte unter Missachtung möglicher anderer Quellen von Konsumentennutzen bzw. durch das Kartell verursachter Schäden. Dies gilt insbesondere auch für vom Kartell verursachte produktive Ineffizienzen (u.a. Rent Seeking Ausgaben, X-Ineffizienzen, Kosten der Aufrechterhaltung des Kartells) sowie etwaige dynamische Ineffizienzen (u.a. Erschwerung von Markteintritt, Rückgang der Innovationsdynamik) und den damit ausgelösten Wirkungen auf die Konsumentenwohlfahrt. Hinzu kommt, dass die beschriebenen Methoden als relativ grob bezeichnet werden müssen und nur schwer abzuschätzen ist, ob die Analysen eher zu einer Unter- oder Überschätzung der Nutzen führen. Darauf weisen auch die zum Teil bestehenden Unterschiede in den Annahmen der Analyse hin (u.a. im Hinblick auf die durchschnittlich angenommene Kartelldauer oder den durchschnittlichen Preisanstieg bei Fusionen). ${ }^{35}$ Im Hinblick auf die Quantifizierung der Kartelleffekte gibt es mindestens vier weitere methodologische Kritikpunkte. So stellt das oben charakterisierte Verfahren der Ermittlung der Konsumentenvorteile nur eine grobe Näherung dar, da es einerseits den entsprechenden Deadweight loss nicht mit in die Analyse einbezieht sowie andererseits die Auswirkungen des Kartells auf die Preise unbeteiligter Firmen (sog. Umbrella-Effekte) nicht betrachtet. Der erstgenannte Punkt würde für eine Unterschätzung der tatsächlichen Vorteile sprechen, während dies beim zweiten nicht pauschal eingeschätzt werden kann, sondern einer genaueren Analyse bedarf. Weiterhin ist unklar, ob die recht langen unterstellten Restlebensdauern der aufgedeckten Kartelle wirklich einen guten Durchschnittswert darstellen. Ein Gegenargument zu dieser Sichtweise besagt, dass Wettbewerbsbehörden überwiegend die ineffizienten und ohnehin kurz vor der Auflösung stehenden Kartelle aufdecken und die wirklich erfolgreichen unentdeckt bleiben (vgl. Shughart und Tollison, 1998). Sollte dies tatsächlich so der Fall sein, dann würde dies für eine Überschätzung der Konsumenteneffekte durch das beschriebene

\footnotetext{
${ }^{35}$ Bergman (2008) untersucht die Plausibilität der Annahme, dass Kartelleffekte viel länger andauern als Fusionseffekte. In beiden Fällen ist das zentrale Argument für die Beendigung der Effekte stattfindender Markteintritt. In diesem Zusammenhang zweifelt Bergman an, dass Markteintritt nach einer Fusion wirklich systematisch schneller stattfindet als nach einem Kartell. Eine starke Allianz der fusionierten Parteien mag viel bessere Möglichkeiten haben, Wettbewerber von einem Markteintritt abzuschrecken und hat zudem keine Probleme mit Abweichlern etc. Ein Gegenargument wäre freilich, dass eintretende Firmen vom Kartell aufgenommen werden könnten und es somit weiterexistiert.
} 
Verfahren sprechen. Das gleiche Resultat würde sich ergeben, wenn die Marktpreise nach der Kartellaufdeckung zwar idealerweise kurzfristig auf das Wettbewerbsniveau (den ermittelten ,but-for' Preis) fallen, aber danach relativ zügig wieder auf ein Niveau oberhalb des wettbewerblichen Preises ansteigen. Auch in einem solchen Fall würden die Quantifizierungen den Effekt auf die Konsumentenwohlfahrt überschätzen. ${ }^{36}$ Vor dem Hintergrund dieser Vielzahl an Problemen gehen einige Kommentatoren (vgl. Neven und Zenger, 2008) sogar so weit, zu fragen, ob es überhaupt sinnvoll ist, unter diesen Umständen Ressourcen in solche sehr grobschlächtigen Untersuchungen zu investieren.

Eine jüngere Studie von Deloitte (2007) im Auftrag des OFT versucht in diesem Zusammenhang, ein methodologisches Problem - die Bedeutung des Abschreckungseffekts einer Lösung näher zu bringen. Dabei lag das Hauptziel der Studie direkt in der Ermittlung des Umfangs des Abschreckungseffekts (im Verhältnis zu den direkten Effekten) in den Bereichen Fusionen, Kartellen und missbräuchlichen Verhaltensweisen. Dazu wurden zwischen Mai und November 200630 Interviews mit Juristen, Ökonomen und Unternehmen geführt. Zusätzlich wurden zwei Telefonumfragen durchgeführt - die Erste bei insgesamt 234 Anwälten für Wettbewerbsrecht und die Zweite bei insgesamt 202 Unternehmen in Großbritannien mit mehr als 200 Mitarbeitern. Im Rahmen des umfassenden Fragenkatalogs wurde der Abschreckungseffekt im Falle von Fusionen gemessen als Anzahl der Fusionen die im Anschluss an die Einholung von externem anwaltlichen Rat nicht weiterverfolgt wurden oder verändert wurden bevor das OFT Kenntnis von ihnen erlangte relativ zur Anzahl der Fusionen die im Zuge einer OFT Intervention untersagt oder modifiziert wurden. Die Ergebnisse zeigen für die Periode von 2000 bis 2006 ein Verhältnis von 5 zu 1 für die Fusionskontrolle und die Kartellverfolgung sowie von $10 \mathrm{zu} 1$ für missbräuchliche Verhaltensweisen - jeweils das Verhältnis von Abschreckungseffekt zu direkten Effekten abbildend. Gordon und Squires (2008, S. 418) sehen diese Schätzungen noch als defensiv an, da die Studie ausschließlich die Fusionen beinhaltet, die im Anschluss an die Nutzung externer anwaltlicher Beratungsdienstleistungen abgebrochen oder modifiziert wurden. In der Praxis wird aber mit zunehmender Größe der Unternehmung eine zunehmende Tendenz festzustellen sein, dass solche Überlegungen intern - d.h. ohne Hinzuziehung von externen Anwälten - getroffen werden. Die parallel durchgeführte Unternehmensumfrage bestätigt diese Sichtweise insofern, als sie zu dem Ergebnis kommt, dass durchschnittlich nur jede vierte Fusion externen Anwälten zur Beurteilung übertragen wird.

\subsection{Methodologische Vorgehensweisen in der Wissenschaft}

Neben den im vorangegangenen Abschnitt beschriebenen praktischen Versuchen einer Evaluation von Wettbewerbspolitik gibt es auch von wissenschaftlicher Seite zahlreiche Versuche, die positiven Effekte von Wettbewerbspolitik auf die jeweilige Volkswirtschaft messbar zu machen. Im Folgenden sollen daher einige methodologische Ansätze kurz skizziert werden. Dabei wird weder ein Anspruch auf Vollständigkeit erhoben, noch wird auf die Ergebnisse der Studien eingegangen.

Die wohl ältesten Studien, die sich im weiteren Sinne einer Evaluation von Wettbewerbspolitik zurechnen lassen, stellen Abschätzungen der Bedeutung der NettoWohlfahrtsverluste für Industrien bzw. ganze Volkswirtschaften dar. Wie Gleichung (3) verdeutlicht, hängt die Größe des Netto-Wohlfahrtsverlusts $D W L$ ab von der Preis-Kosten Marge $M$, den Industrieumsätzen $R$ sowie der Preiselastizität der Marktnachfrage $\varepsilon$

$$
D W L=\frac{1}{2} M^{2} \cdot R^{M} \cdot \varepsilon_{D}
$$

Harberger (1954) sowie einige andere Wissenschaftler nach ihm versuchten nun eine Abschätzung der Höhe des Netto-Wohlfahrtsverlusts unter Verwendung durchschnittlicher

\footnotetext{
${ }^{36}$ Eine geeignete Reaktion der Wettbewerbsbehörde auf diese Problematik könnte darin bestehen, die betreffenden Märkte auch nach der Entscheidung zu beobachten um auf diese Weise die Wahrscheinlichkeit erneuter Absprachen zu reduzieren.
} 
Preis-Kosten Margen und durchschnittlicher Nachfrageelastizitäten. Motiviert von den am Anfang überraschend kleinen Effekten fanden dann in der Folgezeit Ausweitungen der Studien statt indem neben den Netto-Wohlfahrtsverlusten auch Rent-Seeking Ausgaben und produktive Ineffizienzen in die Analysen mit aufgenommen wurden (vgl. Hüschelrath, 2009a, S. 12ff.für einen Überblick). Abgesehen von einer Vielzahl an methodischen Problemen dieser Studien ist ein zentraler inhaltlicher Kritikpunkt, dass zum Herausrechnen des Effekts der Wettbewerbspolitik eine Vergleichsalternative ohne eine solche Politik konstruiert werden muss. Da dies nicht möglich ist, verdeutlichen die Studien im Wesentlichen nur, dass es selbst für den Fall, dass Wettbewerbspolitik nur einen prozentual minimalen Einfluss hat, die positiven Wohlfahrtswirkungen dennoch enorm sein können (vgl. Bergman, 2008).

Stigler (1966) kann als Pionier einer Studienart angesehen werden, die Vergleiche anstellt zwischen einem Ausgangsland bzw. Industrien und a) anderen Ländern die keine Wettbewerbsgesetzgebung aufweisen, b) zwischen Perioden bevor bzw. nachdem bestimmte Gesetze wirksam wurden und c) zwischen Industrien, die vom geltenden Wettbewerbsrecht ausgenommen wurden. Unter Nutzung dieser Untersuchungsstrategien zeigt beispielsweise Warzynski (2001) mit Hilfe eines Datensatz des produzierenden Gewerbes in den USA, dass die Preis-Kosten-Margen in Phasen einer schärferen Verfolgung von Wettbewerbsverstößen niedriger waren als in Phasen, die eher mit einer ,Politik der langen Leine' der beiden Wettbewerbsbehörden umschrieben werden können. Konings et al. (2001) vergleichen - ebenso für das produzierende Gewerbe - die Entwicklungen der Preis-Kosten Margen in den Niederlanden und Belgien und nutzten dabei die Tastsache aus, dass Belgien bereits im Jahre 1993 eine Reform der Wettbewerbsgesetze vornahm, die Niederlande aber erst 1998. Die Ergebnisse zeigen, dass zwar keine signifikanten Effekte der Reform auf die Preis-Kosten Margen in Belgien gefunden werden konnten, aber dafür die Preis-Kosten Margen in den Niederlanden signifikant höher waren als in Belgien. Symeonidis (2002) unternimmt einen Vergleich der Entwicklung von Industrien in Großbritannien die zum Zeitpunkt der Einführung des Kartellverbots kartelliert bzw. nicht kartelliert waren und findet, dass der Wettbewerb in den kartellierten Industrien zunahm und zu einem Absinken der Preis-Kosten Margen führte. Als letztes Beispiel sei auf eine Studie von Clarke and Evenett (2003, S. 725f.) verwiesen, die für den Fall des internationalen Vitamin-Kartells zu dem Ergebnis kommen, dass die von den Unternehmen verlangten Preisaufschläge in Staaten mit einer scharfen Verfolgung von Wettbewerbsverstößen geringer waren als in Staaten mit einer eher großzügigen Auslegung des Wettbewerbsrechts. Dahinter steckt der Gedanke, dass Unternehmen in Ländern mit scharfer Verfolgung von Wettbewerbsverstößen die Preisaufschläge geringer wählen, um auf diese Weise die Aufdeckungswahrscheinlichkeit zu reduzieren.

Geleitet von der Überzeugung, dass auch der Effekt von Wettbewerbspolitik auf makroökonomische Größen einen messbaren Effekt hat, simulieren van Sinderen und Kemp (2008, S. 376ff.) den Effekt von Wettbewerbspolitik auf Wachstum des BIP, Produktivität $^{37}$ und Beschäftigungswachstum. Eine Auswertung bestehender Studien zu diesem Gebiet zeigt, dass alle betrachteten Studien einen positiven Zusammenhang von mehr Wettbewerb auf Wachstum und Beschäftigung finden. Die meisten Studien zeigen darüber hinaus auch einen Anstieg der Produktivität. Ermutigt von diesen Ergebnissen verdeutlichen die Autoren zunächst das Konzept der statischen Nutzenbestimmung und gehen dann dazu über, die Effekte auf die makroökonomischen Variablen abzuschätzen (vgl. van Sinderen und Kemp, S. 378ff.). Sie nutzen dafür ein Simulationsprogramm welches die dynami-

\footnotetext{
${ }^{37}$ In einer noch andauernden Untersuchung betrachten Buccirossi et al. (2009) den Zusammenhang zwischen der Effektivität der Wettbewerbspolitik und dem Wachstum der totalen Faktorproduktivität. Basierend auf einem selbst konstruierten Indikatorensystem und Daten für 22 Industrien in 12 OECD Staaten von 1995 bis 2005 zeigen die vorläufigen Ergebnisse, dass Wettbewerbspolitik einen stark positiven Effekt auf das Wachstum der totalen Faktorproduktivität ausübt.
} 
schen Aspekte von Marktgleichgewichten in mittel- und langfristiger Perspektive abbilden kann. Es ist dadurch in der Lage, die makroökonomischen Effekte mikroökonomischer Eingriffe zu simulieren. Dabei nehmen die Autoren an, dass ein Marktmachtanstieg letztlich wie eine Steuererhöhung wirkt und entsprechend modelliert werden kann. Die Ergebnisse der Simulation zeigen für den Zeitraum von 1998 bis 2007 einen positiven Effekt auf die Produktion in der Größe von 0,5\% sowie einen positiven Effekt auf die Beschäftigung in Höhe von $0,4 \%{ }^{38}$

\subsection{Ergebnisse, Würdigung und Schlussfolgerungen}

Die Auswertungsstufe einer Politikevaluation besteht aus drei zentralen Bausteinen: Der Ableitung und Interpretation der Ergebnisse, der Würdigung der Aussagekraft sowie der Ableitung von Schlussfolgerungen aus den Evaluationsergebnissen. Diese im zweiten Kapitel allgemein erläuterten Bausteine werden im Folgenden in Bezug gesetzt zu bestehenden Arbeiten im Bereich der Wettbewerbspolitik.

\subsubsection{Ableitung und Interpretation der Ergebnisse}

Im Anschluss an die Anwendung der für die jeweilige Fragegestellung als geeignet befundenen Methoden muss die Ableitung und Interpretation der Ergebnisse erfolgen. In Abhängigkeit des Evaluationsobjekts, der Zielsetzung der Evaluation sowie der angewandten Methoden kann es nicht nur notwendig sein, Einzelergebnisse zu interpretieren und in den Kontext zu stellen, sondern es kann zunächst auch erforderlich sein, Aggregationen von Einzelergebnissen zu einem Gesamtergebnis vorzunehmen. So sind beispielsweise im Rahmen der oben skizzierten Versuche einer Evaluation der gesamten Wettbewerbspolitik mit der Ableitung der Teilergebnisse zwar alle notwendigen Daten erhoben, es muss allerdings noch eine Zusammenführung und gegebenenfalls der beabsichtigte Vergleich von Gesamtkosten zu Gesamtnutzen erfolgen. Im Rahmen einer solchen Aggregation kann dann möglicherweise auch eine Gewichtung von Einzelkriterien bzw. -ergebnissen vorgenommen werden.

Neben der Ableitung der Ergebnisse ist im Anschluss eine Interpretation derselben vorzunehmen. In diesem Zusammenhang sollte der Versuch unternommen werden, die zentralen Treiber der Ergebnisse zu diskutieren und gegebenenfalls auch klar begründete Verbesserungsvorschläge zu entwickeln. Ist dies aufgrund des hohen Aggregationsgrades der Untersuchung nicht oder nur beschränkt möglich, so bleibt gegebenenfalls noch die Identifikation von Problembereichen die dann in der Folge genauer untersucht werden könnten. Grundsätzlich auch dem Bereich der Interpretation der Ergebnisse zuzuordnen ist die Würdigung von Problemen mit der vorliegenden Untersuchung wie beispielsweise der fehlende Einbezug von zentralen anderen Determinanten der Konsumentenrente (außerhalb des Marktpreises) wie Produktqualität, Produktvielfalt oder die unklare Bedeutung von Abschreckungseffekten. Im Falle der hier vorgeschlagenen Methodologie sollen diese Aspekte allerdings separat im folgenden Baustein behandelt werden. Grundsätzlich gilt auch auf der Auswertungsebene der Grundsatz der anderen beiden Ebenen, nämlich dass im Regelfall eine simultane und keine iterative Betrachtung der einzelnen Bausteine innerhalb der jeweiligen Ebene geboten ist.

Ein letzter Bereich, der dem vorliegenden Baustein zuzuordnen ist, ist die Frage nach der Kommunikation der Ergebnisse. Interne Studien die nicht veröffentlicht werden, entziehen sich einerseits der Überprüfung durch die Öffentlichkeit, andererseits muss im Rahmen der Kommunikation der Ergebnisse auch bedacht werden, dass diese nicht nur externe Wirkungen entfalten, sondern auch interne Wirkungen auf die Anreizstrukturen in

\footnotetext{
${ }^{38}$ Im Hinblick auf die Höhe der Effekte muss allerdings bemerkt werden, dass vor 1998 Kartelle in den Niederlanden nur genehmigungspflichtig waren. Insofern kann daraus geschlossen werden, dass die Einführung von Wettbewerbsrecht einen überdurchschnittlich großen Anstoßeffekt hat, der nicht unbedingt dauerhaft so zu erwarten und beobachten sein wird.
} 
der Behörde sowie die Arbeitsmotivationen der Mitarbeiter haben können (vgl. dazu Neven und Zenger, 2008 sowie der nachfolgende Abschnitt). Hinzu kommt, dass die Veröffentlichung der Ergebnisse gewisser Studienarten bzw. -ergebnisse auch rechtliche Probleme nach sich ziehen könnte. Dies trifft insbesondere auf Studien zu, in denen die Wettbewerbsbehörde selbst Fehler einräumt. In solchen Fällen kann es durchaus sinnvoll sein, die Ergebnisse einer Evaluation der Öffentlichkeit nicht zugänglich zu machen. Die gleiche Schlussfolgerung gilt vermutlich für Evaluationen mit einem sehr unvorteilhaften Ergebnis für die Wettbewerbsbehörde. Im Regelfall stellt allerdings eine Veröffentlichung der Vorgehensweise und Ergebnisse der Evaluation eine gute Gelegenheit dar, die Qualität der Vorgehensweise zu überprüfen und gleichzeitig die Diskussionen um Verbesserungsmöglichkeiten zu stimulieren.

\subsubsection{Würdigung der Aussagekraft}

Mit diesem Baustein soll das zentrale Ziel verfolgt werden, die spezifische Vorgehensweise der Evaluation vor dem Hintergrund der erzielten Ergebnisse abschließend zu würdigen und insbesondere ihre Schwächen klar zu benennen. Dazu zählt es unter anderem, an allen kritischen Stellen auf bedeutsame Annahmen sowie methodische Probleme hinzuweisen und idealer Weise begründete Vermutungen im Hinblick auf die Auswirkungen auf die Ergebnisse abzuleiten. Im Rahmen einer solchen Untersuchung kann es im quantitativen Teil sinnvoll sein, Sensitivitätsüberprüfungen durchzuführen, also der Frage nachzugehen, wie schnell und in welcher Deutlichkeit sich die Ergebnisse durch die Abänderung bestimmter Annahmen ändern.

Ohne an dieser Stelle auf alle im Rahmen der vergangenen Abschnitte identifizierten methodologischen und/oder methodischen Probleme eingehen zu wollen, so soll eine kurze Zusammenfassung einiger zentraler Problemherde ausgewählter Bausteine erfolgen. So wurde im Rahmen der Identifikation des Evaluationsgegenstandes festgestellt, dass eine klare Festlegung desselben - verbunden mit der Formulierung einer Forschungsfrage eine wesentliche Voraussetzung für eine erfolgreiche Evaluation ist. Dies gilt in besonderem Masse für die Verknüpfung des Evaluationsgegenstands mit den Zielen, die die Evaluation verfolgen soll. Hier sollten insbesondere die Gründe für die vorgenommene Auswahl und Priorisierung der Ziele erläutert werden. In diesen Bereich fällt ebenso eine Einschätzung zu möglichen Problemen mit der gewählten Art der Untersuchung. Je nachdem ob sich für eine interne oder externe Umsetzung entschieden wurde, können andere Probleme im Laufe der Evaluation entstanden sein, die Auswirkungen auf die Aussagekraft der Ergebnisse haben. So spielt beispielsweise mangelnde Datenverfügbarkeit bei Externen eine potentiell große Rolle, während bei Internen Befangenheit und mangelnde Methodenkenntnis die Aussagekraft der Ergebnisse einschränken können. Auch eine Würdigung des Kontexts, d.h. des Umfelds der Analyse im Hinblick auf Rahmen und Ausgangsbedingungen, kann hilfreich sein um die Aussagekraft der Ergebnisse besser einschätzen zu können. Gleiches gilt für die Auswahl des Untersuchungszeitpunkts für den unter anderem aufgezeigt werden sollte, ob die Ergebnisse als reagibel im Hinblick auf eine Änderung desselben einzuschätzen sind.

Neben den Zielen der Evaluation sind die Ziele des Evaluationsobjekts sowie das daraus abgeleitete Kriterienset von großer Bedeutung für eine Einschätzung der Wirkung der Aussagekraft. So ist es einerseits für den Erfolg der Evaluation zwingend, die Ziele möglichst genau zu definieren, andererseits wurde im Bezug auf die wettbewerbspolitische Praxis festgestellt, dass die genauen Zielsetzungen im Regelfall nicht so eindeutig definiert sind, dass sie ohne weitere Diskussion in einer Evaluation angewandt werden könnten. Insofern sollte im Rahmen einer Würdigung der Ergebnisse auf mögliche Probleme und deren mögliche Wirkungen auf das Ergebnis eingegangen werden. Gleiches gilt für die unmittelbar in Zusammenhang stehende Frage nach der Auswahl der Kriterien und Indikatoren. In diesem Zusammenhang wurde in Tabelle 1 in Abschnitt 3.2.1 oben eine ganze Tabelle möglicher 
Kriterien präsentiert, die sich im Regelfall nicht alle im Rahmen einer Studie in Indikatoren übertragen und messen lassen. Insofern muss im Rahmen dieses Bausteins nicht nur eine Begründung für die Auswahl bestimmter Kriterien und Indikatoren erfolgen, sondern sofern möglich auch eine Einschätzung darüber gegeben werden, wie der Einbezug anderer Indikatoren die Ergebnisse beeinflussen könnte. Einschätzungen dynamischer Komponenten sowie bestehende Interaktionen bzw. Überlappungen zwischen Politiken bzw. Maßnahmen sind ebenso zu berücksichtigen - dies gilt in gleichem Maße für die Einschätzungen zum Baustein ,Festlegung von Vergleichsalternativen’.

Im Rahmen der Auswahl und Anwendung der Evaluationsmethoden gibt es sicherlich den größten Spielraum in der Vorgehensweise und somit auch den größten Erklärungsbedarf. So ist detailliert zu begründen, weswegen eine bestimmte Auswahl von Fällen für einen bestimmten Untersuchungsabschnitt ausgewählt wurde und inwiefern man diesen Ausschnitt als ,repräsentativ für die Grundgesamtheit' betrachten kann. Hinzu kommen eine Vielzahl an fusions- und kartellspezifischen methodischen Problemen die in Bezug gesetzt und in ihren Wirkungen eingeschätzt werden müssen (wie beispielsweise die Vernachlässigung der Netto-Wohlfahrtsverluste sowie die Annahmen bzgl. der Preissteigerungen oder der Wirkungsdauern der Kartell- bzw. Fusionseffekte, vgl. dazu die vorangegangenen Abschnitte 3.2.3.1 und 3.2.3.2).

Insgesamt betrachtet ist es im Rahmen von Wirksamkeits- sowie vor allem bei Wirtschaftlichkeitsanalysen besonders wichtig, eine detaillierte Einschätzung darüber vorzunehmen, welche Kosten- und Nutzenkomponenten gar nicht bzw. nur qualitativ in die Analyse eingegangen sind und ob diese Nichtbetrachtung eher zu einer Unter- oder Überschätzung der Kosten bzw. Nutzen führt. Im Hinblick auf die Unterschätzung der Nutzen muss beispielsweise - Bezug nehmend auf die obigen Erläuterungen sowie auch van Sinderen und Kemp (2008, S. 378) - auf die Probleme hingewiesen werden, die rein statische Betrachtungen der Wohlfahrtseffekte von wettbewerbspolitischen Eingriffen beinhalten. Beschränken sich Wirkungsanalysen beispielsweise auf den betreffenden Sektor in welchem sich der Fall zugetragen hat, so werden positive (aber gegebenenfalls auch negative) Spillover-Effekte auf andere Unternehmen und/oder andere Märkte ausgeblendet.

Ein weiterer Aspekt, der im Regelfall mit einer Unterschätzung des Nutzens in Verbindung gebracht wird, ist der Abschreckungseffekt. In Anlehnung an die detaillierten Ausführungen von Gordon und Squires (2008, S. 412) fließen im Regelfall nur die direkten Effekte in die Analysen ein, nicht aber der Nutzen der aufgrund aktiver Wettbewerbspolitik durch das Unterlassen von Wettbewerbsverstößen generiert wird.

Als Gegenspieler einer Nutzenunter- bzw. Kostenüberschätzung sind im Sinne einer ausgewogenen Evaluation auch Aspekte zu berücksichtigen, die eher für eine Nutzenüberbzw. Kostenunterschätzung sprechen könnten. So ist es in Anlehnung an das obige Argument von van Sinderen und Kemp $(2008,378)$ auch denkbar, dass in den Nutzenabschätzungen nicht adäquat berücksichtigt wird, dass Konsumenten im Falle von Preiserhöhungen möglicherweise zu anderen Produkten wechseln und so folglich den Wohlfahrtsgewinn von Wettbewerbspolitik reduzieren. Darüber hinaus ist es denkbar, dass Wettbewerbseingriffe der Wettbewerbsbehörden mittel- und langfristig den Konsumenten schaden. Ein mögliches Beispiel wäre, dass es in einer bestimmten Phase des Produktlebenszyklus zu einer marktmächtigen Position kommt, diese aber in der Folge auch ohne wettbewerbspolitische Intervention wegkonkurriert worden wäre und die vorübergehenden Monopolrenten wiederum wichtig gewesen wären zur Deckung getätigter F\&E Investitionen und somit zur Aufrechterhaltung der Innovationsanreize. Generell verdeutlicht dieses Beispiel, dass Evaluationen selten dynamische Effekte abbilden können und somit regelmäßig mit derartigen Argumenten angreifbar sind.

Ein weiterer Faktor, der sowohl die Nutzen reduzieren als auch die Kosten von Wettbewerbspolitik erhöhen kann sind fehlerhafte Entscheidungen der Wettbewerbsbehörden. Die in einem Großteil der Studien anzutreffende Annahme, dass alle Entscheidungen der Wett- 
bewerbsbehörde richtig waren, ist in der Praxis vermutlich nicht zutreffend. ${ }^{39}$ Die Kosten falscher Entscheidungen sind dabei keineswegs nur auf den betreffenden Fall beschränkt, sondern von ihnen geht eine Signalwirkung an alle Unternehmen aus, die die Nutzen von Wettbewerbspolitik insgesamt substantiell reduzieren kann. So zeigen Schinkel und Tuinstra (2006), dass eine Kombination aus fehlerhaften Entscheidungen und Interventionsfreude auf Seiten der Wettbewerbsbehörde die Wahrscheinlichkeit für Wettbewerbsverstöße sogar erhöht. Dies liegt einerseits daran, dass es mit steigender Wahrscheinlichkeit für einen Fehler 2. Art immer unwahrscheinlicher wird, dass die Wettbewerbsverstöße erkannt werden. Andererseits gilt, dass mit steigender Wahrscheinlichkeit eines Fehlers 1. Art es immer wahrscheinlicher wird, dass eine Unternehmung zu Unrecht bestraft wird. Beide Argumente erhöhen den Anreiz, Wettbewerbsverstöße vorzunehmen.

Ein weiteres zentrales Argument bezieht sich auf eine Unterschätzung der Kosten. In diesem Zusammenhang ist generell zu bemerken, dass es im Hinblick auf eine ausgewogene Evaluation der Wettbewerbspolitik nicht ausreichend ist, nur die Nutzenkomponenten mit immer höherer Genauigkeit zu messen und anschließend einfach dem Budget der Wettbewerbsbehörde gegenüberzustellen. Durch ein solches Vorgehen bleiben vielfältige Kostenkomponenten unberücksichtigt. So gehen beispielsweise ComplianceAufwendungen nur selten in Analysen auf der Kostenseite ein. Gleiches gilt beispielsweise für Gerichtskosten. Ebenso ist es grundsätzlich notwendig, die Gefahr negativer Abschreckungseffekte einzuschätzen. Diese können entstehen, wenn durch Unklarheiten in der Rechtsanwendung bzw. den anfallenden Durchsetzungskosten sich Unternehmen entscheiden, wettbewerbsrechtlich eigentlich unbedenkliche Strategien bzw. Produkte nicht auf den Markt zu bringen. Ferner ist im Zusammenhang mit der Erfassung der Kosten zu überlegen, ob nicht auch die Kosten nach wettbewerbspolitischen Teilbereichen aufgeschlüsselt werden sollten um somit Kosten-Nutzen Vergleiche auf Basis von Teilgebieten zu ermöglichen. Dies wäre ganz im Sinne der Forderung Kovacics (2006, S. 596ff.) nach einer genaueren Untersuchung der Implementierungskosten von Wettbewerbspolitik und der damit zusammenhängenden Frage, wie behördeninterne Prozesse vereinfacht und damit kostengünstiger gestaltet werden können.

\subsubsection{Schlussfolgerungen aus den Evaluationsergebnissen}

Der letzte Schritt einer Evaluation sollte dazu dienen, auf Basis der Ergebnisse Lern- und Implementationsprozesse zu starten und gegebenenfalls Konsequenzen zu ziehen. Wie in Kapitel 2 bereits erläutert, hängen die jeweiligen Schlussfolgerungen dabei sowohl von den Zielen der Evaluation als auch vom Evaluationstyp ab. Im Folgenden sollen einige zentrale Aspekte im Zusammenhang mit der Evaluation von Wettbewerbspolitik angesprochen werden.

Eine zentrale Bedeutung im Hinblick auf die Konsequenzen einer Evaluation ist in den Auswirkungen auf das Verhalten der Wettbewerbsbehörde zu sehen. So wurde bereits an verschiedenen Stellen darauf hingewiesen, dass suboptimal ausgestaltete Evaluationen nicht nur die individuellen Arbeitsmotivationen der behördlichen Mitarbeiter verändern können, sondern auch der Behördenleitung Anreize setzt, Ressourcen innerhalb der Behörde volkswirtschaftlich suboptimal umzuschichten, zu interventionistisch zu agieren oder relevante Informationen zu ignorieren (vgl. Neven und Zenger, 2008). Werden Behörden beispielsweise nur noch nach dem messbaren Beitrag zur Konsumentenwohlfahrt evaluiert, so haben sie naturgemäß einen Anreiz - um den Nutzenzuwachs möglichst groß ausfallen

\footnotetext{
${ }^{39}$ In diesem Zusammenhang sei allerdings daran erinnert, dass die Fehleranfälligkeit wettbewerbspolitischer Aktivitäten asymmetrisch über die Teilbereiche verteilt ist. Ein Bereich mit geringer Anfälligkeit ist die Kartellverfolgung, da zumeist das Vorliegen eines Kartells unstrittig ist und die negativen Wohlfahrtswirkungen vorausgesetzt werden und nicht belegt werden müssen. Für alle anderen Bereiche der Wettbewerbspolitik ist die Fehleranfälligkeit größer einzuschätzen, da die Wohlfahrtswirkungen untersucht werden müssen und die Wahrscheinlichkeit einer Fehlentscheidung somit ansteigt.
} 
zu lassen - große Fälle zu betrachten unter Vernachlässigung der kleineren Märkte. Ein solches Vorgehen kann in der Folge durchaus negative Auswirkungen auf die Aufrechterhaltung des Abschreckungseffekts haben, da beispielsweise die Verfolgung kleinerer Kartelle durchaus eine wichtige Signalfunktion haben kann (vgl. Niels und van Dijk, 2008, S. 361).

Ein weiteres zu behandelndes Problem im Rahmen dieses Bausteins ist die Frage, wie häufig eine Evaluation durchgeführt bzw. wiederholt werden sollte. So wurde oben beschrieben, dass einige Wettbewerbsbehörden jedes Jahr umfassend über die Kosten und Nutzen ihrer Aktivitäten berichten. Hier wäre die Frage zu stellen, ob dies aus ökonomischer Sicht sinnvoll ist oder ob nicht eine Evaluation in größeren Zeitabständen vorzuziehen ist. Neven und Zenger (2008, S. 488) bemerken in diesem Zusammenhang, dass nur sehr beschränkte Ressourcen für solche umfassenden Studien investiert werden sollten. Da die Nutzenüberschüsse in allen Studien derart deutlich sind, erscheint es wenig sinnvoll, durch verbesserte Methoden einen kleineren Genauigkeitsgewinn zu verzeichnen. Bergman (2008) fügt hinzu, dass aufgrund des Ausschlusses des Abschreckungseffekts im Rahmen der Quantifizierung sich automatisch die Frage anschließen muss, welchen tieferen Sinn Quantifizierungen haben, wenn die potentiell größte Nutzenkomponente sich einer Erfassung entzieht. Vor diesem Hintergrund vermutet Bergman (2008) - in Verbindung mit entsprechenden Anregungen der OECD (2005, S. 10) - einen potentiell größeren Erkenntnisgewinn durch die Schaffung einheitlicher Methoden und Standards, die es in der Folge ermöglichen würden, Wettbewerbsbehörden international besser zu vergleichen. Solche Vergleiche hätten ein wirkliches Potential die jeweiligen Best Practices zu definieren und die eigenen Standards als Wettbewerbsbehörde daran zu orientieren.

Die vermutlich wichtigste Frage innerhalb dieses Bausteins ist jedoch, wie die Ergebnisse der Evaluation konkret genutzt werden sollen. So legen Buccirossi et al. (2008, S. 472) als auch Bergman (2008) besonderen Wert auf die Feststellung, dass es im Rahmen einer ex-post Evaluation nicht nur darum gehen kann, den Effekt der Entscheidung auf die Konsumentenwohlfahrt zu isolieren, sondern vor allem auch der Wettbewerbsbehörde zu helfen, ihre Entscheidungsprozesse zu verbessern. Dazu sind nicht nur Informationen notwendig, ob eine Entscheidung richtig war oder nicht, sondern auch weshalb sie so entstanden ist. Kovacic (2006) weist vor diesem Hintergrund sehr deutlich auf die große Bedeutung der Prozessanalyse hin. Insofern kann ein zentrales Ergebnis der im Rahmen dieses Beitrags im Wesentlichen vorgestellten Wirksamkeits- und Wirtschaftlichkeitsanalysen durchaus die Einleitung eines oder mehrerer Projekte mit dem Ziel einer Prozessverbesserung sein. Solch eine Prozessevaluation „....might seek to assess the quality of the competition agency's internal operations - the mix of managerial methods and organizational choices that determine how the agency allocates and applies its resources. This approach treats management and organization as critical inputs into the implementation of competition policy and seeks to identify improvements in how the competition agency operates. The logic is that progress toward superior managerial and organizational techniques will increase the likelihood that the agency's substantive outputs generally promote the realization of the competition law's objectives” (Kovacic, 2006, S. 506).

\section{Zusammenfassung und Schlussfolgerungen}

Im Jahre 2005 fand im Rahmen eines OECD Policy Roundtables ein intensiver Meinungsaustausch der Mitgliedsstaaten zu dem Thema ,Evaluation of the Actions and Resources of the Competition Authorities' statt. Eine wesentliche Erkenntnis der Gespräche bestand in der Feststellung, dass „,[c]onsiderable work remains to be done to refine the methodologies used to evaluate the effectiveness of completed competition policy interventions" (OECD, 2005,. S. 10). Obwohl seit der Formulierung dieser Arbeitsvorgabe erst einige Jahre vergangen sind, so hat die Bedeutung der Evaluation von Wettbewerbspolitik seitdem deutlich zugenommen - sowohl im wissenschaftlichen Bereich als auch im Bereich der Umsetzung 
in der Praxis. Die Schwerpunkte der Arbeiten lagen dabei allerdings nicht so sehr auf einer methodologischen Strukturierung und Aufbereitung der gesamten Problemstellung, sondern eher in der Verfeinerung bzw. Überprüfung bestimmter Methoden, der Betrachtung ausgewählter Fallstudien sowie der Umsetzung staatlicher Vorgaben der Qualitätskontrolle bzw. Rechenschaftspflichten.

Vor diesem Hintergrund verfolgte dieser Beitrag nicht nur das Ziel einer Identifikation und Charakterisierung genereller methodologischer Bausteine einer Politikevaluation, sondern er beabsichtigte vor allem eine Einordnung der Erkenntnisse bestehender wettbewerbspolitischer Arbeiten in den geschaffenen methodologischen Rahmen vorzunehmen. Diese Vorgehensweise erlaubte nicht nur eine systematische Darstellung der methodologischen Überlegungen bestehender Studien, sondern ermöglichte gleichzeitig eine Identifikation von Problemen bei der Evaluation von Wettbewerbspolitik und somit die Ableitung von Forschungsbedarf. Anstatt die wesentlichen Erkenntnisse des Beitrags an dieser Stelle nochmals in einfacher Textform wiederzugeben, liefert die folgende Tabelle 3 eine zusammenfassende Charakterisierung der methodologischen Bausteine einer Evaluation zweier bedeutender wettbewerbspolitischer Gegenstände: Fälle bzw. Fallbündel sowie die gesamte Wettbewerbspolitik. 
Tabelle 3: Charakterisierung der methodologischen Bausteine einer Evaluation zweier wettbewerbspolitischer Gegenstände

\begin{tabular}{|c|c|c|}
\hline & $\begin{array}{l}\text { Evaluationsgegenstand: } \\
\text { Fälle bzw. Fallbündel }\end{array}$ & $\begin{array}{l}\text { Evaluationsgegenstand: } \\
\text { Gesamte Wettbewerbspolitik }\end{array}$ \\
\hline \multicolumn{3}{|l|}{ I. Vorbereitung } \\
\hline $\begin{array}{l}\text { Funktionen, Ziele } \\
\text { und Kontext }\end{array}$ & $\begin{array}{l}\text { - Funktionen: Erkenntnis-, Kontroll- und } \\
\text { Dialogfunktion } \\
\text { - Ziele: Qualitätskontrolle und Identifi- } \\
\text { kation von Verbesserungspotential, Über- } \\
\text { prüfung der Wirksamkeit } \\
\text { - Kontext: Wirtschaftliches und politisches } \\
\text { Umfeld, Entwicklungsstand der Wettbe- } \\
\text { werbspolitik }\end{array}$ & $\begin{array}{l}\text { - Funktionen: Legitimations- und Dialog- } \\
\text { funktion } \\
\text { - Ziele: Nachkommen einer Rechenschafts- } \\
\text { pflicht, Überprüfung der Wirtschaft- } \\
\text { lichkeit } \\
\text { - Kontext: Wirtschaftliches und politisches } \\
\text { Umfeld, Entwicklungsstand der Wettbe- } \\
\text { werbspolitik }\end{array}$ \\
\hline $\begin{array}{l}\text { Auswahl des Eva- } \\
\text { luations-zeitpunkts }\end{array}$ & $\begin{array}{l}\text { - Ex-post Analyse oder begleitende Analy- } \\
\text { se }\end{array}$ & - Begleitende Analyse \\
\hline \multicolumn{3}{|l|}{ II. Durchführung } \\
\hline $\begin{array}{l}\text { Auswahl eines } \\
\text { Kriteriensets }\end{array}$ & $\begin{array}{l}\text { - Grundannahme: Ziel der Konsumenten- } \\
\text { wohlfahrtsmaximierung } \\
\text { - Berücksichtigung des Einflusses auf den } \\
\text { Preis sowie andere Mikrovariablen (z.B. } \\
\text { Qualität, Auswahl sowie dynamische As- } \\
\text { pekte) }\end{array}$ & $\begin{array}{l}\text { - Grundannahme: Ziel der Konsumenten- } \\
\text { wohlfahrtsmaximierung } \\
\text { - Berücksichtigung der aggregierten Effek- } \\
\text { te auf Mikro- und Makrovariablen (z.B. } \\
\text { Produktivität, Beschäftigung) } \\
\text { - Berücksichtigung indirekter Kosten sowie } \\
\text { sozialer Kosten }\end{array}$ \\
\hline $\begin{array}{l}\text { Festlegung von } \\
\text { Vergleichs- } \\
\text { alternativen }\end{array}$ & $\begin{array}{l}\text { - Vergleichsalternativen bei Fusionen hän- } \\
\text { gen ab von der Entscheidung der Behörde } \\
\text { und dem Verhalten der Fusionsparteien } \\
\text { - Vergleichsalternative bei Kartellen ist der } \\
\text { Markt ohne Absprache (vorwärtsgewandt) }\end{array}$ & $\begin{array}{l}\text { - Vergleichsalternativen sind ,keine Wett- } \\
\text { bewerbspolitik', ,wettbewerbsrechtliche } \\
\text { Reformen' oder, nur privatwirt- } \\
\text { schaftliche Durchsetzung des Wettbe- } \\
\text { werbsrechts' }\end{array}$ \\
\hline $\begin{array}{l}\text { Auswahl und An- } \\
\text { wendung von } \\
\text { Indikatoren und } \\
\text { Methoden }\end{array}$ & $\begin{array}{l}\text { - Zentrale Indikatoren: Marktpreis, Trans- } \\
\text { aktionsvolumen, Qualität, Markt-struktur- } \\
\text { und Marktverhalten } \\
\text { - Methoden: Simulationen, Strukturelle } \\
\text { Modelle, Natürliche Experimente und } \\
\text { Matching-Methoden, Ereignisstudien, } \\
\text { Befragungen }\end{array}$ & $\begin{array}{l}\text { - Indikatoren: Marktpreis, Transaktions- } \\
\text { volumen, Marktstruktur- und Marktver- } \\
\text { halten, makroökonomische Variablen, } \\
\text { direkt messbare Outputs der Behörde } \\
\text { - Methoden: Daumenregeln, einfache Si- } \\
\text { mulationen und Befragungen (Praxis) } \\
\text { sowie ökonometrische Verfahren und Si- } \\
\text { mulationen (Wissenschaft) }\end{array}$ \\
\hline \multicolumn{3}{|l|}{ III. Auswertung } \\
\hline $\begin{array}{l}\text { Ableitung und } \\
\text { Interpretation der } \\
\text { Ergebnisse }\end{array}$ & $\begin{array}{l}\text { - Zusammenführung und Interpretation } \\
\text { - Ableitung von Schlussfolgerungen und } \\
\text { Verbesserungsbedarf } \\
\text { - Kommunikationsform der Ergebnisse }\end{array}$ & $\begin{array}{l}\text { - Aggregation der Einzelnutzen zu einem } \\
\text { Gesamtnutzen } \\
\text { - Vergleich mit den Kosten } \\
\text { - Ableitung von Schlussfolgerungen } \\
\text { - Kommunikationsform der Ergebnisse }\end{array}$ \\
\hline $\begin{array}{l}\text { Würdigung der } \\
\text { Aussagekraft }\end{array}$ & $\begin{array}{l}\text { - Generell: Einschätzung möglicher Analy- } \\
\text { seschwächen und deren Wirkung auf die } \\
\text { Ergebnisse } \\
\text { - Speziell: Probleme rein statischer Analy- } \\
\text { sen, Vernachlässigung von Netto- } \\
\text { Wohlfahrtsverlusten und Spillover- } \\
\text { Effekten, Methodische Schwächen durch } \\
\text { Probleme der Datenverfügbarkeit bzw. } \\
\text { andere Restriktionen, Verzerrungen durch } \\
\text { Fallauswahl bzw. Evaluationsteam }\end{array}$ & $\begin{array}{l}\text { - Generell: Einschätzung möglicher Analy- } \\
\text { seschwächen und deren Wirkung auf die } \\
\text { Ergebnisse } \\
\text { - Speziell: Probleme rein statischer Analy- } \\
\text { sen, Vernachlässigung von Abschre- } \\
\text { ckungs- und Spillover-Effekten, Kosten } \\
\text { durch fehlerhafte Ent-scheidungen, Me- } \\
\text { thodische Schwächen bei der Nutzenab- } \\
\text { schätzung, Unzureichende Erfassung der } \\
\text { Kostenseite }\end{array}$ \\
\hline $\begin{array}{l}\text { Schlussfolger- } \\
\text { ungen aus den } \\
\text { Evaluations- } \\
\text { ergebnissen }\end{array}$ & $\begin{array}{l}\text { - Anstoß von Lern- bzw. Implementati- } \\
\text { onsprozessen (insbes. Prozessevaluatio- } \\
\text { nen) } \\
\text { - Einflüsse auf die Anreize der Mitarbeiter } \\
\text { und der Leitung der Behörde } \\
\text { - Wiederholungszyklus der Evaluation }\end{array}$ & $\begin{array}{l}\text { - Überarbeitung des Regelwerks bzw. der } \\
\text { Politiken und/oder deren Umsetzung } \\
\text { - Budgeterweiterungen bzw. -kürzungen } \\
\text { - Einflüsse auf die Anreize der Mitarbeiter } \\
\text { und der Leitung der Behörde } \\
\text { - Wiederholungszyklus der Evaluation }\end{array}$ \\
\hline
\end{tabular}


Generell lässt sich abschließend sagen, dass Wirksamkeitsanalysen derzeit die größte Bedeutung in Wissenschaft wie Praxis haben und andere Evaluationsziele wie die Untersuchung der Wirtschaftlichkeit oder die Prozessverbesserung eine deutlich geringere Rolle zu spielen scheinen. In den beiden letztgenannten Feldern schlummert infolgedessen noch umfangreiches ungenutztes Forschungspotential. Dies gilt umso mehr als - wie in diesem Beitrag gezeigt wurde - insbesondere Wirksamkeitsanalysen und Untersuchungen mit dem Fokus auf Prozessverbesserungen eher in einer komplementären als in einer substitutiven Beziehung zueinander stehen.

Eine ebenso große Fülle an ungenutztem Forschungspotential gibt es - zumindest im Vergleich zu anderen Disziplinen wie der Arbeitsmarkt- oder Innovationspolitik - im Bereich der Weiterentwicklung und Verfeinerung der Evaluationsmethoden. Eine notwendige technische Voraussetzung für Forschungsfortschritte in diesem Bereich ist es allerdings, Zugang zu detaillierten Datenbeständen entweder von Seiten der Unternehmen oder von Seiten der Wettbewerbsbehörden zu ermöglichen. Ohne die Erfüllung dieser Vorbedingung wird es die Evaluation der Wettbewerbspolitik schwer haben, sich methodisch weiterzuentwickeln und sich folglich dem Niveau anderen Disziplinen anzunähern. Diese Forderung wird abschließend auch von Dennis Carlton (2009, S. 21) - zumindest für den Bereich der Fusionskontrolle in den USA - so bekräftigt: "Antitrust analysis of individual cases has gotten increasingly sophisticated. Evaluation of antitrust policy has not. There is a need to gather post merger industry data and a need to gather the predictions of DOJ merger analysis in order to evaluate whether U.S. policy and analysis can be improved. Strong opinions are not substitutes for quantitative analysis." Vor diesem Hintergrund erscheinen die Forderungen von William Kovacic (2009, S. 144) nach einem intensiveren und institutionalisierten Austausch zwischen wettbewerbspolitischer Praxis und wettbewerbspolitischer Forschung nur allzu berechtigt.

\section{Literaturverzeichnis}

Aktas, N., E. De Bodt, und R. Roll (2006), European M\&A Control is Protectionist, Working Paper, Anderson Graduate School of Management, University of California, Los Angeles.

Ashenfelter, O. und D. Hosken (2008), The Effect of Mergers on Consumer Prices: Evidence from Five Selected Case Studies, NBER Working Paper, Cambridge.

Bergman, M. (2008), Quis Custodiet Ipsos Custodes? or Measuring and Evaluating the Effectiveness of Competition Enforcement, De Economist, Vol. 156, 387-409.

Bergman, M., M. Jakobsson, und C. Razo (2005), An Econometric Analysis of the European Commission's Merger Decisions, International Journal of Industrial Organization, Vol. 23, 717-737.

Besanko, D. und D. Spulber (1993), Contested Mergers and Equilibrium Antitrust Policy, Journal of Law, Economics, \& Organization Vol. 9, 1-29.

Buccirossi, P., Ciari, L., Duso, T., Spagnolo, G. und C. Vitale (2009), Competition Policy and Productivity Growth: An Empirical Assessment, Working Paper, Rome.

Buccirossi, P., Ciari, L., Duso, T., Fridolfsson, S.-O., Spagnolo, G. und C. Vitale (2008), A Short Overview of a Methodology for the Ex-Post Review of Merger Control Decisions, De Economist, Vol. 156, 453-475.

Bundesamt für Justiz (1999), Wirksamkeitsüberprüfung/Evaluation, Bern.

Bundesministerium für Wirtschaft und Arbeit (2004), Evaluation staatlicher Interventionen aus Auftraggebersicht, Schlussbericht der Projektgruppe „Evaluation“, Berlin.

Bussmann, W. (1995), Evaluationen staatlicher Maßnahmen erfolgreich begleiten und nutzen: Ein Leitfaden, Bern.

Bussmann, W., Klöti, U. und P. Knoepfel (1997), Einführung in die Politikevaluation, Basel. 
Carlton, D. (2009), Why We Need to Measure the Effect of Merger Policy and How to Do It, NBER Working Paper, Cambridge.

Clarke, J. und S. Evenett (2003), The Deterrent Effects of National Anticartel Laws: Evidence from the International Vitamins Cartel, Antitrust Bulletin, Vol. 48, 689-726.

Competition Commission (2008), Evaluation of the Competition Commission's Past Cases, London.

Connor, J. und J. Zimmerman (2005), Determinants Of Cartel Duration: A Cross-Sectional Study Of Modern Private International Cartels, Working Paper, West Lafayette.

Connor, J. (1998), The Global Citric Acid Conspiracy: Legal-Economic Lessons, Agribusiness, Vol. 14, 435-452.

Crooke, P., Froeb, L., Tschantz, L. und G.Werden (1999), The Effects of Assumed Demand Form on Simulated Post-Merger Equilibria, Review of Industrial Organization, Vol. 15, 205-217.

Czarnitzki; D. und A. Fier (2005), Zum Stand der empirischen Wirkungsanalyse der öffentlichen Innovations- und Forschungsförderung, Mannheim.

Czarnitzki, D., Fier, A., Hussinger, K. und G. Licht (2003): Einsatzmöglichkeiten neuer quantitativer Ansätze zur Wirkungsanalyse öffentlicher Forschungsförderung. Eine Grundlagenstudie, Endbericht für das Bundesministerium für Bildung und Forschung, Mannheim.

Deloitte (2007), The Deterrent Effect of Competition Enforcement by the OFT, London.

De Roos, N. (2006), Examining Models of Collusion: The Market for Lysine, International Journal of Industrial Organization, Vol. 24, 1083-1107.

Deutsche Gesellschaft für Evaluation (2002), Standards für Evaluation. Köln.

Don, H., Kemp, R. und J. van Sinderen (2008), Measuring the Economic Effects of Competition Law Enforcement, De Economist, Vol. 156, 341-348.

Duso, T., Gugler, K. und B. Yurtoglu (2006), How Effective is European Merger Control? WZB Discussion Paper SP II 2006-12, Berlin.

Eckbo, E. (1989), The Role of Stock Market Studies in Formulating Antitrust Policy Towards Horizontal Mergers, Quarterly Journal of Business and Economics, Vol. 28, 2238.

Epstein, R. und D. Rubinfeld (2001), Merger Simulation: A Simplified Approach with New Applications, Antitrust Law Journal, Vol. 69, 883-919.

Europäische Kommission (2005), Merger Remedies Study, Brüssel.

Evans, P. (2008), In Search for the Marginal Customer, FIPRA Study, Brüssel.

Federal Trade Commission (1999), A Study of the Commission's Divestiture Process, Prepared by the Staff of the Bureau of Competition of the Federal Trade Commission, Washington.

Feinberg, R. (1984), Strategic and Deterrent Pricing Responses to Antitrust Investigations, International Journal of Industrial Organization, Vol. 2, 75-84.

Frontier Economics (2006), The Impact of Regulation: A Pilot Study of the Incremental Costs and Benefits of Consumer and Competition Regulations, DTI Occasional Paper No. 7, London.

Gordon, F. und D. Squires (2008), The Deterrent Effect of UK Competition Enforcement, De Economist, Vol. 156, 411-432.

Harberger, A. (1954) Monopoly and Resource Allocation, American Economic Review Papers and Proceedings, Vol. 44, 77-87.

Harrington, J. (2006), How Do Cartels Operate?, Foundations and Trends in Microeconomics, Johns Hopkins University, Baltimore.

Høj, J., Jimenez, M., Maher, M., Nicoletti, G. und M. Wise (2007), Product Market Competition in the OECD Countries: Taking Stock and Moving Forward, OECD Economics Department Working Papers, No. 575, Paris. 
Hüschelrath, K. (2010), Detection of Anticompetitive Horizontal Mergers, forthcoming Journal of Competition Law and Economics.

Hüschelrath, K. (2009a), Competition Policy Analysis - An Integrated Approach, Heidelberg.

Hüschelrath, K. (2009b), Is it Worth all the Trouble? The Costs and Benefits of Antitrust Enforcement, forthcoming The Antitrust Bulletin.

Hüschelrath, K., Leheyda, N. und P. Beschorner (2009), Erfahrungen mit der Evaluation des Schweizer Kartellgesetzes, in dem vorliegenden Sammelband.

Jersey Competition Regulatory Authority (2008), Impacts of Competition Policy in the Bailiwick of Jersey, Saint Helier.

Konings, J., P. Van Cayseele, und F. Warzynski (2001), The Dynamics of Industrial Markups in Two Small Open Economies: Does National Competition Policy Matter? International Journal of Industrial Organization, Vol. 19, 841-859.

Kovacic, W. (2009), Assessing the Quality of Competition Policy: The Case of Horizontal Merger Enforcement, Competition Policy International, Vol. 5, 129-150.

Kovacic, W. (2006), Using Ex-post Evaluations to Improve the Performance of Competition Policy Authorities, Journal of Corporation Law, Vol. 31, 503-547.

Lear (2006), Ex-Post Review of Merger Control Decisions, Rome.

Levenstein, M. und V. Suslow (2004), What Determines Cartel Success? Working Paper, University of Michigan, Ann Arbor.

Neven, D. und H. Zenger (2008), Ex-post Evaluation of Enforcement: A Principal-Agent Perspective, De Economist, Vol. 156, 477-490.

Neven, D. und L.-H. Röller (2006), Consumer Surplus vs. Welfare Standard in a Political Economy Model of Merger Control, International Journal of Industrial Organization, Vol. 23, 829-848.

Nicholson, M. (2008), An Antitrust Law Index for Empirical Analysis of International Competition Policy, Journal of Competition Law \& Economics, Vol. 4, 1009-1029.

Nicholson, M. (2007), Quantifying Antitrust Regimes, Erasmus Law and Economics Review, Vol. 3, 41-62.

Niels, G. und R. van Dijk (2008), Competition Policy: What are the Costs and Benefits of Measuring its Costs and Benefits?, De Economist, Vol. 156, 349-364.

OECD (2008), Country Studies - Ukraine - Peer Review of Competition Law and Policy, Paris.

OECD (2005), Evaluation of the Actions and Resources of the Competition Authorities,

Policy Roundtables, Paris.

Office of Fair Trading (2008a), Positive Impact 07/08 - Consumer Benefits from Competition Enforcement, Merger Control, Market Studies and Market Investigation References, and Scam Busting, London.

Office of Fair Trading (2008b), Approach to Calculating Direct Benefits to Consumers, OFT 955, London.

Office of Fair Trading (2007a), Positive Impact 06/07. Consumer Benefits from Competition Enforcement, Merger Control, and Scam Busting, OFT 928, London.

Office of Fair Trading (2007b), Consumer Savings from Merger Control. Merger Simulation for Impact Estimation, OFT 917, London.

Office of Fair Trading (2005), Positive Impact: An Initial Evaluation of the Effect of the Competition Enforcement Work conducted by the Office of Fair Trading, OFT 837, London.

Oxera (2004), Costs and Benefits of Market Regulators, Part I: Conceptual Framework, Oxford.

Peters, C. (2006), Evaluating the Performance of Merger Simulations: Evidence from the U.S. Airline Industry, Journal o Law and Economics, Vol. 49, 627-649. 
PricewaterhouseCoopers (2005), Ex-post Evaluation of Mergers, Report for the UK Office of Fair Trading, Department of Trade and Industry and the Competition Commission, London.

Renckens, A. (2007), Welfare Standards, Substantive Tests, and Efficiency Considerations in Merger Policy: Defining the Efficiency Defence, Journal of Competition Law and Economics, Vol. 3, 149-179.

Sabbatini, P. (2008), Assessing the Impact of Antitrust Intervention by the Italian Competition Authority, De Economist, Vol. 156, 491-505.

Sanders, J. (2006), Handbuch der Evaluationsstandards - Die Standards des ,Joint Committee on Standards for Educational Evaluation', 3. Auflage, Wiesbaden.

Saurer, M. (2008), Zur Schweizerischen Wettbewerbspolitik - Schutz des Wettbewerbs oder der Wettbewerber?, Diskussionspapier, Avenir Suisse, Zürich.

Schinkel, M. und J. Tuinstra (2006), Imperfect Competition Law Enforcement, International Journal of Industrial Organization, forthcoming.

Seldeslachts, J., J. Clougherty und P. Barros (2006), Remedy for Now but Prevent for Tomorrow: The Deterrence Effect of Merger Policy Tools, Working Paper, WZB, Berlin.

Shughart, W. und R. Tollison (1998), Collusion, Profits, and Rational Antitrust, Antitrust Bulletin, Vol. 43, 365-374.

Slade, M. (2006), Merger Simulations of Unilateral Effects: What Can We Learn from the UK Brewing Industry?, Working Paper, Coventry.

Stigler, G. (1966), The Economic Effects of the Antitrust Laws, Journal of Law and Economics, Vol. 9, 225-258.

Stockmann, R. (2000), Evaluation in Deutschland, in: R. Stockmann (Hrsg.), Evaluationsforschung. Grundlagen und ausgewählte Forschungsfelder, Opladen, 11-40.

Suhling, S. und N. Ansorge (2008), Evaluation und Evaluationsforschung, Hannover.

Symeonidis, G. (2000), Price Competition and Market Structure: The Impact of Cartel Policy on Concentration in the UK, Journal of Industrial Economics, Vol. 48, 1-26.

Van Dijk, T. und F. Verboven (2005), Quantification of Damages, Working Paper, University of Antwerp, Antwerp.

Van Sinderen, J. und R. Kemp (2008), The Economic Effect of Competition Law Enforcement: The Case Of The Netherlands, De Economist, Vol. 156, 365-385.

Veljanovski, C. (2007a), Cartel Fines in Europe: Law, Practice and Deterrence, World Competition, Vol. 29, forthcoming.

Warzynski, F. (2001), Did Tough Antitrust Policy Lead to Lower Markups in the US Manufacturing Industry? Economics Letters, Vol. 70, 139-144.

Weinberg, M. (2008), The Price Effects of Horizontal Mergers, Journal of Competition Law and Economics, Vol. 4, 733-447.

Weinberg, M. (2006), An Evaluation of Mergers Simulations, Working Paper, Athens.

Weinberg, M. und D. Hosken (2009), Using Mergers to Test a Model of Oligopoly, FTC Working Paper, Washington D.C.

Werden, G. (2008), Assessing the Effects of Antitrust Enforcement in the United States, De Economist, Vol. 156, 433-451.

Werden, G. (1998), Demand Elasticities in Antitrust Analysis, Antitrust Law Journal, Vol. 66, 363-414.

Werden, G. (1996), A Robust Test for Consumer Welfare Enhancing Mergers among Sellers of Differentiated Products, Journal of Industrial Economics, Vol. 44, 409-413.

Werden, G. und L. Froeb (1994), The Effects of Mergers in Differentiated Products Industries: Logit Demand and Merger Policy, Journal of Law, Economics \& Organization, Vol. 10, 407-426. 